\title{
Dynamic interactions between double current-carrying nanowires immersed in a longitudinal magnetic field: Novel integro-surface energy-based models
}

\begin{abstract}
Transverse dynamic interactions between double current-carrying nanowires (CCNWs) immersed in a longitudinal magnetic field are of high interest. Using Biot-Savart and Lorentz laws, the approximate and exact magnetic forces exerted on the CCNWs are expressed. By employing Hamilton's principle, the equations of motion of magnetically affected CCNWs are obtained in the context of the surface elasticity theory of Gurtin-Murdoch. To this end, three approximate models as well as three exact versions of governing equations based on the Rayleigh, Timoshenko, and higher-order beam theories are developed. Via reproducing kernel particle method, the frequencies of the nanosystem are evaluated. With regard to the predicted results by the exact models, the application limits of the approximate models are displayed. Subsequently, the roles of the interwire distance, slenderness ratio, CCNWs' radius, electric current, and magnetic field strength on the fundamental frequency of the nanosystem are examined. For each of these explorations, the influences of the surface energy and shear deformation on the free dynamic response of the nanosystem are explained and discussed.
\end{abstract}

Keywords: Vibration; Double current-carrying nanowires; Magnetic field; Surface continuum-based beams; Reproducing kernel particle method (RKPM).

\section{Introduction}

Using piezoelectric materials, scientists have synthesized ensembles of vertically aligned nanowires (NWs) on a flat substrate as a nanogenerator (Qin et al. 2008; Wang and Song Preprint submitted to International Journal of Engineering Science

July 6, 2016 
2006; Wang et al. 2007). This advanced technology could release nanomachines from the bulk of batteries since allows harvesting mechanical energy from various environmental sources including ultrasonic waves, mechanical vibration or even blood flow. Further, the most apparent use of NWs is in electronics (Cui and Lieber 2001; Duan et al. 2001; Hahm and Lieber 2004). The NWs could be conductors, semiconductors or even superconductors depend on the environment's temperature and the NW's atomic structure. Due to their tiny dimensions, millions of millions transistors could be fitted on a single microprocessor and thereby, the computing speed would drastically increase. The current-carrying wires with lengths in the order of micrometers could be also exploited as precise magnetometers (Ando et al. 2009; Wickenden et al. 2003, 1997). The main functions of them is to map magnetic fields which have great potential applications in space physics, biomedicine, environmental sciences, oceanography, and transportation. For most of the above-mentioned applications, groups of current-carrying NWs (CCNWs) can be used. For optimal design of such nanosystems as well as magnetically affected ones, their free dynamic response and natural frequencies should be realized. To this end, vibration of magnetically affected double-nanowiresystem as electric current carrier is aimed to be investigated via more refined models. Such a scrutiny provides a crucial step towards better understanding vibrations of more complex nanosystems, like as vertically aligned CCNWs, in the presence of a magnetic field.

The surface of solids is a very thin layer whose atom arrangement and properties are different from those of the bulk (Dingreville and Cherkaoui 2005; Jing et al. 2006; Miller and Shenoy 2000). The classical continuum theory (CCT) cannot explain such a critical issue since it has been basically developed for macro-structures. By decreasing the sizes of a solid, the ratio of the surface area to the volume of the solid increases and the role of the surface effect in mechanical response of the structure becomes more important. To formulate such a vital effect, Gurtin and Murdoch (Gurtin and Murdoch 1975, 1976, 1978) proposed a novel theory to overcome the shortcomings of the CCT. In the newly developed model, the surface layer was introduced as a membrane of negligible thickness adhered to the underlying 
bulk without slippage. The constitutive relations of the surface layer are essentially different from those of the bulk. For an isotropic surface layer, the stresses are related to the strains through three parameters: residual surface stress, and two Lame's constants. In most of the cases, the equations of motion of the surface layer denote the nonclassical boundary conditions of the bulk. If the parameters associated with the surface layer tend to zero, the classical boundary conditions are retrieved and the size dependency is removed. Through solving the simultaneous equations of motion of the surface layer and the bulk accounting for the nonclassical conditions, the surface elasticity-based models are analyzed. To date, the surface elasticity theory of Gurtin and Murdoch (Gurtin and Murdoch 1975, 1976, 1978) has been widely applied to various problems of nanostructures such as statics (Khajeansari et al. 2012; Liu et al. 2012; Olsson and Park 2012; Park and Klein 2008), vibrations (Ansari et al. 2014a; Ansari and Sahmani 2011; Eltaher et al. 2012; Gheshlaghi and Hasheminejad 2011, 2012; Hashemi and Nazemnezhad 2013; Huang 2008; Malekzadeh and Shojaee 2013; Pishkenari et al. 2016; Wang and Feng 2010; Yan and Jiang 2011; Yan et al. 2012) and buckling (Ansari et al. 2014b; Li et al. 2011; Park 2012; Sahmani et al. 2016; Wang and Feng 2009; Zhang et al. 2012) of nanobeams/nanowires as well as free vibrations (Assadi 2013; Malekzadeh et al. 2014; Wang and Wang 2011) and buckling (Farajpour et al. 2013; Lu et al. 2011; Ravari and Shahidi 2013; Wang and Wang 2011; Zhang et al. 2012) of nanoplates. In all suggested models in the present work, the equations of motion are constructed in the framework of the surface elasticity theory of Gurtin-Murdoch.

Regarding individual magnetically affected CCNWs, any cause of deflection leads to exertion of extra transverse magnetic force on the nanowire. This force corresponds to the reduction of the lateral stiffness as well as the dynamic instability of the CCNWs. In the presence of a longitudinal magnetic field, free vibration and stability (Kiani 2014a), axial buckling (Kiani 2015a,b), forced vibration of individual CCNWs accounting for the nonlocality effect (Kiani 2014b) have been investigated. In the case of double CCNWs (DCCNWs), the vibration of each CCNW is also influenced by the magnetic field of the 
neighboring CCNW (Kiani 2015c,d). Thereby, the mechanical response of the constitutive CCNWs of the nanosystem would be entirely different from that of the magnetically affected individual CCNW.

By passing electric current through doubly parallel nanowires, a magnetic field would produce around each nanowire whose characteristics are displayed by the Biot-Savart's law. Such a magnetic field plus to the longitudinally applied one correspond to the magnetic force exerted on the deformed CCNWs. By employing the Lorentz force law, the exact expressions of the above-mentioned forces are derived in the form of integro-partial differential relations. For infinite lengths CCNWs, such expressions are reduced to those simple forms which are available in the literature (Kiani 2015c,d). Such simplified formulations represent approximate version of the magnetic forces. Investigation of the capabilities of the approximate models in predicting vibration behavior of the nanosystem based on the exact formulations is one of the main goals of the present work.

This paper deals with free transverse vibrations of and dynamic interactions between DCCNWs subjected to a longitudinal magnetic field. For this purpose, the exerted magnetic force on each CCNW is evaluated based on an approximate model and an exact one. In fact, the approximate model has been basically established based on the common assumption of infinite length of the nanowires. In the newly exact model, the length of the nanowires is appropriately incorporated into the dynamic magnetic forces evaluated on the basis of the Biot-Savart law. To study transverse vibrations of the magnetically affected CCNWs and their dynamic interactions, the equations of motion are obtained according to the Rayleigh beam theory (RBT), Timoshenko beam theory (TBT), and higher-order beam theory (HOBT). In all developed models, the surface energy effect of the nanosystem is incorporated into the formulations. Based on the above-mentioned models of beams and magnetic forces, six models are developed. The approximate governing equations based on the RBT, TBT, and HOBT in order represent four, eight, and eight coupled partial differential equations (PDEs); however, those obtained using exact version of the magnetic force 
are inherently four, eight, and eight integro-partial differential equations (IPDEs). Finding an analytical solution to these equations is a very cumbersome job. To overcome this difficulty, the reproducing kernel particle method (RKPM) is employed to predict the free vibration behavior of the nanosystem. This efficient meshless methodology was developed by Liu et al. (Liu et al. 1995a,b) and has been widely exploited for numerical analysis of engineering and physical problems (Chen et al. 1998; Cheng and Liew 2009; Ji-fa et al. 2005; Kiani 2010; Liew et al. 2002; Liu et al. 1997; Nianfei et al. 2009; Zhang et al. 2003). The obtained results by this method are verified with those of assumed mode method (AMM) in a particular case, and a reasonably good agreement is achieved. Thereafter, the effects of the slenderness ratio, interwire distance, CCNW's radius, magnetic field strength, and electric current on the fundamental frequency of the nanosystem are addressed. In the carried out studies, the roles of the surface energy and shear deformation on the obtained results are also explained in some detail. This work can be regarded as a pivotal step toward exploration of vibrations of forest of CCNWs which would have extensive applications in the upcoming nanoelectromechanical systems (NEMS).

\section{Definition of the nanomechanical problem}

A DCCNWs immersed in a longitudinal magnetic field is considered as shown in Fig. 1. The Cartesian coordinate systems $x_{i^{-}} y_{i^{-}} z_{i} ; i=1,2$ have been attached to one ends of the CCNWs such that the $x_{i}$ axis is coincident with the revolutionary axes of the $i$ th CCNW. The unit base vectors pertinent to these two coordinate systems are represented by $\mathbf{e}_{x_{i}}, \mathbf{e}_{y_{i}}$, $\mathbf{e}_{z_{i}}$. The length, interwire distance, NWs' radius, cross-sectional area, and moment inertia of the NWs in order are represented by $l_{b}, d, r_{0}, A_{b}$, and $I_{b}$. Each NW carries a constant electric current $I_{0}$ and is subjected to the initial tensile force of magnitude $T_{0}$ and a constant magnetic field $\mathbf{B}=B_{0} \mathbf{e}_{x_{i}}$. The transverse displacements of the $i$ th CCNW along the $y_{i}$ and $z_{i}$ axes are denoted by the $u_{y_{i}}^{[\cdot]}=u_{y_{i}}^{[\cdot]}\left(x_{i}, y_{i}, z_{i}, t\right)$ and $u_{z_{i}}^{[\cdot]}=u_{z_{i}}^{[\cdot]}\left(x_{i}, y_{i}, z_{i}, t\right) ; i=1,2$ where [.] stands for the theory used for analyzing the problem. In the present paper, the parameters 
with the superscripts $R, T$, and $H$ are associated with the NWs modeled on the basis RBT, TBT, and HOBT, respectively.

In the following part, the magnetic forces on the laterally deformed CCNWs in the presence of a longitudinal magnetic field are evaluated in some details.

\section{The magnetic forces exerted on the CCNWs}

In the framework of small deformations, it can be readily shown that the electric currents pass through the laterally deformed NWs in vectorial form are stated by:

$$
\mathbf{I}_{i}=I_{0}\left(\mathbf{e}_{x_{i}}+\frac{\partial u_{y_{i}}^{[\cdot]}}{\partial x_{i}} \mathbf{e}_{y_{i}}+\frac{\partial u_{z_{i}}^{[.]}}{\partial x_{i}} \mathbf{e}_{z_{i}}\right)
$$

Each deformed NW is affected by the externally exerted magnetic field as well as that produced by its adjacent deformed CCNW. By employing the Biot-Savart law, the resultant magnetic fields at the vicinity of the laterally deformed CCNWs are expressed as:

$$
\begin{aligned}
& \mathbf{B}_{1}=B_{0} \mathbf{e}_{x_{1}}+\frac{\mu_{0}}{4 \pi} \int_{0}^{l_{b}} \frac{\mathbf{I}_{2} \times \mathbf{r}_{2}}{\left\|\mathbf{r}_{2}\right\|^{3}} \mathrm{~d} s_{1}, \\
& \mathbf{B}_{2}=B_{0} \mathbf{e}_{x_{2}}+\frac{\mu_{0}}{4 \pi} \int_{0}^{l_{b}} \frac{\mathbf{I}_{1} \times \mathbf{r}_{1}}{\left\|\mathbf{r}_{1}\right\|^{3}} \mathrm{~d} s_{2},
\end{aligned}
$$

where $\mu_{0}=4 \pi \times 10^{-7} \frac{\mathrm{Tm}}{\mathrm{A}}$ is the permeability of free space, $\mathrm{d} s_{1}$ and $\mathrm{d} s_{2}$ in order are the infinitesimal lengths of the first and second NWs, and $\|$.$\| represents the norm symbol. The$ position vectors $\mathbf{r}_{i} ; i=1,2$ are defined by:

$$
\mathbf{r}_{1}=-\mathbf{r}_{2}=\left(x_{2}-x_{1}\right) \mathbf{e}_{x_{1}}+\left(d+\Delta u_{y}^{[\cdot]}\right) \mathbf{e}_{y_{1}}+\Delta u_{z}^{[\cdot]} \mathbf{e}_{z_{1}}
$$

in which $\Delta u_{y}^{[.]}=u_{y_{2}}^{[.]}-u_{y_{1}}^{[.]}$and $\Delta u_{z}^{[.]}=u_{z_{2}}^{[\cdot]}-u_{z_{1}}^{[.]}$. Based on the Lorentz's law, the exerted magnetic forces per unit length of the $i$ th $\mathrm{CCNW}$ are evaluated by: $\mathbf{f}_{m_{i}}=\mathbf{I}_{i} \times \mathbf{B}_{i} ; i=1,2$. 


\subsection{An approximation formulation}

For fairly lengthy NWs (i.e., $-\infty<l_{b}<\infty$ ), the components of the magnetic fields at the location of deformed CCNWs are obtained by using Eqs. (2a) and (2b):

$$
\begin{aligned}
\mathbf{B}_{1}= & \left(B_{0}-\frac{\mu_{0} I_{0}}{2 \pi} \frac{\Delta w^{[\cdot]} \frac{\partial v_{2}^{[\cdot]}}{\partial x_{2}}-\left(d+\Delta v^{[\cdot]}\right) \frac{\partial w_{2}^{[\cdot]}}{\partial x_{2}}}{\left(d+\Delta v^{[\cdot]}\right)^{2}+\left(\Delta w^{[\cdot]}\right)^{2}}\right) \mathbf{e}_{x_{1}} \\
& +\left(\frac{\mu_{0} I_{0}}{2 \pi} \frac{\Delta w^{[\cdot]}}{\left(d+\Delta v^{[\cdot]}\right)^{2}+\left(\Delta w^{[\cdot])^{2}}\right.}\right) \mathbf{e}_{y_{1}}-\left(\frac{\mu_{0} I_{0}}{2 \pi} \frac{d+\Delta v^{[\cdot]}}{\left(d+\Delta v^{[\cdot]}\right)^{2}+\left(\Delta w^{[\cdot]}\right)^{2}}\right) \mathbf{e}_{z_{1}}, \\
\mathbf{B}_{2}= & \left(B_{0}+\frac{\mu_{0} I_{0}}{2 \pi} \frac{\Delta w^{[\cdot]} \frac{\partial v_{1}^{[\cdot]}}{\partial x_{1}}-\left(d+\Delta v^{[\cdot]}\right) \frac{\partial w_{1}^{[\cdot]}}{\partial x_{1}}}{\left(d+\Delta v^{[\cdot]}\right)^{2}+\left(\Delta w^{[\cdot]}\right)^{2}}\right) \mathbf{e}_{x_{2}} \\
& -\left(\frac{\mu_{0} I_{0}}{2 \pi} \frac{\Delta w^{[\cdot]}}{\left(d+\Delta v^{[\cdot]}\right)^{2}+\left(\Delta w^{[\cdot]}\right)^{2}}\right) \mathbf{e}_{y_{2}}+\left(\frac{\mu_{0} I_{0}}{2 \pi} \frac{d+\Delta v^{[\cdot]}}{\left(d+\Delta v^{[\cdot]}\right)^{2}+\left(\Delta w^{[\cdot]}\right)^{2}}\right) \mathbf{e}_{z_{2}} .
\end{aligned}
$$

Now, consider the following relations:

$$
\begin{gathered}
\frac{d+\Delta v^{[\cdot]}}{\left(d+\Delta v^{[\cdot]}\right)^{2}+\left(\Delta w^{[\cdot]}\right)^{2}} \approx \frac{1}{d}\left(1-\frac{\Delta v^{[\cdot]}}{d}+\left(\frac{\Delta v^{[\cdot]}}{d}\right)^{2}\right), \\
\frac{\Delta w^{[\cdot]}}{\left(d+\Delta v^{[\cdot]}\right)^{2}+\left(\Delta w^{[\cdot]}\right)^{2}} \approx \frac{\Delta w^{[\cdot]}}{d^{2}}\left(1-\left(\frac{\Delta w^{[\cdot]}}{d}\right)^{2}\right) .
\end{gathered}
$$

By introducing Eqs. (5a) and (5b) to Eqs. (4a) and (4b) and by implementing the Lorentz's formulas, the applied magnetic forces on the deformed CCNWs are obtained as:

$$
\mathbf{f}_{m_{i}}^{[\cdot]}=f_{m_{x i}}^{[\cdot]} \mathbf{e}_{x_{i}}+f_{m_{y i}}^{[\cdot]} \mathbf{e}_{y_{i}}+f_{m_{z i}}^{[\cdot]} \mathbf{e}_{z_{i}} ; i=1,2
$$

where

$$
\begin{aligned}
& f_{m_{x 1}}^{[\cdot]} \approx \frac{\mu_{0} I_{0}^{2}}{2 \pi d}\left[\frac{\partial v_{1}^{[\cdot]}}{\partial x_{1}}\left(1+\frac{\Delta v^{[\cdot]}}{d}+\left(\frac{\Delta v^{[\cdot]}}{d}\right)^{2}\right)-\frac{\partial w_{2}^{[\cdot]}}{\partial x_{2}}\left(\frac{\Delta w^{[\cdot]}}{d}\right)\left(1-\left(\frac{\Delta w^{[\cdot]}}{d}\right)^{2}\right)\right], \\
& f_{m_{y 1}}^{[.]} \approx B_{0} I_{0} \frac{\partial w_{1}^{[\cdot]}}{\partial x_{1}}-\frac{\mu_{0} I_{0}^{2}}{2 \pi d}\left[\begin{array}{l}
\frac{\partial v_{1}^{[.]}}{\partial x_{1}} \frac{\partial w_{1}^{[\cdot]}}{\partial x_{1}}\left(\frac{\Delta w^{[\cdot]}}{d}\right)\left(1-\left(\frac{\Delta w^{[\cdot]}}{d}\right)^{2}\right)+ \\
\left(1+\left(\frac{\partial w_{1}^{[\cdot]}}{\partial x_{1}}\right)^{2}\right)\left(1+\frac{\Delta v^{[\cdot]}}{d}+\left(\frac{\Delta v^{[\cdot]}}{d}\right)^{2}\right)
\end{array}\right],
\end{aligned}
$$




$$
f_{m_{z 1}}^{[.]} \approx-B_{0} I_{0} \frac{\partial v_{1}^{[\cdot]}}{\partial x_{1}}+\frac{\mu_{0} I_{0}^{2}}{2 \pi d}\left[\begin{array}{l}
\frac{\partial v_{1}^{[.]}}{\partial x_{1}} \frac{\partial w_{1}^{[.]}}{\partial x_{1}}\left(1+\frac{\Delta v^{[\cdot]}}{d}+\left(\frac{\Delta v^{[\cdot]}}{d}\right)^{2}\right)+ \\
\left(1+\left(\frac{\partial v_{1}^{[.]}}{\partial x_{1}}\right)^{2}\right)\left(\frac{\Delta w^{[\cdot]}}{d}\right)\left(1-\left(\frac{\Delta w^{[\cdot]}}{d}\right)^{2}\right)
\end{array}\right]
$$

and

$$
\begin{aligned}
& f_{m_{x 2}}^{[\cdot]} \approx \frac{\mu_{0} I_{0}^{2}}{2 \pi d}\left[\frac{\partial v_{2}^{[\cdot]}}{\partial x_{2}}\left(1-\frac{\Delta v^{[\cdot]}}{d}+\left(\frac{\Delta v^{[\cdot]}}{d}\right)^{2}\right)+\frac{\partial w_{2}^{[\cdot]}}{\partial x_{2}}\left(\frac{\Delta w^{[\cdot]}}{d}\right)\left(1-\left(\frac{\Delta w^{[\cdot]}}{d}\right)^{2}\right)\right], \\
& f_{m_{y 2}}^{[\cdot]} \approx B_{0} I_{0} \frac{\partial w_{2}^{[\cdot]}}{\partial x_{2}}+\frac{\mu_{0} I_{0}^{2}}{2 \pi d}\left[\begin{array}{l}
\frac{\partial v_{2}^{[\cdot]}}{\partial x_{2}} \frac{\partial w_{2}^{[\cdot]}}{\partial x_{2}}\left(\frac{\Delta w^{[\cdot]}}{d}\right)\left(1-\left(\frac{\Delta w^{[\cdot]}}{d}\right)^{2}\right)- \\
\left(1+\left(\frac{\partial w_{2}^{[\cdot]}}{\partial x_{2}}\right)^{2}\right)\left(1-\frac{\Delta v^{[\cdot]}}{d}+\left(\frac{\Delta v^{[\cdot]}}{d}\right)^{2}\right)
\end{array}\right] \\
& f_{m_{z 2}}^{[\cdot]} \approx-B_{0} I_{0} \frac{\partial v_{2}^{[.]}}{\partial x_{2}}+\frac{\mu_{0} I_{0}^{2}}{2 \pi d}\left[\begin{array}{l}
\frac{\partial v_{2}^{[.]}}{\partial x_{2}} \frac{\partial w_{2}^{[\cdot]}}{\partial x_{2}}\left(1-\frac{\Delta v^{[\cdot]}}{d}+\left(\frac{\Delta v^{[\cdot]}}{d}\right)^{2}\right)- \\
\left(1+\left(\frac{\partial v_{2}^{[.]}}{\partial x_{2}}\right)^{2}\right)\left(\frac{\Delta w^{[\cdot]}}{d}\right)\left(1-\left(\frac{\Delta w^{[\cdot]}}{d}\right)^{2}\right)
\end{array}\right]
\end{aligned}
$$

By neglecting the products of partial derivatives of transverse displacements as well as the square of ratio of relative displacements to the interwire distance, the magnetic forces exerted on the deformed CCNWs reduce to the following linear expressions:

$$
\begin{aligned}
\mathbf{f}_{m_{1}}^{[\cdot]}= & \frac{\mu_{0} I_{0}^{2}}{2 \pi d} \frac{\partial v_{1}^{[\cdot]}}{\partial x_{1}} \mathbf{e}_{x_{1}}+\left(B_{0} I_{0} \frac{\partial w_{1}^{[\cdot]}}{\partial x_{1}}+\frac{\mu_{0} I_{0}^{2}}{2 \pi d}\left(1-\frac{\Delta v^{[\cdot]}}{d}\right)\right) \mathbf{e}_{y_{1}} \\
& +\left(-B_{0} I_{0} \frac{\partial v_{1}^{[\cdot]}}{\partial x_{1}}+\frac{\mu_{0} I_{0}^{2}}{2 \pi d}\left(\frac{\Delta w^{[\cdot]}}{d}\right)\right) \mathbf{e}_{z_{1}}, \\
\mathbf{f}_{m_{2}}^{[\cdot]}= & \frac{\mu_{0} I_{0}^{2}}{2 \pi d} \frac{\partial v_{2}^{[\cdot]}}{\partial x_{2}} \mathbf{e}_{x_{2}}+\left(B_{0} I_{0} \frac{\partial w_{2}^{[\cdot]}}{\partial x_{2}}-\frac{\mu_{0} I_{0}^{2}}{2 \pi d}\left(1-\frac{\Delta v^{[\cdot]}}{d}\right)\right) \mathbf{e}_{y_{2}} \\
& +\left(-B_{0} I_{0} \frac{\partial v_{2}^{[\cdot]}}{\partial x_{2}}-\frac{\mu_{0} I_{0}^{2}}{2 \pi d}\left(\frac{\triangle w^{[\cdot]}}{d}\right)\right) \mathbf{e}_{z_{2}} .
\end{aligned}
$$


Since transverse vibrations of the nanosystem is of interest, the longitudinal component of the magnetic forces as well as the statically applied forces on the CCNWs are excluded from Eqs. (9a) and (9b). Therefore, the transverse dynamic magnetic forces on the CCNWs take the following form:

$$
\begin{aligned}
& \mathbf{f}_{m_{1}}^{[\cdot]}=\left(B_{0} I_{0} \frac{\partial w_{1}^{[\cdot]}}{\partial x_{1}}-\frac{\mu_{0} I_{0}^{2}}{2 \pi d^{2}}\left(v_{2}^{[\cdot]}-v_{1}^{[\cdot]}\right)\right) \mathbf{e}_{y_{1}}+\left(-B_{0} I_{0} \frac{\partial v_{1}^{[\cdot]}}{\partial x_{1}}+\frac{\mu_{0} I_{0}^{2}}{2 \pi d^{2}}\left(w_{2}^{[\cdot]}-w_{1}^{[\cdot]}\right)\right) \mathbf{e}_{z_{1}},(10 \mathrm{a}) \\
& \mathbf{f}_{m_{2}}^{[\cdot]}=\left(B_{0} I_{0} \frac{\partial w_{2}^{[\cdot]}}{\partial x_{2}}+\frac{\mu_{0} I_{0}^{2}}{2 \pi d^{2}}\left(v_{2}^{[\cdot]}-v_{1}^{[\cdot]}\right)\right) \mathbf{e}_{y_{2}}-\left(B_{0} I_{0} \frac{\partial v_{2}^{[\cdot]}}{\partial x_{2}}+\frac{\mu_{0} I_{0}^{2}}{2 \pi d^{2}}\left(w_{2}^{[\cdot]}-w_{1}^{[\cdot]}\right)\right) \mathbf{e}_{z_{2}} \cdot(10 \mathrm{~b})
\end{aligned}
$$

\subsection{An exact formulation}

By substituting Eqs. (1) and (3) into Eqs. (2a) and (2b), the magnetic fields at the vicinity of the CCNWs are evaluated exactly as follows:

$$
\begin{aligned}
\mathbf{B}_{1}= & \left(B_{0}-\frac{\mu_{0} I_{0}}{4 \pi} \int_{0}^{l_{b}} \frac{\Delta w^{[\cdot]} \frac{\partial v_{2}^{[\cdot]}}{\partial x_{2}}-\left(d+\Delta v^{[\cdot]}\right) \frac{\partial w_{2}^{[\cdot]}}{\partial x_{2}}}{\left(\left(x_{2}-x_{1}\right)^{2}+\left(d+\Delta v^{[\cdot]}\right)^{2}+\left(\Delta w^{[\cdot]}\right)^{2}\right)^{\frac{3}{2}}} \mathrm{~d} x_{2}\right) \mathbf{e}_{x_{1}}- \\
& \left(\frac{\mu_{0} I_{0}}{4 \pi} \int_{0}^{l_{b}} \frac{\left(x_{2}-x_{1}\right) \frac{\partial w_{2}^{[\cdot]}}{\partial x_{2}}-\Delta w^{[\cdot]}}{\left(\left(x_{2}-x_{1}\right)^{2}+\left(d-\Delta v^{[\cdot]}\right)^{2}+\left(\Delta w^{[\cdot]}\right)^{2}\right)^{\frac{3}{2}}} \mathrm{~d} x_{2}\right) \mathbf{e}_{y_{1}}- \\
& \left(\frac{\mu_{0} I_{0}}{4 \pi} \int_{0}^{l_{b}} \frac{\left(d+\Delta v^{[\cdot]}\right)-\left(x_{2}-x_{1}\right) \frac{\partial v_{2}^{[\cdot]}}{\partial x_{2}}}{\left(\left(x_{2}-x_{1}\right)^{2}+\left(d+\Delta v^{[\cdot]}\right)^{2}+\left(\Delta w^{[\cdot]}\right)^{2}\right)^{\frac{3}{2}}} \mathrm{~d} x_{1}\right) \mathbf{e}_{z_{1}},
\end{aligned}
$$

and

$$
\begin{aligned}
\mathbf{B}_{2}= & \left(B_{0}+\frac{\mu_{0} I_{0}}{4 \pi} \int_{0}^{l_{b}} \frac{\Delta w^{[\cdot]} \frac{\partial v_{1}^{[\cdot]}}{\partial x_{1}}-\left(d+\Delta v^{[\cdot]}\right) \frac{\partial w_{1}^{[\cdot]}}{\partial x_{1}}}{\left(\left(x_{2}-x_{1}\right)^{2}+\left(d+\Delta v^{[\cdot]}\right)^{2}+\left(\Delta w^{[\cdot]}\right)^{2}\right)^{\frac{3}{2}}} \mathrm{~d} x_{1}\right) \mathbf{e}_{x_{2}}+ \\
& \left(\frac{\mu_{0} I_{0}}{4 \pi} \int_{0}^{l_{b}} \frac{\left(x_{2}-x_{1}\right) \frac{\partial w_{1}^{[\cdot]}}{\partial x_{1}}-\Delta w^{[\cdot]}}{\left(\left(x_{2}-x_{1}\right)^{2}+\left(d+\Delta v^{[\cdot]}\right)^{2}+\left(\Delta w^{[\cdot]}\right)^{2}\right)^{\frac{3}{2}}} \mathrm{~d} x_{1}\right) \mathbf{e}_{y_{2}}+ \\
& \left(\frac{\mu_{0} I_{0}}{4 \pi} \int_{0}^{l_{b}} \frac{\left(d+\Delta v^{[\cdot]}\right)-\left(x_{2}-x_{1}\right) \frac{\partial v_{1}^{[\cdot]}}{\partial x_{1}}}{\left(\left(x_{2}-x_{1}\right)^{2}+\left(d+\Delta v^{[\cdot]}\right)^{2}+\left(\Delta w^{[\cdot]}\right)^{2}\right)^{\frac{3}{2}}} \mathrm{~d} x_{1}\right) \mathbf{e}_{z_{2}} .
\end{aligned}
$$


By exploiting the Lorentz's formula, the components of the magnetic forces on the CCNWs immersed in a magnetic field are calculated as:

$$
\begin{aligned}
& f_{m_{x 1}}^{[.]}=-\frac{\mu_{0} I_{0}^{2}}{4 \pi} \int_{0}^{l_{b}} \frac{\left(d+\Delta v^{[\cdot]}\right) \frac{\partial v_{1}^{[\cdot]}}{\partial x_{1}}+\left(x_{2}-x_{1}\right)\left(\frac{\partial v_{1}^{[\cdot]}}{\partial x_{1}} \frac{\partial v_{2}^{[\cdot]}}{\partial x_{2}}+\frac{\partial w_{1}^{[\cdot]}}{\partial x_{1}} \frac{\partial w_{2}^{[\cdot]}}{\partial x_{2}}\right)+\Delta w^{[\cdot]} \frac{\partial w_{1}^{[\cdot]}}{\partial x_{1}}}{\left(\left(x_{2}-x_{1}\right)^{2}+\left(d+\Delta v^{[\cdot]}\right)^{2}+\left(\Delta w^{[\cdot]}\right)^{2}\right)^{\frac{3}{2}}} \mathrm{~d} x_{2},(13 \mathrm{a}) \\
& f_{m_{y 1}}^{[.]}=B_{0} I_{0} \frac{\partial w_{1}^{[\cdot]}}{\partial x_{1}}-\frac{\mu_{0} I_{0}^{2}}{4 \pi} \int_{0}^{l_{b}} \frac{\Delta w^{[\cdot]} \frac{\partial w_{1}^{[\cdot]}}{\partial x_{1}} \frac{\partial v_{2}^{[\cdot]}}{\partial x_{2}}+\left(d+\Delta v^{[\cdot]}\right)\left(1+\frac{\partial w_{1}^{[.]}}{\partial x_{1}} \frac{\partial w_{2}^{[\cdot]}}{\partial x_{2}}\right)+\left(x_{2}-x_{1}\right) \frac{\partial v_{2}^{[\cdot]}}{\partial x_{2}}}{\left(\left(x_{2}-x_{1}\right)^{2}+\left(d+\Delta v^{[\cdot]}\right)^{2}+\left(\Delta w^{[\cdot]}\right)^{2}\right)^{\frac{3}{2}}} \mathrm{~d} x_{2},(13) \\
& f_{m_{z 1}}^{[\cdot]}=-B_{0} I_{0} \frac{\partial v_{1}^{[\cdot]}}{\partial x_{1}}-\frac{\mu_{0} I_{0}^{2}}{4 \pi} \int_{0}^{l_{b}} \frac{\left(x_{2}-x_{1}\right) \frac{\partial w_{2}^{[\cdot]}}{\partial x_{2}}+\Delta w^{[\cdot]}\left(1+\frac{\partial v_{1}^{[.]}}{\partial x_{1}} \frac{\partial v_{2}^{[\cdot]}}{\partial x_{2}}\right)+\left(d+\Delta v^{[\cdot]}\right) \frac{\partial w_{1}^{[\cdot]}}{\partial x_{1}} \frac{\partial v_{2}^{[\cdot]}}{\partial x_{2}}}{\left(\left(x_{2}-x_{1}\right)^{2}+\left(d+\Delta v^{[\cdot]}\right)^{2}+\left(\Delta w^{[\cdot]}\right)^{2}\right)^{\frac{3}{2}}} \mathrm{~d} x_{2},(13 \mathrm{c}) \\
& f_{m_{x 2}}^{[\cdot]}=\frac{\mu_{0} I_{0}^{2}}{4 \pi} \int_{0}^{l_{b}} \frac{\left(d+\Delta v^{[\cdot]}\right) \frac{\partial v_{2}^{[\cdot]}}{\partial x_{2}}-\left(x_{2}-x_{1}\right)\left(\frac{\partial v_{1}^{[\cdot]}}{\partial x_{1}} \frac{\partial v_{2}^{[\cdot]}}{\partial x_{2}}+\frac{\partial w_{1}^{[\cdot]}}{\partial x_{1}} \frac{\partial w_{2}^{[\cdot]}}{\partial x_{2}}\right)-\Delta w^{[\cdot]} \frac{\partial w_{2}^{[\cdot]}}{\partial x_{2}}}{\left(\left(x_{2}-x_{1}\right)^{2}+\left(d+\Delta v^{[\cdot]}\right)^{2}+\left(\Delta w^{[\cdot]}\right)^{2}\right)^{\frac{3}{2}}} \mathrm{~d} x_{1}, \\
& f_{m_{y 2}}^{[\cdot]}=B_{0} I_{0} \frac{\partial w_{2}^{[\cdot]}}{\partial x_{2}}+\frac{\mu_{0} I_{0}^{2}}{4 \pi} \int_{0}^{l_{b}} \frac{\Delta w^{[\cdot]} \frac{\partial v_{1}^{[\cdot]}}{\partial x_{1}} \frac{\partial w_{2}^{[\cdot]}}{\partial x_{2}}-\left(d+\Delta v^{[\cdot]}\right)\left(1+\frac{\partial w_{1}^{[\cdot]}}{\partial x_{1}} \frac{\partial w_{2}^{[\cdot]}}{\partial x_{2}}\right)+\left(x_{2}-x_{1}\right) \frac{\partial v_{1}^{[\cdot]}}{\partial x_{1}}}{\left(\left(x_{2}-x_{1}\right)^{2}+\left(d+\Delta v^{[\cdot]}\right)^{2}+\left(\Delta w^{[\cdot]}\right)^{2}\right)^{\frac{3}{2}}} \mathrm{~d} x_{1},(13 \mathrm{e}) \\
& f_{m_{z 2}}^{[\cdot]}=-B_{0} I_{0} \frac{\partial v_{2}^{[\cdot]}}{\partial x_{2}}+\frac{\mu_{0} I_{0}^{2}}{4 \pi} \int_{0}^{l_{b}} \frac{\left(x_{2}-x_{1}\right) \frac{\partial w_{1}^{[\cdot]}}{\partial x_{1}}-\Delta w^{[\cdot]}\left(1+\frac{\partial v_{1}^{[\cdot]}}{\partial x_{1}} \frac{\partial v_{2}^{[\cdot]}}{\partial x_{2}}\right)+\left(d+\Delta v^{[\cdot]}\right) \frac{\partial w_{1}^{[\cdot]}}{\partial x_{1}} \frac{\partial v_{2}^{[\cdot]}}{\partial x_{2}}}{\left(\left(x_{2}-x_{1}\right)^{2}+\left(d+\Delta v^{[\cdot]}\right)^{2}+\left(\Delta w^{[\cdot]}\right)^{2}\right)^{\frac{3}{2}}} \mathrm{~d} x_{1} \cdot(13
\end{aligned}
$$

By assuming $\Delta v \ll d$ and $\Delta w \ll d$, neglecting the products of the first derivatives of displacements, and excluding the static terms, the dynamically exerted magnetic force on 
the CCNWs are obtained as:

$$
\begin{aligned}
& \mathbf{f}_{m_{1}}^{[\cdot]}=\left(\frac{\mu_{0} I_{0}^{2}}{4 \pi} \int_{0}^{l_{b}} \frac{d}{\left(\left(x_{2}-x_{1}\right)^{2}+d^{2}\right)^{\frac{3}{2}}} \frac{\partial v_{1}^{[\cdot]}}{\partial x_{1}} \mathrm{~d} x_{2}\right) \mathbf{e}_{x_{1}}+ \\
& \left(B_{0} I_{0} \frac{\partial w_{1}^{[\cdot]}}{\partial x_{1}}-\frac{\mu_{0} I_{0}^{2}}{4 \pi} \int_{0}^{l_{b}} \frac{\left(x_{2}-x_{1}\right) \frac{\partial v_{2}^{[\cdot]}}{\partial x_{2}}-\Delta v^{[\cdot]}}{\left(\left(x_{2}-x_{1}\right)^{2}+d^{2}\right)^{\frac{3}{2}}} \mathrm{~d} x_{2}\right) \mathbf{e}_{y_{1}} \\
& +\left(-B_{0} I_{0} \frac{\partial v_{1}^{[\cdot]}}{\partial x_{1}}-\frac{\mu_{0} I_{0}^{2}}{4 \pi} \int_{0}^{l_{b}} \frac{\left(x_{2}-x_{1}\right) \frac{\partial w_{2}^{[\cdot]}}{\partial x_{2}}-\Delta w^{[\cdot]}}{\left(\left(x_{2}-x_{1}\right)^{2}+d^{2}\right)^{\frac{3}{2}}} \mathrm{~d} x_{2}\right) \mathbf{e}_{z_{1}}, \\
& \mathbf{f}_{m_{2}}^{[\cdot]}=\left(\frac{\mu_{0} I_{0}^{2}}{4 \pi} \int_{0}^{l_{b}} \frac{d}{\left(\left(x_{2}-x_{1}\right)^{2}+d^{2}\right)^{\frac{3}{2}}} \frac{\partial v_{2}^{[\cdot]}}{\partial x_{2}} \mathrm{~d} x_{1}\right) \mathbf{e}_{x_{2}}+ \\
& \left(B_{0} I_{0} \frac{\partial w_{2}^{[\cdot]}}{\partial x_{2}}+\frac{\mu_{0} I_{0}^{2}}{4 \pi} \int_{0}^{l_{b}} \frac{\left(x_{2}-x_{1}\right) \frac{\partial v_{1}^{[\cdot]}}{\partial x_{1}}-\Delta v^{[\cdot]}}{\left(\left(x_{2}-x_{1}\right)^{2}+d^{2}\right)^{\frac{3}{2}}} \mathrm{~d} x_{1}\right) \mathbf{e}_{y_{2}} \\
& +\left(-B_{0} I_{0} \frac{\partial v_{2}^{[\cdot]}}{\partial x_{2}}+\frac{\mu_{0} I_{0}^{2}}{4 \pi} \int_{0}^{l_{b}} \frac{\left(x_{2}-x_{1}\right) \frac{\partial w_{1}^{[\cdot]}}{\partial x_{1}}-\Delta w^{[\cdot]}}{\left(\left(x_{2}-x_{1}\right)^{2}+d^{2}\right)^{\frac{3}{2}}} \mathrm{~d} x_{1}\right) \mathbf{e}_{z_{2}} .
\end{aligned}
$$

\section{Development of surface energy-based formulations via RBT}

\subsection{Constitutive relations of the bulk and the surface layer}

By employing the RBT, the displacements of both surface layer and bulk of the constitutive CCNWs of the nanosystem are stated by:

$$
\begin{aligned}
& u_{x_{i}}^{R}\left(x_{i}, y_{i}, z_{i}, t\right)=-\left(z_{i} \frac{\partial w_{i}^{R}\left(x_{i}, t\right)}{\partial x_{i}}+y_{i} \frac{\partial v_{i}^{R}\left(x_{i}, t\right)}{\partial x_{i}}\right), \\
& u_{y_{i}}^{R}\left(x_{i}, y_{i}, z_{i}, t\right)=v_{i}^{R}\left(x_{i}, t\right), \quad u_{z_{i}}^{R}\left(x_{i}, y_{i}, z_{i}, t\right)=w_{i}^{R}\left(x_{i}, t\right) .
\end{aligned}
$$

For the small deformation regime, the only nonzero component of the strain field of both surface and bulk reads:

$$
\epsilon_{x x_{i}}^{R}=-\left(z_{i} \frac{\partial^{2} w_{i}^{R}\left(x_{i}, t\right)}{\partial x_{i}^{2}}+y_{i} \frac{\partial^{2} v_{i}^{R}\left(x_{i}, t\right)}{\partial x_{i}^{2}}\right) .
$$


In the context of theory of elasticity of Gurtin-Murdoch Gurtin and Murdoch $(1975,1976$, 1978), the stress-strains of the surface layer are given by:

$$
\begin{gathered}
\tau_{\alpha \beta}=\tau_{0} \delta_{\alpha \beta}+\tau_{0} \frac{\partial u_{\alpha}}{\partial \beta}+2\left(\mu_{0}-\tau_{0}\right) \epsilon_{\alpha \beta}+\left(\lambda_{0}+\tau_{0}\right) \epsilon_{\gamma \gamma} \delta_{\alpha \beta} ; \alpha, \beta, \gamma=x, y, z, \\
\tau_{x \alpha}=\tau_{0} \frac{\partial u_{\alpha}}{\partial x} ; \alpha=y, z,
\end{gathered}
$$

where $\tau_{\alpha \beta}$ and $\epsilon_{\alpha \beta}$ are the surface stresses and strains, respectively, $\tau_{0}$ is the residual surface stress under unconstrained conditions, $\lambda_{0}$ and $\mu_{0}$ are the Lame's constants of the surface layer, and $\delta_{\alpha \beta}$ is the Kronecker delta tensor. In view of the given displacements in Eq. (15), the only nonzero stress components of the surface layer of the $i$ th CCNW on the basis of the RBT are as:

$$
\begin{aligned}
\tau_{x x_{i}}^{R} & =\tau_{0}+\left(\lambda_{0}+2 \mu_{0}\right) \frac{\partial u_{x_{i}}^{R}}{\partial x_{i}}=\tau_{0}-\left(\lambda_{0}+2 \mu_{0}\right)\left(z_{i} \frac{\partial^{2} w_{i}^{R}}{\partial x_{i}^{2}}+y_{i} \frac{\partial^{2} v_{i}^{R}}{\partial x_{i}^{2}}\right), \\
\tau_{x y_{i}}^{R} & =n_{y_{i}} \tau_{0} \frac{\partial v_{i}^{R}}{\partial x_{i}}, \quad \tau_{x z_{i}}^{R}=n_{z_{i}} \tau_{0} \frac{\partial w_{i}^{R}}{\partial x_{i}}
\end{aligned}
$$

where $\tau_{x x_{i}}^{R}$ is the longitudinal normal stress of the surface layer, $\tau_{x y_{i}}^{R}$ and $\tau_{x z_{i}}^{R}$ are the shear stresses, and $n_{y_{i}}$ and $n_{y_{i}}$ are the components of the unit outward normal vector of the surface layer of the $i$ th CCNW.

Now, it is assumed that the stress components $\sigma_{y y_{i}}^{R}$ and $\sigma_{z z_{i}}^{R}$ within the bulk vary linearly between their corresponding surface values. Thereby, the longitudinal stress within the bulk of the $i$ th CCNW based on the generalized Hook's law reads:

$$
\begin{aligned}
\sigma_{x x_{i}}^{R}= & -z_{i}\left(\left(E_{b}-\frac{2 \tau_{0} \nu_{b}}{D_{0}}\right) \frac{\partial^{2} w_{i}^{R}}{\partial x_{i}^{2}}+\frac{2 \rho_{0} \nu_{b}}{D_{0}} \frac{\partial^{2} w_{i}^{R}}{\partial t^{2}}\right) \\
& -y_{i}\left(\left(E_{b}-\frac{2 \tau_{0} \nu_{b}}{D_{0}}\right) \frac{\partial^{2} v_{i}^{R}}{\partial x_{i}^{2}}+\frac{2 \rho_{0} \nu_{b}}{D_{0}} \frac{\partial^{2} v_{i}^{R}}{\partial t^{2}}\right),
\end{aligned}
$$

where $D_{0}$ is the diameter of the CCNWs, $\nu_{b}$ and $E_{b}$ in order are the Poisson's ratio and the Young's modulus of the bulk, and $\rho_{0}$ is the mass per unit area of the surface layer.

\subsection{Equations of motion of magnetically affected DCCNWs using the RBT}

By employing the Newton's second law for both surface layer and bulk, the equations of motion in which describe transverse vibrations of the nanosystem in terms of the internal 
bending moments and the resultant shear forces are derived as follows:

$$
\begin{aligned}
& \frac{\partial M_{b_{z 1}}^{R}}{\partial x_{1}}+\int_{\mathcal{S}} y_{1} \frac{\partial \tau_{x x_{1}}^{R}}{\partial x_{1}} \mathrm{~d} \mathcal{S}-Q_{b_{y 1}}^{R}=\int_{A_{b}} \rho_{b} \frac{\partial^{2} u_{x_{1}}^{R}}{\partial t^{2}} y_{1} \mathrm{~d} A+\int_{\mathcal{S}} \rho_{0} \frac{\partial^{2} u_{x_{1}}^{R}}{\partial t^{2}} y_{1} \mathrm{~d} \mathcal{S}, \\
& -\frac{\partial Q_{b_{y 1}}^{R}}{\partial x}-\int_{\mathcal{S}} \frac{\partial \tau_{x y_{1}}^{R}}{\partial x} n_{y_{1}} \mathrm{~d} \mathcal{S}-T \frac{\partial^{2} v_{1}^{R}}{\partial x_{1}^{2}}=f_{m_{y 1}}^{R}-\int_{A_{b}} \rho_{b} \frac{\partial^{2} u_{y_{1}}^{R}}{\partial t^{2}} \mathrm{~d} A-\int_{\mathcal{S}} \rho_{0} \frac{\partial^{2} u_{y_{1}}^{R}}{\partial t^{2}} n_{y_{1}} \mathrm{~d} \mathcal{S} \\
& \frac{\partial M_{b_{y 1}}^{R}}{\partial x_{1}}+\int_{\mathcal{S}} z_{1} \frac{\partial \tau_{x x_{1}}^{R}}{\partial x_{1}} \mathrm{~d} \mathcal{S}-Q_{b_{z 1}}^{R}=\int_{A_{b}} \rho_{b} \frac{\partial^{2} u_{x_{1}}^{R}}{\partial t^{2}} z_{1} \mathrm{~d} A+\int_{\mathcal{S}} \rho_{0} \frac{\partial^{2} u_{x_{1}}^{R}}{\partial t^{2}} z_{1} \mathrm{~d} \mathcal{S}, \\
& -\frac{\partial Q_{b_{z 1}}^{R}}{\partial x}-\int_{\mathcal{S}} \frac{\partial \tau_{x z_{1}}^{R}}{\partial x} n_{z_{1}} \mathrm{~d} \mathcal{S}-T \frac{\partial^{2} w_{1}^{R}}{\partial x_{1}^{2}}=f_{m_{z 1}}^{R}-\int_{A_{b}} \rho_{b} \frac{\partial^{2} u_{z_{1}}^{R}}{\partial t^{2}} \mathrm{~d} A-\int_{\mathcal{S}} \rho_{0} \frac{\partial^{2} u_{z_{1}}^{R}}{\partial t^{2}} n_{z_{1}} \mathrm{~d} \mathcal{S} \\
& \frac{\partial M_{b_{z 2}}^{R}}{\partial x_{2}}+\int_{\mathcal{S}} y_{2} \frac{\partial \tau_{x x_{2}}^{R}}{\partial x_{2}} \mathrm{~d} \mathcal{S}-Q_{b_{y 2}}^{R}=\int_{A_{b}} \rho_{b} \frac{\partial^{2} u_{x_{2}}^{R}}{\partial t^{2}} y_{2} \mathrm{~d} A+\int_{\mathcal{S}} \rho_{0} \frac{\partial^{2} u_{x_{2}}^{R}}{\partial t^{2}} y_{2} \mathrm{~d} \mathcal{S}, \\
& -\frac{\partial Q_{b_{y 2}}^{R}}{\partial x}-\int_{\mathcal{S}} \frac{\partial \tau_{x y_{2}}^{R}}{\partial x} n_{y_{2}} \mathrm{~d} \mathcal{S}-T \frac{\partial^{2} v_{2}^{R}}{\partial x_{2}^{2}}=f_{m_{y^{2}}}^{R}-\int_{A_{b}} \rho_{b} \frac{\partial^{2} u_{y_{2}}^{R}}{\partial t^{2}} \mathrm{~d} A-\int_{\mathcal{S}} \rho_{0} \frac{\partial^{2} u_{y_{2}}^{R}}{\partial t^{2}} n_{y_{2}} \mathrm{~d} \mathcal{S} \\
& \frac{\partial M_{b_{y 2}}^{R}}{\partial x_{2}}+\int_{\mathcal{S}} z_{2} \frac{\partial \tau_{x x_{2}}^{R}}{\partial x_{2}} \mathrm{~d} \mathcal{S}-Q_{b_{z 2}}^{R}=\int_{A_{b}} \rho_{b} \frac{\partial^{2} u_{x_{2}}^{R}}{\partial t^{2}} z_{2} \mathrm{~d} A+\int_{\mathcal{S}} \rho_{0} \frac{\partial^{2} u_{x_{2}}^{R}}{\partial t^{2}} z_{2} \mathrm{~d} \mathcal{S}, \\
& -\frac{\partial Q_{b_{z 2}}^{R}}{\partial x}-\int_{\mathcal{S}} \frac{\partial \tau_{x z_{2}}^{R}}{\partial x} n_{z_{2}} \mathrm{~d} \mathcal{S}-T \frac{\partial^{2} w_{2}^{R}}{\partial x_{2}^{2}}=f_{m_{z 2}}^{R}-\int_{A_{b}} \rho_{b} \frac{\partial^{2} u_{z_{2}}^{R}}{\partial t^{2}} \mathrm{~d} A-\int_{\mathcal{S}} \rho_{0} \frac{\partial^{2} u_{z_{2}}^{R}}{\partial t^{2}} n_{z_{2}} \mathrm{~d} \mathcal{S}
\end{aligned}
$$

where $M_{b_{y_{i}}}^{R} / M_{b_{z_{i}}}^{R}$ and $Q_{b_{y_{i}}}^{R} / Q_{b_{z_{i}}}^{R}$ are the bending moments and the shear forces of the bulk of $i$ th CCNW associated with the $y_{i}$ and $z_{i}$ axes, respectively, and $\mathrm{d} \mathcal{S}$ is the length of an infinitesimal segment of the perimeter of the cross-section of CCNWs. By mixing Eqs. (20a), (20c), (20e), and (20g) with Eqs. (20b), (20d), (20f), and (20h), respectively, and defining the bending moments of the CCNWs on the basis of the RBT as follows:

$$
M_{b_{y i}}^{R}=\int_{A} z_{i} \sigma_{x x_{i}}^{R} \mathrm{~d} A=-\left(E_{b} I_{b}-\frac{2 \tau_{0} \nu_{b} I_{b}}{D_{0}}\right) \frac{\partial^{2} w_{i}^{R}}{\partial x_{i}^{2}}-\frac{2 \nu_{b} I_{b} \tau_{0}}{D_{0}} \frac{\partial^{2} w_{i}^{R}}{\partial t^{2}},
$$




$$
M_{b_{z i}}^{R}=\int_{A} y_{i} \sigma_{x x_{i}}^{R} \mathrm{~d} A=-\left(E_{b} I_{b}-\frac{2 \tau_{0} \nu_{b} I_{b}}{D_{0}}\right) \frac{\partial^{2} v_{i}^{R}}{\partial x_{i}^{2}}-\frac{2 \nu_{b} I_{b} \tau_{0}}{D_{0}} \frac{\partial^{2} v_{i}^{R}}{\partial t^{2}}
$$

the equations of motion of the current-carrying nanosystem subjected to a longitudinal magnetic field in terms of the transverse displacements and the dynamic Lorentz's magnetic forces are obtained as:

$$
\begin{aligned}
& \left(\rho_{b} A_{b}+\rho_{0} S_{0}^{*}\right) \frac{\partial^{2} v_{1}^{R}}{\partial t^{2}}-\left(\rho_{b} I_{b}+\rho_{0} I_{0}^{*}-\frac{2 \nu_{b} I_{b} \rho_{0}}{D_{0}}\right) \frac{\partial^{4} v_{1}^{R}}{\partial t^{2} \partial x_{1}^{2}}+ \\
& {\left[E_{b} I_{b}+\left(\lambda_{0}+2 \mu_{0}\right) I_{0}^{*}-\frac{2 \nu_{b} I_{b} \tau_{0}}{D_{0}}\right] \frac{\partial^{4} v_{1}^{R}}{\partial x_{1}^{4}}-\left(T+\tau_{0} S_{0}^{*}\right) \frac{\partial^{2} v_{1}^{R}}{\partial x_{1}^{2}}=f_{m_{y 1}}^{R},} \\
& \left(\rho_{b} A_{b}+\rho_{0} S_{0}^{*}\right) \frac{\partial^{2} w_{1}^{R}}{\partial t^{2}}-\left(\rho_{b} I_{b}+\rho_{0} I_{0}^{*}-\frac{2 \nu_{b} I_{b} \rho_{0}}{D_{0}}\right) \frac{\partial^{4} w_{1}^{R}}{\partial t^{2} \partial x_{1}^{2}}+ \\
& {\left[E_{b} I_{b}+\left(\lambda_{0}+2 \mu_{0}\right) I_{0}^{*}-\frac{2 \nu_{b} I_{b} \tau_{0}}{D_{0}}\right] \frac{\partial^{4} w_{1}^{R}}{\partial x_{1}^{4}}-\left(T+\tau_{0} S_{0}^{*}\right) \frac{\partial^{2} w_{1}^{R}}{\partial x_{2}^{2}}=f_{m_{z 1} 1}^{R},} \\
& \left(\rho_{b} A_{b}+\rho_{0} S_{0}^{*}\right) \frac{\partial^{2} v_{2}^{R}}{\partial t^{2}}-\left(\rho_{b} I_{b}+\rho_{0} I_{0}^{*}-\frac{2 \nu_{b} I_{b} \rho_{0}}{D_{0}}\right) \frac{\partial^{4} v_{2}^{R}}{\partial t^{2} \partial x_{2}^{2}+} \\
& {\left[E_{b} I_{b}+\left(\lambda_{0}+2 \mu_{0}\right) I_{0}^{*}-\frac{2 \nu_{b} I_{b} \tau_{0}}{D_{0}}\right] \frac{\partial^{4} v_{2}^{R}}{\partial x_{2}^{4}}-\left(T+\tau_{0} S_{0}^{*}\right) \frac{\partial^{2} v_{2}^{R}}{\partial x_{2}^{2}}=f_{m_{y 2}}^{R},} \\
& \left(\rho_{b} A_{b}+\rho_{0} S_{0}^{*}\right) \frac{\partial^{2} w_{2}^{R}}{\partial t^{2}}-\left(\rho_{b} I_{b}+\rho_{0} I_{0}^{*}-\frac{2 \nu_{b} I_{b} \rho_{0}}{D_{0}}\right) \frac{\partial^{4} w_{2}^{R}}{\partial t^{2} \partial x_{2}^{2}}+ \\
& {\left[E_{b} I_{b}+\left(\lambda_{0}+2 \mu_{0}\right) I_{0}^{*}-\frac{2 \nu_{b} I_{b} \tau_{0}}{D_{0}}\right] \frac{\partial^{4} w_{2}^{R}}{\partial x_{2}^{4}}-\left(T+\tau_{0} S_{0}^{*}\right) \frac{\partial^{2} w_{2}^{R}}{\partial x_{2}^{2}}=f_{m_{z 2}}^{R},} \\
& {[}
\end{aligned}
$$

where $I_{0}^{*}=\int_{\mathcal{S}} z^{2} \mathrm{~d} \mathcal{S}$ and $S_{0}^{*}=\int_{\mathcal{S}} n_{z}^{2} \mathrm{~d} \mathcal{S}$.

\subsubsection{An approximate version of the governing equations based on the RBT}

To construct the approximate equations of motion, the approximate components of the magnetic forces are introduced to Eqs. (22a)-(22d). By substituting Eqs. (10a) and (10b) into these equations, the approximate version of the governing equations in terms of the 
transverse displacement fields of the CCNWs and their derivatives is readily obtainable as:

$$
\begin{aligned}
& \left(\rho_{b} A_{b}+\rho_{0} S_{0}^{*}\right) \frac{\partial^{2} v_{1}^{R}}{\partial t^{2}}-\left(\rho_{b} I_{b}+\rho_{0} I_{0}^{*}-\frac{2 \nu_{b} I_{b} \rho_{0}}{D_{0}}\right) \frac{\partial^{4} v_{1}^{R}}{\partial t^{2} \partial x_{1}^{2}}+ \\
& {\left[E_{b} I_{b}+\left(\lambda_{0}+2 \mu_{0}\right) I_{0}^{*}-\frac{2 \nu_{b} I_{b} \tau_{0}}{D_{0}}\right] \frac{\partial^{4} v_{1}^{R}}{\partial x_{1}^{4}}-\left(T+\tau_{0} S_{0}^{*}\right) \frac{\partial^{2} v_{1}^{R}}{\partial x_{1}^{2}}} \\
& -B_{0} I_{0} \frac{\partial w_{1}^{R}}{\partial x_{1}}+\frac{\mu_{0} I_{0}^{2}}{2 \pi d^{2}}\left(v_{2}^{R}-v_{1}^{R}\right)=0, \\
& \left(\rho_{b} A_{b}+\rho_{0} S_{0}^{*}\right) \frac{\partial^{2} w_{1}^{R}}{\partial t^{2}}-\left(\rho_{b} I_{b}+\rho_{0} I_{0}^{*}-\frac{2 \nu_{b} I_{b} \rho_{0}}{D_{0}}\right) \frac{\partial^{4} w_{1}^{R}}{\partial t^{2} \partial x_{1}^{2}}+ \\
& {\left[E_{b} I_{b}+\left(\lambda_{0}+2 \mu_{0}\right) I_{0}^{*}-\frac{2 \nu_{b} I_{b} \tau_{0}}{D_{0}}\right] \frac{\partial^{4} w_{1}^{R}}{\partial x_{1}^{4}}-\left(T+\tau_{0} S_{0}^{*}\right) \frac{\partial^{2} w_{1}^{R}}{\partial x_{1}^{2}}+} \\
& B_{0} I_{0} \frac{\partial v_{1}^{R}}{\partial x_{1}}-\frac{\mu_{0} I_{0}^{2}}{2 \pi d^{2}}\left(w_{2}^{R}-w_{1}^{R}\right)=0, \\
& \left(\rho_{b} A_{b}+\rho_{0} S_{0}^{*}\right) \frac{\partial^{2} v_{2}^{R}}{\partial t^{2}}-\left(\rho_{b} I_{b}+\rho_{0} I_{0}^{*}-\frac{2 \nu_{b} I_{b} \rho_{0}}{D_{0}}\right) \frac{\partial^{4} v_{2}^{R}}{\partial t^{2} \partial x_{2}^{2}}+ \\
& {\left[E_{b} I_{b}+\left(\lambda_{0}+2 \mu_{0}\right) I_{0}^{*}-\frac{2 \nu_{b} I_{b} \tau_{0}}{D_{0}}\right] \frac{\partial^{4} v_{2}^{R}}{\partial x_{2}^{4}}-\left(T+\tau_{0} S_{0}^{*}\right) \frac{\partial^{2} v_{2}^{R}}{\partial x_{2}^{2}}+} \\
& B_{0} I_{0} \frac{\partial w_{2}^{R}}{\partial x_{2}}-\frac{\mu_{0} I_{0}^{2}}{2 \pi d^{2}}\left(v_{2}^{R}-v_{1}^{R}\right)=0 \\
& \left(\rho_{b} A_{b}+\rho_{0} S_{0}^{*}\right) \frac{\partial^{2} w_{2}^{R}}{\partial t^{2}}-\left(\rho_{b} I_{b}+\rho_{0} I_{0}^{*}-\frac{2 \nu_{b} I_{b} \rho_{0}}{D_{0}}\right) \frac{\partial^{4} w_{2}^{R}}{\partial t^{2} \partial x_{2}^{2}}+ \\
& {\left[E_{b} I_{b}+\left(\lambda_{0}+2 \mu_{0}\right) I_{0}^{*}-\frac{2 \nu_{b} I_{b} \tau_{0}}{D_{0}}\right] \frac{\partial^{4} w_{2}^{R}}{\partial x_{2}^{4}}-\left(T+\tau_{0} S_{0}^{*}\right) \frac{\partial^{2} w_{2}^{R}}{\partial x_{2}^{2}}-} \\
& B_{0} I_{0} \frac{\partial v_{2}^{R}}{\partial x_{2}}+\frac{\mu_{0} I_{0}^{2}}{2 \pi d^{2}}\left(w_{2}^{R}-w_{1}^{R}\right)=0 .
\end{aligned}
$$

In order to examine vibration of the nanosystem more conveniently, the following dimensionless quantities are considered:

$$
\begin{aligned}
& \bar{v}_{i}^{R}=\frac{v_{i}^{R}}{l_{b}}, \bar{w}_{i}^{R}=\frac{w_{i}^{R}}{l_{b}}, \xi_{i}=\frac{x_{i}}{l_{b}}, \tau=\frac{t}{l_{b}^{2}} \sqrt{\frac{E_{b} I_{b}}{\rho_{b} A_{b}}}, \lambda=\frac{l_{b}}{r_{b}}, \\
& \bar{T}^{R}=\frac{T l_{b}^{2}}{E_{b} I_{b}}, \bar{f}_{0}^{R}=\frac{B_{0} I_{0} l_{b}^{3}}{E_{b} I_{b}}, \bar{f}_{1}^{R}=\frac{\mu_{0} I_{0}^{2} l_{b}^{4}}{2 \pi d^{2} E_{b} I_{b}}, \chi_{1}^{R}=\frac{\rho_{0} S_{0}^{*}}{\rho_{b} A_{b}}, \\
& \chi_{2}^{R}=\frac{\rho_{0} I_{0}^{*}-\frac{2 \nu_{b} I_{b} \rho_{0}}{D_{0}}}{\rho_{b} I_{b}}, \chi_{3}^{R}=\frac{\left(\lambda_{0}+2 \mu_{0}\right) I_{0}^{*}-\frac{2 \nu_{b} I_{b} \tau_{0}}{D_{0}}}{E_{b} I_{b}}, \chi_{4}^{R}=\frac{\tau_{0} S_{0}^{*} l_{b}^{2}}{E_{b} I_{b}},
\end{aligned}
$$


by introducing Eq. (24) to Eqs. (23a)-(23d), one can arrive at the approximate version of the dimensionless equations of motion of magnetically affected DCCNWs on the basis of the RBT:

$$
\begin{aligned}
& \left(1+\chi_{1}^{R}\right) \frac{\partial^{2} \bar{v}_{1}^{R}}{\partial \tau^{2}}-\lambda^{-2}\left(1+\chi_{2}^{R}\right) \frac{\partial^{4} \bar{v}_{1}^{R}}{\partial \tau^{2} \partial \xi_{1}^{2}}+ \\
& \left(1+\chi_{3}^{R}\right) \frac{\partial^{4} \bar{v}_{1}^{R}}{\partial \xi_{1}^{4}}-\left(\bar{T}^{R}+\chi_{4}^{R}\right) \frac{\partial^{2} \bar{v}_{1}^{R}}{\partial \xi_{1}^{2}}-\bar{f}_{0}^{R} \frac{\partial \bar{w}_{1}^{R}}{\partial \xi_{1}}+\bar{f}_{1}^{R}\left(\bar{v}_{2}^{R}-\bar{v}_{1}^{R}\right)=0 \\
& \left(1+\chi_{1}^{R}\right) \frac{\partial^{2} \bar{w}_{1}^{R}}{\partial \tau^{2}}-\lambda^{-2}\left(1+\chi_{2}^{R}\right) \frac{\partial^{4} \bar{w}_{1}^{R}}{\partial \tau^{2} \partial \xi_{1}^{2}}+ \\
& \left(1+\chi_{3}^{R}\right) \frac{\partial^{4} \bar{w}_{1}^{R}}{\partial \xi_{1}^{4}}-\left(\bar{T}^{R}+\chi_{4}^{R}\right) \frac{\partial^{2} \bar{w}_{1}^{R}}{\partial \xi_{1}^{2}}+\bar{f}_{0}^{R} \frac{\partial \bar{v}_{1}^{R}}{\partial \xi_{1}}-\bar{f}_{1}^{R}\left(\bar{w}_{2}^{R}-\bar{w}_{1}^{R}\right)=0 \\
& \left(1+\chi_{1}^{R}\right) \frac{\partial^{2} \bar{v}_{2}^{R}}{\partial \tau^{2}}-\lambda^{-2}\left(1+\chi_{2}^{R}\right) \frac{\partial^{4} \bar{v}_{2}^{R}}{\partial \tau^{2} \partial \xi_{2}^{2}}+ \\
& \left(1+\chi_{3}^{R}\right) \frac{\partial^{4} \bar{v}_{2}^{R}}{\partial \xi_{2}^{4}}-\left(\bar{T}^{R}+\chi_{4}^{R}\right) \frac{\partial^{2} \bar{v}_{2}^{R}}{\partial \xi_{2}^{2}}-\bar{f}_{0}^{R} \frac{\partial \bar{w}_{2}^{R}}{\partial \xi_{2}}-\bar{f}_{1}^{R}\left(\bar{v}_{2}^{R}-\bar{v}_{1}^{R}\right)=0 \\
& \left(1+\chi_{1}^{R}\right) \frac{\partial^{2} \bar{w}_{2}^{R}}{\partial \tau^{2}}-\lambda^{-2}\left(1+\chi_{2}^{R}\right) \frac{\partial^{4} \bar{w}_{2}^{R}}{\partial \tau^{2} \partial \xi_{2}^{2}}+ \\
& \left(1+\chi_{3}^{R}\right) \frac{\partial^{4} \bar{w}_{2}^{R}}{\partial \xi_{2}^{4}}-\left(\bar{T}^{R}+\chi_{4}^{R}\right) \frac{\partial^{2} \bar{w}_{2}^{R}}{\partial \xi_{2}^{2}}+\bar{f}_{0}^{R} \frac{\partial \bar{v}_{2}^{R}}{\partial \xi_{2}}+\bar{f}_{1}^{R}\left(\bar{w}_{2}^{R}-\bar{w}_{1}^{R}\right)=0
\end{aligned}
$$

By setting $\chi_{i}^{R}=0 ; i=1,2,3,4$, Eqs. (25a)-(25d) reduce to the governing equations of doubly parallel current-carrying macrowires in the presence of longitudinal magnetic field. For very lengthy nanowires (i.e., in the absence of bending rigidity and rotational inertia), the dynamic response of the nanosystem under arbitrary initial conditions was explored analytically by Kiani $(2015 c, d)$. The explicit expressions of the frequencies and their corresponding vibration modes were obtained. Furthermore, the conditions which lead to the dynamic instability of the nanosystem were explained and discussed.

\subsubsection{An exact version of the governing equations based on the RBT}

To obtain the exact equations of motion, a more refined version of the magnetic forces obtained in the previous part is exploited. By substituting Eqs. (14a) and (14b) into Eqs. (22a)(22d), the exact governing equations of the DCCNWs immersed in a longitudinal magnetic 
field according to the RBT are derived as follows:

$$
\begin{aligned}
& \left(\rho_{b} A_{b}+\rho_{0} S_{0}^{*}\right) \frac{\partial^{2} v_{1}^{R}}{\partial t^{2}}-\left(\rho_{b} I_{b}+\rho_{0} I_{0}^{*}-\frac{2 \nu_{b} I_{b} \rho_{0}}{D_{0}}\right) \frac{\partial^{4} v_{1}^{R}}{\partial t^{2} \partial x_{1}^{2}}+ \\
& {\left[E_{b} I_{b}+\left(\lambda_{0}+2 \mu_{0}\right) I_{0}^{*}-\frac{2 \nu_{b} I_{b} \tau_{0}}{D_{0}}\right] \frac{\partial^{4} v_{1}^{R}}{\partial x_{1}^{4}}-\left(T+\tau_{0} S_{0}^{*}\right) \frac{\partial^{2} v_{1}^{R}}{\partial x_{1}^{2}}-} \\
& B_{0} I_{0} \frac{\partial w_{1}^{R}}{\partial x_{1}}+\frac{\mu_{0} I_{0}^{2}}{4 \pi} \int_{0}^{l_{b}} \frac{\left(x_{2}-x_{1}\right) \frac{\partial v_{2}^{R}}{\partial x_{2}}-\left(v_{2}^{R}-v_{1}^{R}\right)}{\left(\left(x_{2}-x_{1}\right)^{2}+d^{2}\right)^{\frac{3}{2}}} \mathrm{~d} x_{2}=0, \\
& \left(\rho_{b} A_{b}+\rho_{0} S_{0}^{*}\right) \frac{\partial^{2} w_{1}^{R}}{\partial t^{2}}-\left(\rho_{b} I_{b}+\rho_{0} I_{0}^{*}-\frac{2 \nu_{b} I_{b} \rho_{0}}{D_{0}}\right) \frac{\partial^{4} w_{1}^{R}}{\partial t^{2} \partial x_{1}^{2}}+ \\
& {\left[E_{b} I_{b}+\left(\lambda_{0}+2 \mu_{0}\right) I_{0}^{*}-\frac{2 \nu_{b} I_{b} \tau_{0}}{D_{0}}\right] \frac{\partial^{4} w_{1}^{R}}{\partial x_{1}^{4}}-\left(T+\tau_{0} S_{0}^{*}\right) \frac{\partial^{2} w_{1}^{R}}{\partial x_{1}^{2}}+} \\
& B_{0} I_{0} \frac{\partial v_{1}^{R}}{\partial x_{1}}+\frac{\mu_{0} I_{0}^{2}}{4 \pi} \int_{0}^{l_{b}} \frac{\left(x_{2}-x_{1}\right) \frac{\partial w_{2}^{R}}{\partial x_{2}}-\left(w_{2}^{R}-w_{1}^{R}\right)}{\left(\left(x_{2}-x_{1}\right)^{2}+d^{2}\right)^{\frac{3}{2}}} \mathrm{~d} x_{2}=0, \\
& \left(\rho_{b} A_{b}+\rho_{0} S_{0}^{*}\right) \frac{\partial^{2} v_{2}^{R}}{\partial t^{2}}-\left(\rho_{b} I_{b}+\rho_{0} I_{0}^{*}-\frac{2 \nu_{b} I_{b} \rho_{0}}{D_{0}}\right) \frac{\partial^{4} v_{2}^{R}}{\partial t^{2} \partial x_{2}^{2}}+ \\
& {\left[E_{b} I_{b}+\left(\lambda_{0}+2 \mu_{0}\right) I_{0}^{*}-\frac{2 \nu_{b} I_{b} \tau_{0}}{D_{0}}\right] \frac{\partial^{4} v_{2}^{R}}{\partial x_{2}^{4}}-\left(T+\tau_{0} S_{0}^{*}\right) \frac{\partial^{2} v_{2}^{R}}{\partial x_{2}^{2}}-} \\
& B_{0} I_{0} \frac{\partial w_{2}^{R}}{\partial x_{2}}-\frac{\mu_{0} I_{0}^{2}}{4 \pi} \int_{0}^{l_{b}} \frac{\left(x_{2}-x_{1}\right) \frac{\partial v_{1}^{R}}{\partial x_{1}}-\left(v_{2}^{R}-v_{1}^{R}\right)}{\left(\left(x_{2}-x_{1}\right)^{2}+d^{2}\right)^{\frac{3}{2}}} \mathrm{~d} x_{1}=0, \\
& \left(\rho_{b} A_{b}+\rho_{0} S_{0}^{*}\right) \frac{\partial^{2} w_{2}^{R}}{\partial t^{2}}-\left(\rho_{b} I_{b}+\rho_{0} I_{0}^{*}-\frac{2 \nu_{b} I_{b} \rho_{0}}{D_{0}}\right) \frac{\partial^{4} w_{2}^{R}}{\partial t^{2} \partial x_{2}^{2}}+ \\
& {\left[E_{b} I_{b}+\left(\lambda_{0}+2 \mu_{0}\right) I_{0}^{*}-\frac{2 \nu_{b} I_{b} \tau_{0}}{D_{0}}\right] \frac{\partial^{4} w_{2}^{R}}{\partial x_{2}^{4}}-\left(T+\tau_{0} S_{0}^{*}\right) \frac{\partial^{2} w_{2}^{R}}{\partial x_{2}^{2}}+} \\
& B_{0} I_{0} \frac{\partial v_{2}^{R}}{\partial x_{2}}-\frac{\mu_{0} I_{0}^{2}}{4 \pi} \int_{0}^{l_{b}} \frac{\left(x_{2}-x_{1}\right) \frac{\partial w_{1}^{R}}{\partial x_{1}}-\left(w_{2}^{R}-w_{1}^{R}\right)}{\left(\left(x_{2}-x_{1}\right)^{2}+d^{2}\right)^{\frac{3}{2}}} \mathrm{~d} x_{1}=0 .
\end{aligned}
$$

By introducing the dimensionless quantities in Eq. (24) to Eqs. (26a)-(26d), the dimensionless equations of motion of magnetically affected DCCNWs based on the RBT and a more 
refined model of the dynamical magnetic force are obtained as:

$$
\begin{aligned}
& \left(1+\chi_{1}^{R}\right) \frac{\partial^{2} \bar{v}_{1}^{R}}{\partial \tau^{2}}-\lambda^{-2}\left(1+\chi_{2}^{R}\right) \frac{\partial^{4} \bar{v}_{1}^{R}}{\partial \tau^{2} \partial \xi_{1}^{2}}+\left(1+\chi_{3}^{R}\right) \frac{\partial^{4} \bar{v}_{1}^{R}}{\partial \xi_{1}^{4}}- \\
& \left(\bar{T}^{R}+\chi_{4}^{R}\right) \frac{\partial^{2} \bar{v}_{1}^{R}}{\partial \xi_{1}^{2}}-\bar{f}_{0}^{R} \frac{\partial \bar{w}_{1}^{R}}{\partial \xi_{1}}+\frac{\bar{f}_{1}^{R}}{2 \bar{d}} \int_{0}^{1} \frac{\left(\xi_{2}-\xi_{1}\right) \frac{\partial \bar{v}_{2}^{R}}{\partial \xi_{2}}-\left(\bar{v}_{2}^{R}-\bar{v}_{1}^{R}\right)}{\left(\left(\frac{\xi_{2}-\xi_{1}}{\bar{d}}\right)^{2}+1\right)^{\frac{3}{2}}} \mathrm{~d} \xi_{2}=0 \\
& \left(1+\chi_{1}^{R}\right) \frac{\partial^{2} \bar{w}_{1}^{R}}{\partial \tau^{2}}-\lambda^{-2}\left(1+\chi_{2}^{R}\right) \frac{\partial^{4} \bar{w}_{1}^{R}}{\partial \tau^{2} \partial \xi_{1}^{2}}+\left(1+\chi_{3}^{R}\right) \frac{\partial^{4} \bar{w}_{1}^{R}}{\partial \xi_{1}^{4}}- \\
& \left(\bar{T}^{R}+\chi_{4}^{R}\right) \frac{\partial^{2} \bar{w}_{1}^{R}}{\partial \xi_{1}^{2}}+\bar{f}_{0}^{R} \frac{\partial \bar{v}_{1}^{R}}{\partial \xi_{1}}+\frac{\bar{f}_{1}^{R}}{2 \bar{d}} \int_{0}^{1} \frac{\left(\xi_{2}-\xi_{1}\right) \frac{\partial \bar{w}_{2}^{R}}{\partial \xi_{2}}-\left(\bar{w}_{2}^{R}-\bar{w}_{1}^{R}\right)}{\left(\left(\frac{\xi_{2}-\xi_{1}}{\bar{d}}\right)^{2}+1\right)^{\frac{3}{2}}} \mathrm{~d} \xi_{2}=0, \\
& \left(1+\chi_{1}^{R}\right) \frac{\partial^{2} \bar{v}_{2}^{R}}{\partial \tau^{2}}-\lambda^{-2}\left(1+\chi_{2}^{R}\right) \frac{\partial^{4} \bar{v}_{2}^{R}}{\partial \tau^{2} \partial \xi_{2}^{2}}+\left(1+\chi_{3}^{R}\right) \frac{\partial^{4} \bar{v}_{2}^{R}}{\partial \xi_{2}^{4}}- \\
& \left(\bar{T}^{R}+\chi_{4}^{R}\right) \frac{\partial^{2} \bar{v}_{2}^{R}}{\partial \xi_{2}^{2}}-\bar{f}_{0}^{R} \frac{\partial \bar{w}_{2}^{R}}{\partial \xi_{2}}-\frac{\bar{f}_{1}^{R}}{2 \bar{d}} \int_{0}^{1} \frac{\left(\xi_{2}-\xi_{1}\right) \frac{\partial \bar{v}_{1}^{R}}{\partial \xi_{1}}-\left(\bar{v}_{2}^{R}-\bar{v}_{1}^{R}\right)}{\left(\left(\frac{\xi_{2}-\xi_{1}}{\bar{d}}\right)^{2}+1\right)^{\frac{3}{2}}} \mathrm{~d} \xi_{1}=0 \\
& \left(1+\chi_{1}^{R}\right) \frac{\partial^{2} \bar{w}_{2}^{R}}{\partial \tau^{2}}-\lambda^{-2}\left(1+\chi_{2}^{R}\right) \frac{\partial^{4} \bar{w}_{2}^{R}}{\partial \tau^{2} \partial \xi_{2}^{2}}+\left(1+\chi_{3}^{R}\right) \frac{\partial^{4} \bar{w}_{2}^{R}}{\partial \xi_{2}^{4}}- \\
& \left(\bar{T}^{R}+\chi_{4}^{R}\right) \frac{\partial^{2} \bar{w}_{2}^{R}}{\partial \xi_{2}^{2}}+\bar{f}_{0}^{R} \frac{\partial \bar{v}_{2}^{R}}{\partial \xi_{2}}-\frac{\bar{f}_{1}^{R}}{2 \bar{d}} \int_{0}^{1} \frac{\left(\xi_{2}-\xi_{1}\right) \frac{\partial \bar{w}_{1}^{R}}{\partial \xi_{1}}-\left(\bar{w}_{2}^{R}-\bar{w}_{1}^{R}\right)}{\left(\left(\frac{\xi_{2}-\xi_{1}}{\bar{d}}\right)^{2}+1\right)^{\frac{3}{2}}} \mathrm{~d} \xi_{1}=0 .
\end{aligned}
$$

Eqs. (27a)-(27d) represent four coupled integro-partial differential equations (IPDE) that should be appropriately solved to study free vibration of the nanosystem based on the RBT.

\subsection{Vibration of the magnetically affected nanosystem based on the RBT via RKPM}

\subsubsection{Free transverse vibration on the basis of the proposed approximate model}

To study free dynamic response of magnetically affected DCCNWs at hand, the dimensionless transverse displacements of the NWs based on the RBT are discretized as follows:

$$
\bar{v}_{i}^{R}\left(\xi_{i}, \tau\right)=\sum_{I=1}^{N P_{i}} \phi_{I}^{v_{i}}\left(\xi_{i}\right) \bar{v}_{i_{I}}^{R}(\tau), \bar{w}^{R}\left(\xi_{i}, \tau\right)=\sum_{I=1}^{N P_{i}} \phi_{I}^{w_{i}}\left(\xi_{i}\right) \bar{w}_{i_{I}}^{R}(\tau) ; i=1,2,
$$


where $N P_{i}$ is the number of RKPM's particles associated with the $i$ th $\mathrm{CCNW}, \phi_{I}^{v_{i}}(\xi)$ and $\phi_{I}^{w_{i}}(\xi)$ in order are the RKPM's shape functions of the $I$ th particle pertinent to the dimensionless fields $\bar{v}_{i}^{R}$ and $\bar{w}_{i}^{R}, \bar{v}_{i_{I}}^{R}(\tau)$ and $\bar{w}_{i_{I}}^{R}(\tau)$ are their dynamic nodal parameter values. To discretize the dimensionless equations of motion in Eqs. (25a)-(25d), these equations are premultiplied by $\delta \bar{v}_{1}^{R}, \delta \bar{w}_{1}^{R}, \delta \bar{v}_{2}^{R}$, and $\delta \bar{w}_{2}^{R}$, respectively, where $\delta$ denotes the variational sign. After taking the integral from the resulting expressions in the dimensionless spatial domain of the CCNWs (i.e., $[0,1]$ ), and taking the required integration by parts, the following set of equations is readily extractable:

$$
\begin{gathered}
{\left[\begin{array}{llll}
{\left[\overline{\mathbf{M}}_{b}^{R}\right]^{v_{1} v_{1}}} & {\left[\overline{\mathbf{M}}_{b}^{R}\right]^{v_{1} w_{1}}} & {\left[\overline{\mathbf{M}}_{b}^{R}\right]^{v_{1} v_{2}}} & {\left[\overline{\mathbf{M}}_{b}^{R}\right]^{v_{1} w_{2}}} \\
{\left[\overline{\mathbf{M}}_{b}^{R}\right]^{w_{1} v_{1}}} & {\left[\overline{\mathbf{M}}_{b}^{R}\right]^{w_{1} w_{1}}} & {\left[\overline{\mathbf{M}}_{b}^{R}\right]^{w_{1} v_{2}}} & {\left[\overline{\mathbf{M}}_{b}^{R}\right]^{w_{1} w_{2}}} \\
{\left[\overline{\mathbf{M}}_{b}^{R}\right]^{v_{2} v_{1}}} & {\left[\overline{\mathbf{M}}_{b}^{R}\right]^{v_{2} w_{1}}} & {\left[\overline{\mathbf{M}}_{b}^{R}\right]^{v_{2} v_{2}}} & {\left[\overline{\mathbf{M}}_{b}^{R}\right]^{v_{2} w_{2}}} \\
{\left[\overline{\mathbf{M}}_{b}^{R}\right]^{w_{2} v_{1}}} & {\left[\overline{\mathbf{M}}_{b}^{R}\right]^{w_{2} w_{1}}} & {\left[\overline{\mathbf{M}}_{b}^{R}\right]^{w_{2} v_{2}}} & {\left[\overline{\mathbf{M}}_{b}^{R}\right]^{w_{2} w_{2}}}
\end{array}\right]\left\{\begin{array}{c}
\frac{\mathrm{d}^{2} \overline{\mathbf{v}}_{1}^{R}}{\mathrm{~d} \tau^{2}} \\
\frac{\mathrm{d}^{2} \overline{\mathbf{w}}_{1}^{R}}{\mathrm{~d} \tau^{2}} \\
\frac{\mathrm{d}^{2} \overline{\mathbf{v}}_{2}^{R}}{\mathrm{~d} \tau^{2}} \\
\frac{\mathrm{d}^{2} \overline{\mathbf{w}}_{2}^{R}}{\mathrm{~d} \tau^{2}}
\end{array}\right\}+} \\
{\left[\begin{array}{llll}
{\left[\overline{\mathbf{K}}_{b}^{R}\right]^{v_{1} v_{1}}} & {\left[\overline{\mathbf{K}}_{b}^{R}\right]^{v_{1} w_{1}}} & {\left[\overline{\mathbf{K}}_{b}^{R}\right]^{v_{1} v_{2}}} & {\left[\overline{\mathbf{K}}_{b}^{R}\right]^{v_{1} w_{2}}} \\
{\left[\overline{\mathbf{K}}_{b}^{R}\right]^{w_{1} v_{1}}} & {\left[\overline{\mathbf{K}}_{b}^{R}\right]^{w_{1} w_{1}}} & {\left[\overline{\mathbf{K}}_{b}^{R}\right]^{w_{1} v_{2}}} & {\left[\overline{\mathbf{K}}_{b}^{R}\right]^{w_{1} w_{2}}} \\
{\left[\overline{\mathbf{K}}_{b}^{R}\right]^{v_{2} v_{1}}} & {\left[\overline{\mathbf{K}}_{b}^{R}\right]^{v_{2} w_{1}}} & {\left[\overline{\mathbf{K}}_{b}^{R}\right]^{v_{2} v_{2}}} & {\left[\overline{\mathbf{K}}_{b}^{R}\right]^{v_{2} w_{2}}} \\
{\left[\overline{\mathbf{K}}_{b}^{R}\right]^{w_{2} v_{1}}} & {\left[\overline{\mathbf{K}}_{b}^{R}\right]^{w_{2} w_{1}}} & {\left[\overline{\mathbf{K}}_{b}^{R}\right]^{w_{2} v_{2}}} & {\left[\overline{\mathbf{K}}_{b}^{R}\right]^{w_{2} w_{2}}}
\end{array}\right]\left\{\begin{array}{c}
\overline{\mathbf{v}}_{1}^{R} \\
\overline{\mathbf{w}}_{1}^{R} \\
\overline{\mathbf{v}}_{2}^{R} \\
\overline{\mathbf{w}}_{2}^{R}
\end{array}\right\}=\left\{\begin{array}{c}
\mathbf{0} \\
\mathbf{0} \\
\mathbf{0} \\
\mathbf{0}
\end{array}\right\},}
\end{gathered}
$$

where the nonzero dimensionless mass and stiffness submatrices as well as the dimensionless nodal parameter vectors are given by:

$$
\begin{gathered}
{\left[\overline{\mathbf{M}}_{b}^{R}\right]_{I J}^{v_{i} v_{i}}=\int_{0}^{1}\left(\left(1+\chi_{1}^{R}\right) \phi_{I}^{v_{i}} \phi_{J}^{v_{i}}+\lambda^{-2}\left(1+\chi_{2}^{R}\right) \frac{\mathrm{d} \phi_{I}^{v_{i}}}{\mathrm{~d} \xi_{i}} \frac{\mathrm{d} \phi_{J}^{v_{i}}}{\mathrm{~d} \xi_{i}}\right) \mathrm{d} \xi_{i}} \\
{\left[\overline{\mathbf{M}}_{b}^{R}\right]_{I J}^{w_{i} w_{i}}=\int_{0}^{1}\left(\left(1+\chi_{1}^{R}\right) \phi_{I}^{w_{i}} \phi_{J}^{w_{i}}+\lambda^{-2}\left(1+\chi_{2}^{R}\right) \frac{\mathrm{d} \phi_{I}^{w_{i}}}{\mathrm{~d} \xi_{i}} \frac{\mathrm{d} \phi_{J}^{w_{i}}}{\mathrm{~d} \xi_{i}}\right) \mathrm{d} \xi_{i}} \\
{\left[\overline{\mathbf{K}}_{b}^{R}\right]_{I J}^{v_{i} v_{i}}=\int_{0}^{1}\left(\left(1+\chi_{3}^{R}\right) \frac{\mathrm{d}^{2} \phi_{I}^{v_{i}}}{\mathrm{~d} \xi_{i}^{2}} \frac{\mathrm{d}^{2} \phi_{J}^{v_{i}}}{\mathrm{~d} \xi_{i}^{2}}+\left(\bar{T}^{R}+\chi_{4}^{R}\right) \frac{\mathrm{d} \phi_{I}^{v_{i}}}{\mathrm{~d} \xi_{i}} \frac{\mathrm{d} \phi_{J}^{v_{i}}}{\mathrm{~d} \xi_{i}}-\bar{f}_{1}^{R} \phi_{I}^{v_{i}} \phi_{J}^{v_{i}}\right) \mathrm{d} \xi_{i}} \\
{\left[\overline{\mathbf{K}}_{b}^{R}\right]_{I J}^{v_{i} w_{i}}=-\int_{0}^{1} \bar{f}_{0}^{R} \phi_{I}^{v_{i}} \frac{\mathrm{d} \phi_{J}^{w_{i}}}{\mathrm{~d} \xi_{i}} \mathrm{~d} \xi_{i}}
\end{gathered}
$$




$$
\begin{gathered}
{\left[\overline{\mathbf{K}}_{b}^{R}\right]_{I J}^{v_{i} v_{j}}=\int_{0}^{1} \bar{f}_{1}^{R} \phi_{I}^{v_{i}} \phi_{J}^{v_{j}} \mathrm{~d} \xi_{j} ; i \neq j} \\
{\left[\overline{\mathbf{K}}_{b}^{R}\right]_{I J}^{w_{i} v_{i}}=\int_{0}^{1} \bar{f}_{0}^{R} \phi_{I}^{w_{i}} \frac{\mathrm{d} \phi_{J}^{v_{i}}}{\mathrm{~d} \xi_{i}} \mathrm{~d} \xi_{i}} \\
{\left[\overline{\mathbf{K}}_{b}^{R}\right]_{I J}^{w_{i} w_{i}}=\int_{0}^{1}\left(\left(1+\chi_{3}^{R}\right) \frac{\mathrm{d}^{2} \phi_{I}^{w_{i}}}{\mathrm{~d} \xi_{i}^{2}} \frac{\mathrm{d}^{2} \phi_{J}^{w_{i}}}{\mathrm{~d} \xi_{i}^{2}}+\left(\bar{T}^{R}+\chi_{4}^{R}\right) \frac{\mathrm{d} \phi_{I}^{w_{i}}}{\mathrm{~d} \xi_{i}} \frac{\mathrm{d} \phi_{J}^{w_{j}}}{\mathrm{~d} \xi_{i}}+\bar{f}_{1}^{R} \phi_{I}^{w_{i}} \phi_{J}^{w_{i}}\right) \mathrm{d} \xi_{i},} \\
{\left[\overline{\mathbf{K}}_{b}^{R}\right]_{I J}^{w_{i} w_{j}}=-\int_{0}^{1} \bar{f}_{1}^{R} \phi_{I}^{w_{i}} \phi_{J}^{w_{j}} \mathrm{~d} \xi_{j} ; i \neq j} \\
\overline{\mathbf{v}}_{i}^{R}(\tau)=<\bar{v}_{i_{1}}^{R}(\tau), \bar{v}_{i_{2}}^{R}(\tau), \ldots, \bar{v}_{i_{N P_{i}}}^{R}(\tau)>^{\mathrm{T}} \\
\overline{\mathbf{w}}_{i}^{R}(\tau)=<\bar{w}_{i_{1}}^{R}(\tau), \bar{w}_{i_{2}}^{R}(\tau), \ldots, \bar{w}_{i_{N P_{i}}}^{R}(\tau)>^{\mathrm{T}} ; i, j=1,2 .
\end{gathered}
$$

\subsubsection{Free transverse vibration on the basis of the proposed exact model}

In order to construct the discretized exact version of the governing equations using RKPM, we follow the same procedure explained in the previous part. To this end, both sides of Eqs. (27a)-(27d) in order are premultiplied by $\delta \bar{v}_{1}^{R}, \delta \bar{w}_{1}^{R}, \delta \bar{v}_{2}^{R}$, and $\delta \bar{w}_{2}^{R}$. By integrating the resulting expressions over the dimensionless spatial domains of the CCNWs, by taking the required integration by parts, and finally, by introducing Eq. (28) to the obtained statement, one can arrive at the set of ordinary differential equations (ODEs) of the form given in Eq. (29). The mass submatrices remain unchanged; however, the following stiffness submatrices are now modified:

$$
\begin{aligned}
{\left[\overline{\mathbf{K}}_{b}^{R}\right]_{I J}^{v_{i} v_{i}}=} & \int_{0}^{1}\left(\left(1+\chi_{3}^{R}\right) \frac{\mathrm{d}^{2} \phi_{I}^{v_{i}}}{\mathrm{~d} \xi_{i}^{2}} \frac{\mathrm{d}^{2} \phi_{J}^{v_{i}}}{\mathrm{~d} \xi_{i}^{2}}+\left(\bar{T}^{R}+\chi_{4}^{R}\right) \frac{\mathrm{d} \phi_{I}^{v_{i}}}{\mathrm{~d} \xi_{i}} \frac{\mathrm{d} \phi_{J}^{v_{i}}}{\mathrm{~d} \xi_{i}}\right) \mathrm{d} \xi_{i} \\
& +\frac{\bar{f}_{1}^{R}}{2 \bar{d}} \int_{0}^{1} \int_{0}^{1} \frac{\phi_{I}^{v_{i}}\left(\xi_{i}\right) \phi_{J}^{v_{i}}\left(\xi_{i}\right)}{\left(\left(\frac{\xi_{2}-\xi_{1}}{\bar{d}}\right)^{2}+1\right)^{\frac{3}{2}} \mathrm{~d} \xi_{2} \mathrm{~d} \xi_{1},} \\
{\left[\overline{\mathbf{K}}_{b}^{R}\right]_{I J}^{v_{i} v_{j}}=} & -\frac{\bar{f}_{1}^{R}}{2 \bar{d}} \int_{0}^{1} \int_{0}^{1} \frac{\phi_{I}^{v_{i}}\left(\left(\xi_{i}-\xi_{j}\right) \frac{\mathrm{d} \phi_{J}^{v_{j}}}{\mathrm{~d} \xi_{j}}+\phi_{J}^{v_{j}}\right)}{\left(\left(\frac{\xi_{2}-\xi_{1}}{\bar{d}}\right)^{2}+1\right)^{\frac{3}{2}}} \mathrm{~d} \xi_{i} \mathrm{~d} \xi_{j} ; i \neq j,
\end{aligned}
$$




$$
\begin{aligned}
{\left[\overline{\mathbf{K}}_{b}^{R}\right]_{I J}^{w_{i} w_{i}} } & =\int_{0}^{1}\left(\left(1+\chi_{3}^{R}\right) \frac{\mathrm{d}^{2} \phi_{I}^{w_{i}}}{\mathrm{~d} \xi_{i}^{2}} \frac{\mathrm{d}^{2} \phi_{J}^{w_{i}}}{\mathrm{~d} \xi_{i}^{2}}+\left(\bar{T}^{R}+\chi_{4}^{R}\right) \frac{\mathrm{d} \phi_{I}^{w_{i}}}{\mathrm{~d} \xi_{i}} \frac{\mathrm{d} \phi_{J}^{w_{j}}}{\mathrm{~d} \xi_{i}}\right) \mathrm{d} \xi_{i}+ \\
& +\frac{\bar{f}_{1}^{R}}{2 \bar{d}} \int_{0}^{1} \int_{0}^{1} \frac{\phi_{I}^{w_{i}}\left(\xi_{i}\right) \phi_{J}^{w_{i}}\left(\xi_{i}\right)}{\left(\left(\frac{\xi_{2}-\xi_{1}}{\bar{d}}\right)^{2}+1\right)^{\frac{3}{2}} \mathrm{~d} \xi_{2} \mathrm{~d} \xi_{1}} \\
{\left[\overline{\mathbf{K}}_{b}^{R}\right]_{I J}^{w_{i} w_{j}} } & =-\frac{\bar{f}_{1}^{R}}{2 \bar{d}} \int_{0}^{1} \int_{0}^{1} \frac{\phi_{I}^{w_{i}}\left(\left(\xi_{i}-\xi_{j}\right) \frac{\mathrm{d} \phi_{J}^{w_{j}}}{\mathrm{~d} \xi_{j}}+\phi_{J}^{w_{j}}\right)}{\left(\left(\frac{\xi_{2}-\xi_{1}}{\bar{d}}\right)^{2}+1\right)^{\frac{3}{2}}} \mathrm{~d} \xi_{i} \mathrm{~d} \xi_{j} ; i \neq j
\end{aligned}
$$

where $i, j=1,2$.

\subsubsection{Enforcement of boundary conditions and frequency analysis}

For simply supported DCCNWs based on the RBT, the following boundary conditions should be enforced:

$$
\begin{aligned}
& \bar{w}_{i}^{R}(0, \tau)=\bar{w}_{i}^{R}(1, \tau)=0 ; \quad \bar{M}_{b_{y i}}^{R}(0, \tau)=\bar{M}_{b_{y i}}^{R}(1, \tau)=0 ; i=1,2, \\
& \bar{v}_{i}^{R}(0, \tau)=\bar{v}_{i}^{R}(1, \tau)=0 ; \quad \bar{M}_{b_{z i}}^{R}(0, \tau)=\bar{M}_{b_{z i}}^{R}(1, \tau)=0,
\end{aligned}
$$

where $\bar{M}_{b_{y i}}^{R}=\frac{M_{b_{y i}}^{R} l_{b}}{E_{b} I_{b}}$ and $\bar{M}_{b_{z i}}^{R}=\frac{M_{b_{z i}}^{R} l_{b}}{E_{b} I_{b}}$. To satisfy the given essential boundary conditions, corrected collocation approach (CCA) by Wagner and Liu (Wagner and Liu 2000) is

employed. Now the vectors of nodal parameter values are considered as: $<\overline{\mathbf{v}}_{i}^{R}, \overline{\mathbf{w}}_{i}^{R}>=<$ $\overline{\mathbf{v}}_{i 0}^{R}, \overline{\mathbf{w}}_{i 0}^{R}>e^{\mathrm{i} \varpi \tau} ; i=1,2$ where $\mathrm{i}=\sqrt{-1}, \overline{\mathbf{v}}_{i 0}^{R}$ and $\overline{\mathbf{w}}_{i 0}^{R}$ are the amplitude vectors associated with the $i$ th CCNW, and $\varpi$ is the dimensionless frequency. By introducing such a form to the resulting equations obtained by application of the CCA, and solving the resulting eigenvalue equations, the natural frequencies of the nanosystem are easily determined. 


\section{Development of surface energy-based formulations via TBT}

\subsection{Constitutive relations of the bulk and the surface layer}

In the context of the TBT (Timoshenko 1921, 1922), the deformation fields of the $i$ th CCNW read:

$$
\begin{aligned}
& u_{x_{i}}^{T}\left(x_{i}, y_{i}, z_{i}, t\right)=-\left(z_{i} \theta_{y_{i}}^{T}\left(x_{i}, t\right)+y_{i} \theta_{z_{i}}^{T}\left(x_{i}, t\right)\right) \\
& u_{y_{i}}^{T}\left(x_{i}, y_{i}, z_{i}, t\right)=v_{i}^{T}\left(x_{i}, t\right), u_{z_{i}}^{T}\left(x_{i}, y_{i}, z_{i}, t\right)=w_{i}^{T}\left(x_{i}, t\right),
\end{aligned}
$$

where $\theta_{y_{i}}^{T}$ and $\theta_{z_{i}}^{T}$ in order are the angles of deflection of the CCNW about the $y_{i}$ and $z_{i}$ axes. For small deformations, the only nonzero strains of the bulk as well as the surface layer are as:

$$
\epsilon_{x x_{i}}^{T}=-\left(z_{i} \frac{\partial \theta_{y_{i}}^{T}}{\partial x_{i}}+y_{i} \frac{\partial \theta_{z_{i}}^{T}}{\partial x_{i}}\right), \gamma_{x y_{i}}^{T}=\frac{\partial v_{i}^{T}}{\partial x_{i}}-\theta_{z_{i}}^{T}, \gamma_{x z_{i}}^{T}=\frac{\partial w_{i}^{T}}{\partial x_{i}}-\theta_{y_{i}}^{T} .
$$

Using the surface elasticity theory of Gurtin-Murdoch (Gurtin and Murdoch 1975, 1976, 1978), the stresses within the surface layer of the $i$ th CCNW modeled according to the TBT are stated by:

$$
\begin{aligned}
\tau_{x x_{i}}^{T} & =\tau_{0}+\left(\lambda_{0}+2 \mu_{0}\right) \frac{\partial u_{x_{i}}^{T}}{\partial x_{i}}=\tau_{0}-\left(\lambda_{0}+2 \mu_{0}\right)\left(z_{i} \frac{\partial \theta_{y_{i}}^{R}}{\partial x_{i}}+y_{i} \frac{\partial \theta_{z_{i}}^{R}}{\partial x_{i}}\right), \\
\tau_{x y_{i}}^{T} & =n_{y} \tau_{0} \frac{\partial v_{i}^{T}}{\partial x_{i}}, \quad \tau_{x z_{i}}^{T}=n_{z_{i}} \tau_{0} \frac{\partial w_{i}^{T}}{\partial x_{i}}
\end{aligned}
$$

It is assumed that the normal transverse stresses $\sigma_{y y_{i}}^{T}$ and $\sigma_{z z_{i}}^{T}$ of the bulk would vary linearly across the diameter of the CCNW such that their values at the furthest axes would be equal to their corresponding surface values. By employing Hook's law, the axial stress field within the bulk of the $i$ th CCNW is expressed by:

$$
\begin{aligned}
\sigma_{x x_{i}}^{T}= & y_{i}\left(-E_{b} \frac{\partial \theta_{z_{i}}^{T}}{\partial x_{i}}+\frac{2 \nu_{b}}{D_{0}}\left(\tau_{0} \frac{\partial^{2} v_{i}^{T}}{\partial x_{i}^{2}}-\rho_{0} \frac{\partial^{2} v_{i}^{T}}{\partial t^{2}}\right)\right)+ \\
& z_{i}\left(-E_{b} \frac{\partial \theta_{y_{i}}^{T}}{\partial x_{i}}+\frac{2 \nu_{b}}{D_{0}}\left(\tau_{0} \frac{\partial^{2} w_{i}^{T}}{\partial x_{i}^{2}}-\rho_{0} \frac{\partial^{2} w_{i}^{T}}{\partial t^{2}}\right)\right)
\end{aligned}
$$

and the shear stresses of the bulk would take the following form:

$$
\sigma_{x y_{i}}^{T}=G_{b}\left(\frac{\partial v_{i}^{T}}{\partial x_{i}}-\theta_{z_{i}}^{T}\right), \sigma_{x z_{i}}^{T}=G_{b}\left(\frac{\partial w_{i}^{T}}{\partial x_{i}}-\theta_{y_{i}}^{T}\right),
$$

where $G_{b}$ denotes the shear elastic modulus. 


\subsection{Equations of motion of magnetically affected DCCNWs using the TBT}

In the framework of the TBT, by applying the Newton's second law to an infinitesimal element of the CCNW accounting for the surface energy effect, the equations of motion of the magnetically affected DCCNWs are obtained as follows:

$$
\begin{gathered}
\frac{\partial M_{b_{z i}}^{T}}{\partial x_{i}}+\int_{\mathcal{S}} y_{i} \frac{\partial \tau_{x x_{i}}^{T}}{\partial x_{i}} \mathrm{~d} \mathcal{S}-Q_{b_{y i}}^{T}=\int_{A_{b}} \rho_{b} \frac{\partial^{2} u_{x_{i}}^{T}}{\partial t^{2}} y_{i} \mathrm{~d} A+\int_{\mathcal{S}} \rho_{0} \frac{\partial^{2} u_{x_{i}}^{T}}{\partial t^{2}} y_{i} \mathrm{~d} \mathcal{S} ; i=1,2, \\
-\frac{\partial Q_{b_{y i}}^{T}}{\partial x_{i}}-T \frac{\partial^{2} u_{y_{i}}^{T}}{\partial x_{i}^{2}}-\int_{\mathcal{S}} \frac{\partial \tau_{x y_{i}}^{T}}{\partial x_{i}} n_{y_{i}} \mathrm{~d} \mathcal{S}=f_{m_{y i}}^{T}-\int_{A_{b}} \rho_{b} \frac{\partial^{2} u_{y_{i}}^{T}}{\partial t^{2}} \mathrm{~d} A-\int_{\mathcal{S}} \rho_{0} \frac{\partial^{2} u_{y_{i}}^{T}}{\partial t^{2}} n_{y_{i}} \mathrm{~d} \mathcal{S}, \\
\quad \frac{\partial M_{b_{y i}}^{T}}{\partial x_{i}}+\int_{\mathcal{S}} z \frac{\partial \tau_{x x_{i}}^{T}}{\partial x_{i}} \mathrm{~d} \mathcal{S}-Q_{b_{z i}}^{T}=\int_{A_{b}} \rho_{b} \frac{\partial^{2} u_{x_{i}}^{T}}{\partial t^{2}} z \mathrm{~d} A+\int_{\mathcal{S}} \rho_{0} \frac{\partial^{2} u_{x_{i}}^{T}}{\partial t^{2}} z_{i} \mathrm{~d} \mathcal{S} \\
-\frac{\partial Q_{b_{z i}}^{T}}{\partial x_{i}}-T \frac{\partial^{2} w_{i}^{T}}{\partial x_{i}^{2}}-\int_{\mathcal{S}} \frac{\partial \tau_{x z_{i}}^{T}}{\partial x_{i}} n_{z_{i}} \mathrm{~d} \mathcal{S}=f_{m_{z i}}^{T}-\int_{A_{b}} \rho_{b} \frac{\partial^{2} u_{z_{i}}^{T}}{\partial t^{2}} \mathrm{~d} A-\int_{\mathcal{S}} \rho_{0} \frac{\partial^{2} u_{z_{i}}^{T}}{\partial t^{2}} n_{z_{i}} \mathrm{~d} \mathcal{S},
\end{gathered}
$$

where $Q_{b_{y i}}^{T}$ and $Q_{b_{z i}}^{T}$ are the resultant shear forces within the bulk of the $i$ th CCNW along the $y_{i}$ and $z_{i}$ axes, $M_{b_{y i}}^{T}$ and $M_{b_{z i}}^{T}$ are the resultant bending moments of the bulk about the $y_{i}$ and $z_{i}$ axes. In view of Eqs. (36) and (37), these internal forces are calculated as:

$$
\begin{gathered}
M_{b_{y i}}^{T}=\int_{A_{b}} z_{i} \sigma_{x x_{i}}^{T} \mathrm{~d} A=-E_{b} I_{b} \frac{\partial \theta_{y_{i}}^{T}}{\partial x_{i}}+\frac{2 \nu_{b} I_{b}}{D_{0}}\left(\tau_{0} \frac{\partial^{2} w_{i}^{T}}{\partial x_{i}^{2}}-\rho_{0} \frac{\partial^{2} w_{i}^{T}}{\partial t^{2}}\right), \\
M_{b_{z i}}^{T}=\int_{A_{b}} y_{i} \sigma_{x x_{i}}^{T} \mathrm{~d} A=-E_{b} I_{b} \frac{\partial \theta_{z_{i}}^{T}}{\partial x_{i}}+\frac{2 \nu_{b} I_{b}}{D_{0}}\left(\tau_{0} \frac{\partial^{2} v_{i}^{T}}{\partial x_{i}^{2}}-\rho_{0} \frac{\partial^{2} v_{i}^{T}}{\partial t^{2}}\right), \\
<Q_{b_{y i}}^{T}, Q_{b_{z i}}^{T}>=k_{s} \int_{A_{b}}<\sigma_{x y_{i}}^{T}, \sigma_{x z_{i}}^{T}>\mathrm{d} A=k_{s} G_{b} A_{b}<\frac{\partial v_{i}^{T}}{\partial x_{i}}-\theta_{z_{i}}^{T}, \frac{\partial w_{i}^{T}}{\partial x_{i}}-\theta_{y_{i}}^{T}>,
\end{gathered}
$$

where $k_{s}$ is the shear correction factor.

\subsubsection{An approximate version of the governing equations based on the TBT}

By substituting Eqs. (10a) and (10b), (33), (35), (39a), and (39b) into Eqs. (38a)-(38d), the governing equations of the magnetically affected DCCNWs in terms of displacement 
fields of the TBT are obtained:

$$
\begin{aligned}
& \left(\rho_{b} I_{b}+\rho_{0} I_{0}^{*}\right) \frac{\partial^{2} \theta_{z_{1}}^{T}}{\partial t^{2}}-\frac{2 \nu_{b} I_{b} \rho_{0}}{D_{0}} \frac{\partial^{3} v_{1}^{T}}{\partial t^{2} \partial x_{1}}+\frac{2 \nu_{b} I_{b} \tau_{0}}{D_{0}} \frac{\partial^{3} v_{1}^{T}}{\partial x_{1}^{3}} \\
& -k_{s} G_{b} A_{b}\left(\frac{\partial v_{1}^{T}}{\partial x_{1}}-\theta_{z_{1}}^{T}\right)-\left(E_{b} I_{b}+\left(\lambda_{0}+2 \mu_{0}\right) I_{0}^{*}\right) \frac{\partial^{2} \theta_{z_{1}}^{T}}{\partial x_{1}^{2}}=0, \\
& \left(\rho_{b} A_{b}+\rho_{0} S_{0}^{*}\right) \frac{\partial^{2} v_{1}^{T}}{\partial t^{2}}-k_{s} G_{b} A_{b}\left(\frac{\partial^{2} v_{1}^{T}}{\partial x_{1}^{2}}-\frac{\partial \theta_{z_{1}}^{T}}{\partial x_{1}}\right) \\
& -T \frac{\partial^{2} v_{1}^{T}}{\partial x_{1}^{2}}-B_{0} I_{0} \frac{\partial w_{1}^{T}}{\partial x_{1}}+\frac{\mu_{0} I_{0}^{2}}{2 \pi d^{2}}\left(v_{2}^{T}-v_{1}^{T}\right)=0, \\
& \left(\rho_{b} I_{b}+\rho_{0} I_{0}^{*}\right) \frac{\partial^{2} \theta_{y_{1}}^{T}}{\partial t^{2}}-\frac{2 \nu_{b} I_{b} \rho_{0}}{D_{0}} \frac{\partial^{3} w_{1}^{T}}{\partial t^{2} \partial x}+\frac{2 \nu_{b} I_{b} \tau_{0}}{D_{0}} \frac{\partial^{3} w_{1}^{T}}{\partial x^{3}} \\
& -k_{s} G_{b} A_{b}\left(\frac{\partial w_{1}^{T}}{\partial x_{1}}-\theta_{y_{1}}^{T}\right)-\left(E_{b} I_{b}+\left(\lambda_{0}+2 \mu_{0}\right) I_{0}^{*}\right) \frac{\partial^{2} \theta_{y_{1}}^{T}}{\partial x_{1}^{2}}=0, \\
& \left(\rho_{b} A_{b}+\rho_{0} S_{0}^{*}\right) \frac{\partial^{2} w_{1}^{T}}{\partial t^{2}}-k_{s} G_{b} A_{b}\left(\frac{\partial^{2} w_{1}^{T}}{\partial x_{1}^{2}}-\frac{\partial \theta_{y_{1}}^{T}}{\partial x_{1}}\right) \\
& -T \frac{\partial^{2} w_{1}^{T}}{\partial x_{1}^{2}}+B_{0} I_{0} \frac{\partial v_{1}^{T}}{\partial x}-\frac{\mu_{0} I_{0}^{2}}{2 \pi d^{2}}\left(w_{2}^{T}-w_{1}^{T}\right)=0, \\
& \left(\rho_{b} I_{b}+\rho_{0} I_{0}^{*}\right) \frac{\partial^{2} \theta_{z_{2}}^{T}}{\partial t^{2}}-\frac{2 \nu_{b} I_{b} \rho_{0}}{D_{0}} \frac{\partial^{3} v_{2}^{T}}{\partial t^{2} \partial x_{2}}+\frac{2 \nu_{b} I_{b} \tau_{0}}{D_{0}} \frac{\partial^{3} v_{2}^{T}}{\partial x_{2}^{3}} \\
& -k_{s} G_{b} A_{b}\left(\frac{\partial v_{2}^{T}}{\partial x_{2}}-\theta_{z_{2}}^{T}\right)-\left(E_{b} I_{b}+\left(\lambda_{0}+2 \mu_{0}\right) I_{0}^{*}\right) \frac{\partial^{2} \theta_{z_{2}}^{T}}{\partial x_{2}^{2}}=0 \\
& \left(\rho_{b} A_{b}+\rho_{0} S_{0}^{*}\right) \frac{\partial^{2} v_{2}^{T}}{\partial t^{2}}-k_{s} G_{b} A_{b}\left(\frac{\partial^{2} v_{2}^{T}}{\partial x_{2}^{2}}-\frac{\partial \theta_{z_{2}}^{T}}{\partial x_{2}}\right) \\
& -T \frac{\partial^{2} v_{2}^{T}}{\partial x_{2}^{2}}-B_{0} I_{0} \frac{\partial w_{2}^{T}}{\partial x_{2}}-\frac{\mu_{0} I_{0}^{2}}{2 \pi d^{2}}\left(v_{2}^{T}-v_{1}^{T}\right)=0, \\
& \left(\rho_{b} I_{b}+\rho_{0} I_{0}^{*}\right) \frac{\partial^{2} \theta_{y_{2}}^{T}}{\partial t^{2}}-\frac{2 \nu_{b} I_{b} \rho_{0}}{D_{0}} \frac{\partial^{3} w_{2}^{T}}{\partial t^{2} \partial x_{2}}+\frac{2 \nu_{b} I_{b} \tau_{0}}{D_{0}} \frac{\partial^{3} w_{2}^{T}}{\partial x_{2}^{3}} \\
& -k_{s} G_{b} A_{b}\left(\frac{\partial w_{2}^{T}}{\partial x_{2}}-\theta_{y_{2}}^{T}\right)-\left(E_{b} I_{b}+\left(\lambda_{0}+2 \mu_{0}\right) I_{0}^{*}\right) \frac{\partial^{2} \theta_{y_{2}}^{T}}{\partial x_{2}^{2}}=0,
\end{aligned}
$$




$$
\begin{aligned}
& \left(\rho_{b} A_{b}+\rho_{0} S_{0}^{*}\right) \frac{\partial^{2} w_{2}^{T}}{\partial t^{2}}-k_{s} G_{b} A_{b}\left(\frac{\partial^{2} w_{2}^{T}}{\partial x_{2}^{2}}-\frac{\partial \theta_{y_{2}}^{T}}{\partial x_{2}}\right) \\
& -T \frac{\partial^{2} w_{2}^{T}}{\partial x_{2}^{2}}+B_{0} I_{0} \frac{\partial v_{2}^{T}}{\partial x_{2}}+\frac{\mu_{0} I_{0}^{2}}{2 \pi d^{2}}\left(w_{2}^{T}-w_{1}^{T}\right)=0 .
\end{aligned}
$$

By defining the following dimensionless quantities,

$$
\begin{aligned}
& \bar{v}_{i}^{T}=\frac{v_{i}^{T}}{l_{b}}, \bar{w}_{i}^{T}=\frac{w_{i}^{T}}{l_{b}}, \bar{\theta}_{y_{i}}^{T}=\theta_{y_{i}}^{T}, \bar{\theta}_{z_{i}}^{T}=\theta_{z_{i}}^{T}, \tau=\frac{t}{l_{b}} \sqrt{\frac{G_{b}}{\rho_{b}}}, \eta=\frac{E_{b} I_{b}}{k_{s} G_{b} A_{b} l_{b}^{2}}, \\
& \bar{f}_{0}^{T}=\frac{B_{0} I_{0} l_{b}}{k_{s} G_{b} A_{b}}, \bar{f}_{1}^{T}=\frac{\mu_{0} I_{0}^{2} l_{b}^{2}}{2 \pi d^{2} k_{s} G_{b} A_{b}}, \bar{T}^{T}=\frac{T}{k_{s} G_{b} A_{b}}, \chi_{1}^{T}=\frac{\rho_{0} S_{0}^{*}}{\rho_{b} A_{b}}, \chi_{2}^{T}=\frac{\rho_{0} I_{0}^{*}}{\rho_{b} I_{b}}, \\
& \chi_{3}^{T}=\frac{2 \nu_{b} \rho_{0}}{\rho_{b} D_{0}}, \chi_{4}^{T}=\frac{2 \nu_{b} I_{b} \tau_{0}}{k_{s} G_{b} A_{b} l_{b}^{2} D_{0}}, \chi_{5}^{T}=\frac{\left(\lambda_{0}+2 \mu_{0}\right) I_{0}^{*}}{k_{s} G_{b} A b_{b} l_{b}^{2}}, \chi_{6}^{T}=\frac{\tau_{0} S_{0}^{*}}{k_{s} G_{b} A_{b}},
\end{aligned}
$$

the dimensionless equations of motion of the DCCNWs acted upon by a longitudinal magnetic field based on the TBT and the given approximate magnetic forces are readily derived:

$$
\begin{aligned}
& \lambda^{-2}\left(1+\chi_{2}^{T}\right) \frac{\partial^{2} \bar{\theta}_{z_{1}}^{T}}{\partial \tau^{2}}-\lambda^{-2} \chi_{3}^{T} \frac{\partial^{3} \bar{v}_{1}^{T}}{\partial \tau^{2} \partial \xi_{1}}+\chi_{4}^{T} \frac{\partial^{3} \bar{v}_{1}^{T}}{\partial \xi_{1}^{3}}-\left(\eta+\chi_{5}^{T}\right) \frac{\partial^{2} \bar{\theta}_{z_{1}}^{T}}{\partial \xi_{1}^{2}}-\frac{\partial \bar{v}_{1}^{T}}{\partial \xi_{1}}+\bar{\theta}_{z_{1}}^{T}=0, \\
& \left(1+\chi_{1}^{T}\right) \frac{\partial^{2} \bar{v}_{1}^{T}}{\partial \tau^{2}}+\frac{\partial \bar{\theta}_{z_{1}}^{T}}{\partial \xi_{1}}-\left(1+\bar{T}^{T}+\chi_{6}^{T}\right) \frac{\partial^{2} \bar{v}_{1}^{T}}{\partial \xi_{1}^{2}}-\bar{f}_{0}^{T} \frac{\partial \bar{w}_{1}^{T}}{\partial \xi_{1}}+\bar{f}_{1}^{T}\left(\bar{v}_{2}^{T}-\bar{v}_{1}^{T}\right)=0 \\
& \lambda^{-2}\left(1+\chi_{2}^{T}\right) \frac{\partial^{2} \bar{\theta}_{y_{1}}^{T}}{\partial \tau^{2}}-\lambda^{-2} \chi_{3}^{T} \frac{\partial^{3} \bar{w}_{1}^{T}}{\partial \tau^{2} \partial \xi_{1}}+\chi_{4}^{T} \frac{\partial^{3} \bar{w}_{1}^{T}}{\partial \xi_{1}^{3}}-\left(\eta+\chi_{5}^{T}\right) \frac{\partial^{2} \bar{\theta}_{y_{1}}^{T}}{\partial \xi_{1}^{2}}-\frac{\partial \bar{w}_{1}^{T}}{\partial \xi_{1}}+\bar{\theta}_{y_{1}}^{T}=0 \\
& \left(1+\chi_{1}^{T}\right) \frac{\partial^{2} \bar{w}_{1}^{T}}{\partial \tau^{2}}+\frac{\partial \bar{\theta}_{y_{1}}^{T}}{\partial \xi_{1}}-\left(1+\bar{T}^{T}+\chi_{6}^{T}\right) \frac{\partial^{2} \bar{w}_{1}^{T}}{\partial \xi_{1}^{2}}+\bar{f}_{0}^{T} \frac{\partial \bar{v}_{1}^{T}}{\partial \xi_{1}}-\bar{f}_{1}^{T}\left(\bar{w}_{2}^{T}-\bar{w}_{1}^{T}\right)=0 \\
& \lambda^{-2}\left(1+\chi_{2}^{T}\right) \frac{\partial^{2} \bar{\theta}_{z_{2}}^{T}}{\partial \tau^{2}}-\lambda^{-2} \chi_{3}^{T} \frac{\partial^{3} \bar{v}_{2}^{T}}{\partial \tau^{2} \partial \xi_{2}}+\chi_{4}^{T} \frac{\partial^{3} \bar{v}_{2}^{T}}{\partial \xi_{2}^{3}}-\left(\eta+\chi_{5}^{T}\right) \frac{\partial^{2} \bar{\theta}_{z_{2}}^{T}}{\partial \xi_{2}^{2}}-\frac{\partial \bar{v}_{2}^{T}}{\partial \xi_{2}}+\bar{\theta}_{z_{2}}^{T}=0, \\
& \left(1+\chi_{1}^{T}\right) \frac{\partial^{2} \bar{v}_{2}^{T}}{\partial \tau^{2}}+\frac{\partial \bar{\theta}_{z_{2}}^{T}}{\partial \xi_{2}}-\left(1+\bar{T}^{T}+\chi_{6}^{T}\right) \frac{\partial^{2} \bar{v}_{2}^{T}}{\partial \xi_{2}^{2}}-\bar{f}_{0}^{T} \frac{\partial \bar{w}_{2}^{T}}{\partial \xi_{2}}-\bar{f}_{1}^{T}\left(\bar{v}_{2}^{T}-\bar{v}_{1}^{T}\right)=0
\end{aligned}
$$




$$
\begin{gathered}
\lambda^{-2}\left(1+\chi_{2}^{T}\right) \frac{\partial^{2} \bar{\theta}_{y_{2}}^{T}}{\partial \tau^{2}}-\lambda^{-2} \chi_{3}^{T} \frac{\partial^{3} \bar{w}_{2}^{T}}{\partial \tau^{2} \partial \xi_{2}}+\chi_{4}^{T} \frac{\partial^{3} \bar{w}_{2}^{T}}{\partial \xi_{2}^{3}}-\left(\eta+\chi_{5}^{T}\right) \frac{\partial^{2} \bar{\theta}_{y_{2}}^{T}}{\partial \xi_{2}^{2}}-\frac{\partial \bar{w}_{2}^{T}}{\partial \xi_{2}}+\bar{\theta}_{y_{2}}^{T}=0 \\
\left(1+\chi_{1}^{T}\right) \frac{\partial^{2} \bar{w}_{2}^{T}}{\partial \tau^{2}}+\frac{\partial \bar{\theta}_{y_{2}}^{T}}{\partial \xi_{2}}-\left(1+\bar{T}^{T}+\chi_{6}^{T}\right) \frac{\partial^{2} \bar{w}_{2}^{T}}{\partial \xi_{2}^{2}}+\bar{f}_{0}^{T} \frac{\partial \bar{v}_{2}^{T}}{\partial \xi_{2}}+\bar{f}_{1}^{T}\left(\bar{w}_{2}^{T}-\bar{w}_{1}^{T}\right)=0
\end{gathered}
$$

By excluding the surface energy effect (i.e., $\left.\chi_{i}^{T}=0 ; i=1,2, \ldots, 6\right)$, Eqs. (42a)-(42h) reduce to the equations of motion of doubly parallel macrowires immersed in a longitudinal magnetic field based on the TBT. Eqs. (42a)-(42h) represent eight coupled second-order PDEs that finding an exact solution to them is a cumbersome job. As a result, development of an efficient numerical methodology in analyzing these equations would be of great importance.

\subsubsection{An exact version of the governing equations based on the TBT}

In order to extract exact governing equations, we use the exact version of the mutually exerted interwire magnetic forces. To this end, Eqs. (14a) and (14b) plus to Eqs. (33), (35), (39a), and (39b) are substituted into Eqs. (38a)-(38d). Therefore,

$$
\begin{gathered}
\left(\rho_{b} I_{b}+\rho_{0} I_{0}^{*}\right) \frac{\partial^{2} \theta_{z_{1}}^{T}}{\partial t^{2}}-\frac{2 \nu_{b} I_{b} \rho_{0}}{D_{0}} \frac{\partial^{3} v_{1}^{T}}{\partial t^{2} \partial x_{1}}+\frac{2 \nu_{b} I_{b} \tau_{0}}{D_{0}} \frac{\partial^{3} v_{1}^{T}}{\partial x_{1}^{3}} \\
-k_{s} G_{b} A_{b}\left(\frac{\partial v_{1}^{T}}{\partial x_{1}}-\theta_{z_{1}}^{T}\right)-\left(E_{b} I_{b}+\left(\lambda_{0}+2 \mu_{0}\right) I_{0}^{*}\right) \frac{\partial^{2} \theta_{z_{1}}^{T}}{\partial x_{1}^{2}}=0 \\
\left(\rho_{b} A_{b}+\rho_{0} S_{0}^{*}\right) \frac{\partial^{2} v_{1}^{T}}{\partial t^{2}}-k_{s} G_{b} A_{b}\left(\frac{\partial^{2} v_{1}^{T}}{\partial x_{1}^{2}}-\frac{\partial \theta_{z_{1}}^{T}}{\partial x_{1}}\right) \\
-T \frac{\partial^{2} v_{1}^{T}}{\partial x_{1}^{2}}-B_{0} I_{0} \frac{\partial w_{1}^{T}}{\partial x_{1}}+\frac{\mu_{0} I_{0}^{2}}{4 \pi} \int_{0}^{l_{b}} \frac{\left(x_{2}-x_{1}\right) \frac{\partial v_{2}^{T}}{\partial x_{2}}-\left(v_{2}^{T}-v_{1}^{T}\right)}{\left(\left(x_{2}-x_{1}\right)^{2}+d^{2}\right)^{\frac{3}{2}}} \mathrm{~d} x_{2}=0 \\
\left(\rho_{b} I_{b}+\rho_{0} I_{0}^{*}\right) \frac{\partial^{2} \theta_{y_{1}}^{T}}{\partial t^{2}}-\frac{2 \nu_{b} I_{b} \rho_{0}}{D_{0}} \frac{\partial^{3} w_{1}^{T}}{\partial t^{2} \partial x_{1}}+\frac{2 \nu_{b} I_{b} \tau_{0}}{D_{0}} \frac{\partial^{3} w_{1}^{T}}{\partial x_{1}^{3}} \\
-k_{s} G_{b} A_{b}\left(\frac{\partial w_{1}^{T}}{\partial x_{1}}-\theta_{y_{1}}^{T}\right)-\left(E_{b} I_{b}+\left(\lambda_{0}+2 \mu_{0}\right) I_{0}^{*}\right) \frac{\partial^{2} \theta_{y_{1}}^{T}}{\partial x_{1}^{2}}=0 \\
\left(\rho_{b} A_{b}+\rho_{0} S_{0}^{*}\right) \frac{\partial^{2} w_{1}^{T}}{\partial t^{2}}-k_{s} G_{b} A_{b}\left(\frac{\partial^{2} w_{1}^{T}}{\partial x_{1}^{2}}-\frac{\partial \theta_{y_{1}}^{T}}{\partial x_{1}}\right) \\
-T \frac{\partial^{2} w_{1}^{T}}{\partial x_{1}^{2}}+B_{0} I_{0} \frac{\partial v_{1}^{T}}{\partial x_{1}}+\frac{\mu_{0} I_{0}^{2}}{4 \pi} \int_{0}^{l_{b}} \frac{\left(x_{2}-x_{1}\right) \frac{\partial w_{2}^{T}}{\partial x_{2}}-\left(w_{2}^{T}-w_{1}^{T}\right)}{\left.\left(x_{2}-x_{1}\right)^{2}+d^{2}\right)^{\frac{3}{2}}} \mathrm{~d} x_{2}=0, \\
26
\end{gathered}
$$




$$
\begin{gathered}
\left(\rho_{b} I_{b}+\rho_{0} I_{0}^{*}\right) \frac{\partial^{2} \theta_{z_{2}}^{T}}{\partial t^{2}}-\frac{2 \nu_{b} I_{b} \rho_{0}}{D_{0}} \frac{\partial^{3} v_{2}^{T}}{\partial t^{2} \partial x_{2}}+\frac{2 \nu_{b} I_{b} \tau_{0}}{D_{0}} \frac{\partial^{3} v_{2}^{T}}{\partial x_{2}^{3}} \\
-k_{s} G_{b} A_{b}\left(\frac{\partial v_{2}^{T}}{\partial x_{2}}-\theta_{z_{2}}^{T}\right)-\left(E_{b} I_{b}+\left(\lambda_{0}+2 \mu_{0}\right) I_{0}^{*}\right) \frac{\partial^{2} \theta_{z_{2}}^{T}}{\partial x_{2}^{2}}=0 \\
\left(\rho_{b} A_{b}+\rho_{0} S_{0}^{*}\right) \frac{\partial^{2} v_{2}^{T}}{\partial t^{2}}-k_{s} G_{b} A_{b}\left(\frac{\partial^{2} v_{2}^{T}}{\partial x_{2}^{2}}-\frac{\partial \theta_{z_{2}}^{T}}{\partial x_{2}}\right) \\
-T \frac{\partial^{2} v_{2}^{T}}{\partial x_{2}^{2}}-B_{0} I_{0} \frac{\partial w_{2}^{T}}{\partial x_{2}}-\frac{\mu_{0} I_{0}^{2}}{4 \pi} \int_{0}^{l_{b}} \frac{\left(x_{2}-x_{1}\right) \frac{\partial v_{1}^{T}}{\partial x_{1}}-\left(v_{2}^{T}-v_{1}^{T}\right)}{\left(\left(x_{2}-x_{1}\right)^{2}+d^{2}\right)^{\frac{3}{2}}} \mathrm{~d} x_{1}=0 \\
\left(\rho_{b} I_{b}+\rho_{0} I_{0}^{*}\right) \frac{\partial^{2} \theta_{y_{2}}^{T}}{\partial t^{2}}-\frac{2 \nu_{b} I_{b} \rho_{0}}{D_{0}} \frac{\partial^{3} w_{2}^{T}}{\partial t^{2} \partial x_{2}}+\frac{2 \nu_{b} I_{b} \tau_{0}}{D_{0}} \frac{\partial^{3} w_{2}^{T}}{\partial x_{2}^{3}} \\
-k_{s} G_{b} A_{b}\left(\frac{\partial w_{2}^{T}}{\partial x_{2}}-\theta_{y_{2}}^{T}\right)-\left(E_{b} I_{b}+\left(\lambda_{0}+2 \mu_{0}\right) I_{0}^{*}\right) \frac{\partial^{2} \theta_{y_{2}}^{T}}{\partial x_{2}^{2}}=0 \\
\left(\rho_{b} A_{b}+\rho_{0} S_{0}^{*}\right) \frac{\partial^{2} w_{2}^{T}}{\partial t^{2}}-k_{s} G_{b} A_{b}\left(\frac{\partial^{2} w_{2}^{T}}{\partial x_{2}^{2}}-\frac{\partial \theta_{y_{2}}^{T}}{\partial x_{2}}\right) \\
-T \frac{\partial^{2} w_{2}^{T}}{\partial x_{2}^{2}}+B_{0} I_{0} \frac{\partial v_{2}^{T}}{\partial x_{2}}-\frac{\mu_{0} I_{0}^{2}}{4 \pi} \int_{0}^{l_{b}} \frac{\left(x_{2}-x_{1}\right) \frac{\partial w_{1}^{T}}{\partial x_{1}}-\left(w_{2}^{T}-w_{1}^{T}\right)}{\left.\left(x_{2}-x_{1}\right)^{2}+d^{2}\right)^{\frac{3}{2}}} \mathrm{~d} x_{1}=0
\end{gathered}
$$

By introducing Eq. (41) to Eqs. (43a)-(43h), the exact dimensionless transverse equations of motion of the DCCNWs acted upon by a longitudinal magnetic field based on the TBT are extracted as:

$$
\begin{aligned}
& \lambda^{-2}\left(1+\chi_{2}^{T}\right) \frac{\partial^{2} \bar{\theta}_{z_{1}}^{T}}{\partial \tau^{2}}-\lambda^{-2} \chi_{3}^{T} \frac{\partial^{3} \bar{v}_{1}^{T}}{\partial \tau^{2} \partial \xi_{1}}+\chi_{4}^{T} \frac{\partial^{3} \bar{v}_{1}^{T}}{\partial \xi_{1}^{3}} \\
& -\left(\eta+\chi_{5}^{T}\right) \frac{\partial^{2} \bar{\theta}_{z_{1}}^{T}}{\partial \xi_{1}^{2}}-\frac{\partial \bar{v}_{1}^{T}}{\partial \xi_{1}}+\bar{\theta}_{z_{1}}^{T}=0, \\
& \left(1+\chi_{1}^{T}\right) \frac{\partial^{2} \bar{v}_{1}^{T}}{\partial \tau^{2}}+\frac{\partial \bar{\theta}_{z_{1}}^{T}}{\partial \xi_{1}}-\left(1+\bar{T}^{T}+\chi_{6}^{T}\right) \frac{\partial^{2} \bar{v}_{1}^{T}}{\partial \xi_{1}^{2}} \\
& -\bar{f}_{0}^{T} \frac{\bar{w}_{1}^{T}}{\partial \xi_{1}}+\frac{\bar{f}_{1}^{T}}{2 \bar{d}} \int_{0}^{1} \frac{\left(\xi_{2}-\xi_{1}\right) \frac{\partial \bar{v}_{2}^{T}}{\partial \xi_{2}}-\left(\bar{v}_{2}^{T}-\bar{v}_{1}^{T}\right)}{\left(\left(\frac{\xi_{2}-\xi_{1}}{\bar{d}}\right)^{2}+1\right)^{\frac{3}{2}}} \mathrm{~d} \xi_{2}=0,
\end{aligned}
$$




$$
\begin{aligned}
& \lambda^{-2}\left(1+\chi_{2}^{T}\right) \frac{\partial^{2} \bar{\theta}_{y_{1}}^{T}}{\partial \tau^{2}}-\lambda^{-2} \chi_{3}^{T} \frac{\partial^{3} \bar{w}_{1}^{T}}{\partial \tau^{2} \partial \xi_{1}}+\chi_{4}^{T} \frac{\partial^{3} \bar{w}_{1}^{T}}{\partial \xi_{1}^{3}} \\
& -\left(\eta+\chi_{5}^{T}\right) \frac{\partial^{2} \bar{\theta}_{y_{1}}^{T}}{\partial \xi_{1}^{2}}-\frac{\partial \bar{w}_{1}^{T}}{\partial \xi_{1}}+\bar{\theta}_{y_{1}}^{T}=0, \\
& \left(1+\chi_{1}^{T}\right) \frac{\partial^{2} \bar{w}_{1}^{T}}{\partial \tau^{2}}+\frac{\partial \bar{\theta}_{y_{1}}^{T}}{\partial \xi_{1}}-\left(1+\bar{T}^{T}+\chi_{6}^{T}\right) \frac{\partial^{2} \bar{w}_{1}^{T}}{\partial \xi_{1}^{2}} \\
& +\bar{f}_{0}^{T} \frac{\bar{v}_{1}^{T}}{\partial \xi_{1}}+\frac{\bar{f}_{1}^{T}}{2 \bar{d}} \int_{0}^{1} \frac{\left(\xi_{2}-\xi_{1}\right) \frac{\partial \bar{w}_{2}^{T}}{\partial \xi_{2}}-\left(\bar{w}_{2}^{T}-\bar{w}_{1}^{T}\right)}{\left(\left(\frac{\xi_{2}-\xi_{1}}{\bar{d}}\right)^{2}+1\right)^{\frac{3}{2}}} \mathrm{~d} \xi_{2}=0, \\
& \lambda^{-2}\left(1+\chi_{2}^{T}\right) \frac{\partial^{2} \bar{\theta}_{z_{2}}^{T}}{\partial \tau^{2}}-\lambda^{-2} \chi_{3}^{T} \frac{\partial^{3} \bar{v}_{2}^{T}}{\partial \tau^{2} \partial \xi_{2}}+\chi_{4}^{T} \frac{\partial^{3} \bar{v}_{2}^{T}}{\partial \xi_{2}^{3}} \\
& -\left(\eta+\chi_{5}^{T}\right) \frac{\partial^{2} \bar{\theta}_{z_{2}}^{T}}{\partial \xi_{2}^{2}}-\frac{\partial \bar{v}_{2}^{T}}{\partial \xi_{2}}+\bar{\theta}_{z_{2}}^{T}=0, \\
& \left(1+\chi_{1}^{T}\right) \frac{\partial^{2} \bar{v}_{2}^{T}}{\partial \tau^{2}}+\frac{\partial \bar{\theta}_{z_{2}}^{T}}{\partial \xi_{2}}-\left(1+\bar{T}^{T}+\chi_{6}^{T}\right) \frac{\partial^{2} \bar{v}_{2}^{T}}{\partial \xi_{2}^{2}} \\
& -\bar{f}_{0}^{T} \frac{\bar{w}_{2}^{T}}{\partial \xi_{2}}-\frac{\bar{f}_{1}^{T}}{2 \bar{d}} \int_{0}^{1} \frac{\left(\xi_{2}-\xi_{1}\right) \frac{\partial \bar{v}_{1}^{T}}{\partial \xi_{1}}-\left(\bar{v}_{2}^{T}-\bar{v}_{1}^{T}\right)}{\left(\left(\frac{\xi_{2}-\xi_{1}}{\bar{d}}\right)^{2}+1\right)^{\frac{3}{2}}} \mathrm{~d} \xi_{1}=0, \\
& \lambda^{-2}\left(1+\chi_{2}^{T}\right) \frac{\partial^{2} \bar{\theta}_{y_{2}}^{T}}{\partial \tau^{2}}-\lambda^{-2} \chi_{3}^{T} \frac{\partial^{3} \bar{w}_{2}^{T}}{\partial \tau^{2} \partial \xi_{2}}+\chi_{4}^{T} \frac{\partial^{3} \bar{w}_{2}^{T}}{\partial \xi_{2}^{3}} \\
& -\left(\eta+\chi_{5}^{T}\right) \frac{\partial^{2} \bar{\theta}_{y_{2}}^{T}}{\partial \xi_{2}^{2}}-\frac{\partial \bar{w}_{2}^{T}}{\partial \xi_{2}}+\bar{\theta}_{y_{2}}^{T}=0, \\
& \left(1+\chi_{1}^{T}\right) \frac{\partial^{2} \bar{w}_{2}^{T}}{\partial \tau^{2}}+\frac{\partial \bar{\theta}_{y_{2}}^{T}}{\partial \xi_{2}}-\left(1+\bar{T}^{T}+\chi_{6}^{T}\right) \frac{\partial^{2} \bar{w}_{2}^{T}}{\partial \xi_{2}^{2}} \\
& +\bar{f}_{0}^{T} \frac{\bar{v}_{2}^{T}}{\partial \xi_{2}}-\frac{\bar{f}_{1}^{T}}{2 \bar{d}} \int_{0}^{1} \frac{\left(\xi_{2}-\xi_{1}\right) \frac{\partial \bar{w}_{1}^{T}}{\partial \xi_{1}}-\left(\bar{w}_{2}^{T}-\bar{w}_{1}^{T}\right)}{\left(\left(\frac{\xi_{2}-\xi_{1}}{\bar{d}}\right)^{2}+1\right)^{\frac{3}{2}}} \mathrm{~d} \xi_{1}=0 .
\end{aligned}
$$


Eqs. (44a)-(44h) furnish us regarding the exact version of the dimensionless equations of motion of DCCNWs in the presence of a longitudinal magnetic field. In fact, these are eight coupled IPDEs that should be appropriately solved. In the following part, an efficient methodology is suggested for free dynamic analysis of both approximate and exact versions of the governing equations.

\subsection{Vibration of the magnetically affected nanosystem based on the TBT via RKPM}

\subsubsection{Free transverse vibration on the basis of the proposed approximate model}

To analyze the problem using RKPM, the deformation fields of the magnetically affected DCCNWs on the basis of the TBT are discretized as follows:

$$
\begin{aligned}
& \bar{v}_{i}^{T}\left(\xi_{i}, \tau\right)=\sum_{I=1}^{N P_{i}} \phi_{I}^{v_{i}}\left(\xi_{i}\right) \bar{v}_{i_{I}}^{T}(\tau), \quad \bar{\theta}_{z_{i}}^{T}\left(\xi_{i}, \tau\right)=\sum_{I=1}^{N P_{i}} \phi_{I}^{\theta_{z_{i}}}\left(\xi_{i}\right) \bar{\theta}_{z_{i_{I}}}^{T}(\tau) ; i=1,2, \\
& \bar{w}_{i}^{T}\left(\xi_{i}, \tau\right)=\sum_{I=1}^{N P_{i}} \phi_{I}^{w_{i}}\left(\xi_{i}\right) \bar{w}_{i_{I}}^{T}(\tau), \quad \bar{\theta}_{y_{i}}^{T}\left(\xi_{i}, \tau\right)=\sum_{I=1}^{N P_{i}} \phi_{I}^{\theta_{y_{i}}}\left(\xi_{i}\right) \bar{\theta}_{y_{i_{I}}}^{T}(\tau),
\end{aligned}
$$

where $\phi_{I}^{v_{i}}, \phi_{I}^{w_{i}}, \phi_{I}^{\theta_{z_{i}}}$, and $\phi_{I}^{\theta_{y_{i}}}$ are the RKPM's shape functions of the Ith particle associated with the deformation fields $v_{i}^{T}, w_{i}^{T}, \theta_{z_{i}}^{T}$, and $\theta_{y_{i}}^{T}$, respectively, and $\bar{v}_{i_{I}}^{T}, \bar{w}_{i_{I}}^{T}, \bar{\theta}_{z_{i_{I}}}^{T}$, and $\bar{\theta}_{y_{i_{I}}}^{T}$ are their corresponding dimensionless nodal parameter values. To construct the weak form, both sides of Eqs. (42a)-(42h) are premultiplied by $\delta \bar{\theta}_{z_{1}}^{T}, \delta \bar{v}_{1}^{T}, \delta \bar{\theta}_{y_{1}}^{T}, \delta \bar{w}_{1}^{T}, \delta \bar{\theta}_{z_{2}}^{T}, \delta \bar{v}_{2}^{T}, \delta \bar{\theta}_{y_{2}}^{T}$, and $\delta \bar{w}_{2}^{T}$, respectively, and then the resulting relations are integrated over the dimensionless longitudinal domains of the CCNWs. By taking the required integration by parts, the 
following set of ordinary differential equations are obtainable:

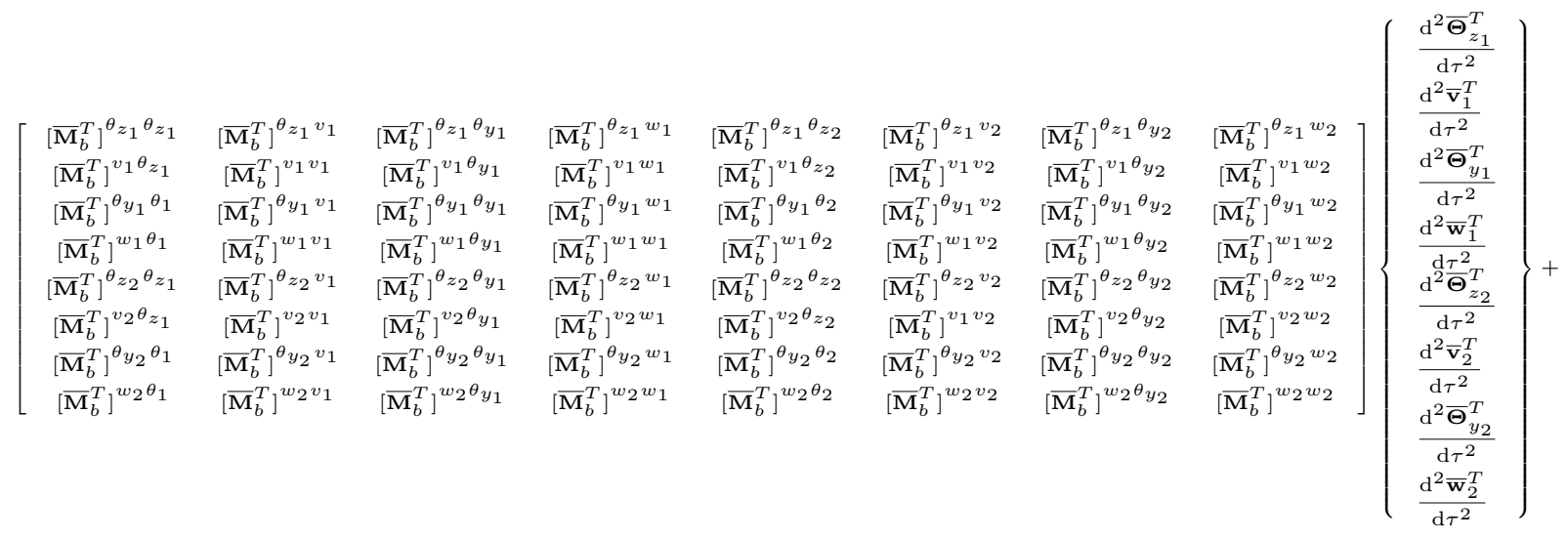

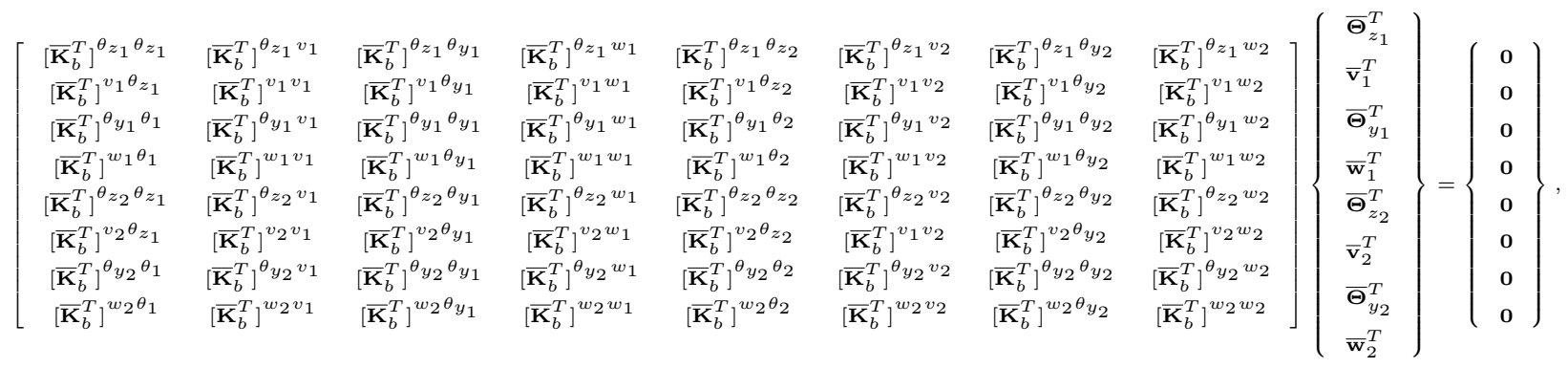

where the vectors of the dimensionless nodal parameter values as well as the nonzero dimensionless mass and stiffness submatrices are given by:

$$
\begin{gathered}
{\left[\overline{\mathbf{M}}_{b}^{T}\right]_{I J}^{\theta_{z_{i}} \theta_{z_{i}}}=\int_{0}^{1} \lambda^{-2}\left(1+\chi_{2}^{T}\right) \phi_{I}^{\theta_{z_{i}}} \phi_{J}^{\theta_{z_{i}}} \mathrm{~d} \xi_{i},} \\
{\left[\overline{\mathbf{M}}_{b}^{T}\right]_{I J}^{\theta_{z_{i}} v_{i}}=\int_{0}^{1} \lambda^{-2} \chi_{3}^{T} \frac{\mathrm{d} \phi_{I}^{\theta_{z_{i}}}}{\mathrm{~d} \xi_{i}} \phi_{J}^{v_{i}} \mathrm{~d} \xi_{i},} \\
{\left[\overline{\mathbf{M}}_{b}^{T}\right]_{I J}^{\theta_{y_{i}} \theta_{y_{i}}}=\int_{0}^{1} \lambda^{-2}\left(1+\chi_{2}^{T}\right) \phi_{I}^{\theta_{y_{i}}} \phi_{J}^{\theta_{y_{i}}} \mathrm{~d} \xi_{i},} \\
{\left[\overline{\mathbf{M}}_{b}^{T}\right]_{I J}^{\theta_{y_{i}} w_{i}}=\int_{0}^{1} \lambda^{-2} \chi_{3}^{T} \frac{\mathrm{d} \phi_{I}^{\theta_{y_{i}}}}{\mathrm{~d} \xi_{i}} \phi_{J}^{w_{i}} \mathrm{~d} \xi_{i},} \\
{\left[\overline{\mathbf{M}}_{b}^{T}\right]_{I J}^{v_{i} v_{i}}=\int_{0}^{1}\left(1+\chi_{1}^{T}\right) \phi_{I}^{v_{i}} \phi_{J}^{v_{i}} \mathrm{~d} \xi_{i},}
\end{gathered}
$$




$$
\begin{aligned}
& {\left[\overline{\mathbf{M}}_{b}^{T}\right]_{I J}^{w_{i} w_{i}}=\int_{0}^{1}\left(1+\chi_{1}^{T}\right) \phi_{I}^{w_{i}} \phi_{J}^{w_{i}} \mathrm{~d} \xi_{i}} \\
& {\left[\overline{\mathbf{K}}_{b}^{T}\right]_{I J}^{\theta_{z_{i}} \theta_{z_{i}}}=\int_{0}^{1}\left(\phi_{I}^{\theta_{z_{i}}} \phi_{J}^{\theta_{z_{i}}}+\left(\eta+\chi_{5}^{T}\right) \frac{\mathrm{d} \phi_{I}^{\theta_{z_{i}}}}{\mathrm{~d} \xi_{i}} \frac{\mathrm{d} \phi_{J}^{\theta_{z_{i}}}}{\mathrm{~d} \xi_{i}}\right) \mathrm{d} \xi_{i}} \\
& {\left[\overline{\mathbf{K}}_{b}^{T}\right]_{I J}^{\theta_{z_{i}} v_{i}}=-\int_{0}^{1}\left(\phi_{I}^{\theta_{z_{i}}} \phi_{J}^{v_{i}}+\chi_{4}^{T} \frac{\mathrm{d} \phi_{I}^{\theta_{z_{i}}}}{\mathrm{~d} \xi_{i}} \frac{\mathrm{d}^{2} \phi_{J}^{v_{i}}}{\mathrm{~d} \xi_{i}^{2}}\right) \mathrm{d} \xi_{i}} \\
& {\left[\overline{\mathbf{K}}_{b}^{T}\right]_{I J}^{v_{i} \theta_{z_{i}}}=-\int_{0}^{1} \phi_{I}^{v_{i}} \frac{\mathrm{d} \phi_{J}^{\theta_{z_{i}}}}{\mathrm{~d} \xi_{i}} \mathrm{~d} \xi_{i}} \\
& {\left[\overline{\mathbf{K}}_{b}^{T}\right]_{I J}^{v_{i} v_{i}}=\int_{0}^{1}\left(\left(1+\bar{T}^{T}+\chi_{6}^{T}\right) \frac{\mathrm{d} \phi_{I}^{v_{i}}}{\mathrm{~d} \xi_{i}} \frac{\mathrm{d} \phi_{J}^{v_{i}}}{\mathrm{~d} \xi_{i}}-\bar{f}_{1}^{T} \phi_{I}^{v_{i}} \phi_{J}^{v_{i}}\right) \mathrm{d} \xi_{i},} \\
& {\left[\overline{\mathbf{K}}_{b}^{T}\right]_{I J}^{v_{i} w_{i}}=-\int_{0}^{1} \bar{f}_{0}^{T} \phi_{I}^{w_{i}} \phi_{J}^{v_{i}} \mathrm{~d} \xi_{i}} \\
& {\left[\overline{\mathbf{K}}_{b}^{T}\right]_{I J}^{v_{i} v_{j}}=\int_{0}^{1} \bar{f}_{1}^{T} \phi_{I}^{v_{i}} \phi_{J}^{v_{j}} \mathrm{~d} \xi_{i} ; i \neq j} \\
& {\left[\overline{\mathbf{K}}_{b}^{T}\right]_{I J}^{\theta_{y_{i}} \theta_{y_{i}}}=\int_{0}^{1}\left(\phi_{I}^{\theta_{y_{i}}} \phi_{J}^{\theta_{y_{i}}}+\left(\eta+\chi_{5}^{T}\right) \frac{\mathrm{d} \phi_{I}^{\theta_{y_{i}}}}{\mathrm{~d} \xi_{i}} \frac{\mathrm{d} \phi_{J}^{\theta_{y_{i}}}}{\mathrm{~d} \xi_{i}}\right) \mathrm{d} \xi_{i},} \\
& {\left[\overline{\mathbf{K}}_{b}^{T}\right]_{I J}^{\theta_{y_{i}} w_{i}}=-\int_{0}^{1}\left(\phi_{I}^{\theta_{y_{i}}} \phi_{J}^{w_{i}}+\chi_{4}^{T} \frac{\mathrm{d} \phi_{I}^{\theta_{y_{i}}}}{\mathrm{~d} \xi_{i}} \frac{\mathrm{d}^{2} \phi_{J}^{w}}{\mathrm{~d} \xi_{i}^{2}}\right) \mathrm{d} \xi_{i},} \\
& {\left[\overline{\mathbf{K}}_{b}^{T}\right]_{I J}^{w_{i} \theta_{y_{i}}}=-\int_{0}^{1} \phi_{I}^{w_{i}} \frac{\mathrm{d} \phi_{J}^{\theta_{y_{i}}}}{\mathrm{~d} \xi_{i}} \mathrm{~d} \xi_{i}} \\
& {\left[\overline{\mathbf{K}}_{b}^{T}\right]_{I J}^{w_{i} w_{i}}=\int_{0}^{1}\left(\left(1+\bar{T}^{T}+\chi_{6}^{T}\right) \frac{\mathrm{d} \phi_{I}^{w_{i}}}{\mathrm{~d} \xi_{i}} \frac{\mathrm{d} \phi_{J}^{w_{i}}}{\mathrm{~d} \xi_{i}}+\bar{f}_{1}^{T} \phi_{I}^{w_{i}} \phi_{J}^{w_{i}}\right) \mathrm{d} \xi_{i}} \\
& {\left[\overline{\mathbf{K}}_{b}^{T}\right]_{I J}^{w_{i} v_{i}}=\int_{0}^{1} \bar{f}_{0}^{T} \phi_{I}^{v_{i}} \frac{\mathrm{d} \phi_{J}^{w_{i}}}{\mathrm{~d} \xi_{i}} \mathrm{~d} \xi_{i}} \\
& 31
\end{aligned}
$$




$$
\begin{gathered}
{\left[\overline{\mathbf{K}}_{b}^{T}\right]_{I J}^{w_{i} w_{j}}=-\int_{0}^{1} \bar{f}_{1}^{T} \phi_{I}^{w_{i}} \phi_{J}^{w_{j}} \mathrm{~d} \xi_{i} ; i \neq j} \\
\overline{\mathbf{\Theta}}_{z_{i}}^{T}=<\bar{\theta}_{z_{i 1}}^{T}, \bar{\theta}_{z_{i 2}}^{T}, \ldots, \bar{\theta}_{z_{i N P_{i}}}^{T}>^{\mathrm{T}} \\
\overline{\mathbf{v}}_{i}^{T}=<\bar{v}_{i 1}^{T}, \bar{v}_{i 2}^{T}, \ldots, \bar{v}_{i N P_{i}}^{T}>^{\mathrm{T}} \\
\overline{\boldsymbol{\Theta}}_{y_{i}}^{T}=<\bar{\theta}_{y_{i 1}}^{T}, \bar{\theta}_{y_{i 2}}^{T}, \ldots, \bar{\theta}_{y_{i N P_{i}}}^{T}>^{\mathrm{T}} \\
\overline{\mathbf{w}}_{i}^{T}=<\bar{w}_{i 1}^{T}, \bar{w}_{i 2}^{T}, \ldots, \bar{w}_{i N P_{i}}^{T}>^{\mathrm{T}}
\end{gathered}
$$

\subsubsection{Free transverse vibration on the basis of the proposed exact model}

In order to arrive at the discretized form of the exact model based on the TBT, both sides of Eqs. (44a)-(44h) in order are premultiplied by $\delta \bar{\theta}_{z_{1}}^{T}, \delta \bar{v}_{1}^{T}, \delta \bar{\theta}_{y_{1}}^{T}, \delta \bar{w}_{1}^{T}, \delta \bar{\theta}_{z_{2}}^{T}, \delta \bar{v}_{2}^{T}, \delta \bar{\theta}_{y_{2}}^{T}$, and $\delta \bar{w}_{2}^{T}$. After integrating from the resulting statements over the dimensionless longitudinal domains of the nanowires, taking the necessary integration by parts, and introducing Eq. (45) to the resulting relations, the set of ordinary differential equations of the problem is obtained as given in Eq. (46). All the mass submatrices and most of the stiffness submatrices are identical to those given in Eq. (47). Furthermore, the following stiffness submatrices are now modified based on the exact version of the applied magnetic forces:

$$
\begin{aligned}
& {\left[\overline{\mathbf{K}}_{b}^{T}\right]_{I J}^{v_{i} v_{i}}=\int_{0}^{1}\left(\left(1+\bar{T}^{T}+\chi_{6}^{T}\right) \frac{\mathrm{d} \phi_{I}^{v_{i}}}{\mathrm{~d} \xi_{i}} \frac{\mathrm{d} \phi_{J}^{v_{i}}}{\mathrm{~d} \xi_{i}}\right) \mathrm{d} \xi_{i}} \\
& +\frac{\bar{f}_{1}^{T}}{2 \bar{d}} \int_{0}^{1} \int_{0}^{1} \frac{\phi_{I}^{v_{i}}\left(\xi_{i}\right) \phi_{J}^{v_{i}}\left(\xi_{i}\right)}{\left(\left(\frac{\xi_{2}-\xi_{1}}{\bar{d}}\right)^{2}+1\right)^{\frac{3}{2}}} \mathrm{~d} \xi_{2} \mathrm{~d} \xi_{1}, \\
& {\left[\overline{\mathbf{K}}_{b}^{T}\right]_{I J}^{v_{i} v_{j}}=-\frac{\bar{f}_{1}^{T}}{2 \bar{d}} \int_{0}^{1} \int_{0}^{1} \frac{\phi_{I}^{v_{i}}\left(\left(\xi_{i}-\xi_{j}\right) \frac{\mathrm{d} \phi_{J}^{v_{j}}}{\mathrm{~d} \xi_{j}}+\phi_{J}^{v_{j}}\right)}{\left(\left(\frac{\xi_{2}-\xi_{1}}{\bar{d}}\right)^{2}+1\right)^{\frac{3}{2}}} \mathrm{~d} \xi_{i} \mathrm{~d} \xi_{j} ; i \neq j,}
\end{aligned}
$$




$$
\begin{gathered}
{\left[\overline{\mathbf{K}}_{b}^{T}\right]_{I J}^{w_{i} w_{i}}=\int_{0}^{1}\left(\left(1+\bar{T}^{T}+\chi_{6}^{T}\right) \frac{\mathrm{d} \phi_{I}^{w_{i}}}{\mathrm{~d} \xi} \frac{\mathrm{d} \phi_{J}^{w_{i}}}{\mathrm{~d} \xi}\right) \mathrm{d} \xi+} \\
+\frac{\bar{f}_{1}^{T}}{2 \bar{d}} \int_{0}^{1} \int_{0}^{1} \frac{\phi_{I}^{w_{i}}\left(\xi_{i}\right) \phi_{J}^{w_{i}}\left(\xi_{i}\right)}{\left(\left(\frac{\xi_{2}-\xi_{1}}{\bar{d}}\right)^{2}+1\right)^{\frac{3}{2}}} \mathrm{~d} \xi_{2} \mathrm{~d} \xi_{1}, \\
{\left[\overline{\mathbf{K}}_{b}^{T}\right]_{I J}^{w_{i} w_{j}}=-\frac{\bar{f}_{1}^{T}}{2 \bar{d}} \int_{0}^{1} \int_{0}^{1} \frac{\phi_{I}^{w_{i}}\left(\left(\xi_{i}-\xi_{j}\right) \frac{\mathrm{d} \phi_{J}^{w_{j}}}{\mathrm{~d} \xi_{j}}+\phi_{J}^{w_{j}}\right)}{\left(\left(\frac{\xi_{2}-\xi_{1}}{\bar{d}}\right)^{2}+1\right)^{\frac{3}{2}}} \mathrm{~d} \xi_{i} \mathrm{~d} \xi_{j} ; i \neq j .}
\end{gathered}
$$

\subsubsection{Enforcement of boundary conditions and frequency analysis}

For the CCNWs with simply supported ends, the following boundary conditions should be satisfied:

$$
\begin{aligned}
& \bar{w}_{i}^{T}(0, \tau)=\bar{w}_{i}^{T}(1, \tau)=0 ; \quad \bar{M}_{b_{y i}}^{T}(0, \tau)=\bar{M}_{b_{y i}}^{T}(1, \tau)=0, \\
& \bar{v}_{i}^{T}(0, \tau)=\bar{v}_{i}^{T}(1, \tau)=0 ; \quad \bar{M}_{b_{z i}}^{T}(0, \tau)=\bar{M}_{b_{z i}}^{T}(1, \tau)=0 ; i=1,2,
\end{aligned}
$$

where $\bar{M}_{b_{y i}}^{T}=\frac{M_{b_{y i}}^{T}}{k_{s} G_{b} A_{b} l_{b}}$ and $\bar{M}_{b_{z i}}^{T}=\frac{M_{b_{z i}}^{T}}{k_{s} G_{b} A_{b} l_{b}}$. In order to impose the essential boundary conditions in Eq. (49), the CCA is exploited. Thereafter, by assuming a harmonic form for the time-dependent vectors and solving the resulting eigenvalue equations, the natural frequencies of the magnetically affected nanosystem on the basis of the TBT could be easily determined.

\section{Development of surface energy-based formulations via HOBT}

\subsection{Constitutive relations of the bulk and the surface layer}

According to the HOBT of Reddy-Bickford (Bickford 1982; Reddy 1984), the components of the displacement field of both bulk and surface layer of the $i$ th CCNW are as:

$$
\begin{aligned}
& u_{x_{i}}^{H}\left(x_{i}, y_{i}, z_{i}, t\right)=-\left[\left(z_{i}-\alpha z_{i}^{3}\right) \psi_{y_{i}}^{H}\left(x_{i}, t\right)+\alpha z^{3} \frac{\partial w_{i}^{H}\left(x_{i}, t\right)}{\partial x_{i}}+\left(y_{i}-\alpha y_{i}^{3}\right) \psi_{z_{i}}^{H}\left(x_{i}, t\right)+\alpha y_{i}^{3} \frac{\partial v_{i}^{H}\left(x_{i}, t\right)}{\partial x_{i}}\right], \\
& u_{y_{i}}^{H}\left(x_{i}, y_{i}, z_{i}, t\right)=v_{i}^{H}\left(x_{i}, t\right), \quad u_{z_{i}}^{H}\left(x_{i}, y_{i}, z_{i}, t\right)=w_{i}^{H}\left(x_{i}, t\right),
\end{aligned}
$$


where $\psi_{y_{i}}^{H}$ and $\psi_{z_{i}}^{H}$ are the angles of rotation of the normal to the neutral axis about the $y_{i}$ and $z_{i}$ axes of the $i$ th CCNW, respectively, $v_{i}^{H}$ and $w_{i}^{H}$ represent the deflections of the $i$ th CCNW along the $y_{i}$ and $z_{i}$ axes, respectively, and $\alpha=4 /\left(3 D_{0}^{2}\right)$. The only linear strains within the bulk and the surface layer of the $i$ th CCNW are:

$$
\begin{aligned}
& \epsilon_{x x_{i}}^{H}=-\left[\left(z_{i}-\alpha z_{i}^{3}\right) \frac{\partial \psi_{y_{i}}^{H}}{\partial x_{i}}+\alpha z_{i}^{3} \frac{\partial^{2} w_{i}^{H}}{\partial x_{i}^{2}}+\left(y_{i}-\alpha y_{i}^{3}\right) \frac{\partial \psi_{z_{i}}^{H}}{\partial x_{i}}+\alpha y_{i}^{3} \frac{\partial^{2} v_{i}^{H}}{\partial x_{i}^{2}}\right], \\
& \gamma_{x y_{i}}^{H}=\left(1-3 \alpha y_{i}^{2}\right)\left(\frac{\partial v_{i}^{H}}{\partial x_{i}}-\psi_{z_{i}}^{H}\right), \quad \gamma_{x z_{i}}^{H}=\left(1-3 \alpha z_{i}^{2}\right)\left(\frac{\partial w_{i}^{H}}{\partial x_{i}}-\psi_{y_{i}}^{H}\right) .
\end{aligned}
$$

By exploiting the linear surface elasticity theory of Gurtin-Murdoch (Gurtin and Murdoch 1975, 1976, 1978), the stresses of the surface layer of the $i$ th CCNW are expressed by:

$$
\begin{aligned}
& \tau_{x x_{i}}^{H}=\tau_{0}+\left(\lambda_{0}+2 \mu_{0}\right) \frac{\partial u_{x_{i}}^{H}}{\partial x_{i}}=\tau_{0}-\left(\lambda_{0}+2 \mu_{0}\right)\left[\begin{array}{c}
\left(z_{i}-\alpha z_{i}^{3}\right) \frac{\partial \psi_{y_{i}}^{H}}{\partial x_{i}}+\alpha z_{i}^{3} \frac{\partial^{2} w_{i}^{H}}{\partial x_{i}^{2}}+ \\
\left(y_{i}-\alpha y_{i}^{3}\right) \frac{\partial \psi_{z_{i}}^{H}}{\partial x_{i}}+\alpha y_{i}^{3} \frac{\partial^{2} v_{i}^{H}}{\partial x_{i}^{2}}
\end{array}\right], \\
& \tau_{x y_{i}}^{H}=n_{y_{i}} \tau_{0} \frac{\partial v_{i}^{H}}{\partial x_{i}}, \quad \tau_{x z_{i}}^{H}=n_{z_{i}} \tau_{0} \frac{\partial w_{i}^{H}}{\partial x_{i}} .
\end{aligned}
$$

Let us assume that the bulk's normal stresses along the $y_{i}$ and $z_{i}$ axes would vary in a linear form across the thickness between those values of the surface. Hence, $\sigma_{y y_{i}}^{H}=$ $\frac{2 y}{D_{0}}\left(\tau_{0} \frac{\partial^{2} v_{i}^{H}}{\partial x_{i}^{2}}-\rho_{0} \frac{\partial^{2} v_{i}^{H}}{\partial t^{2}}\right)$ and $\sigma_{z z_{i}}^{H}=\frac{2 z}{D_{0}}\left(\tau_{0} \frac{\partial^{2} w_{i}^{H}}{\partial x_{i}^{2}}-\rho_{0} \frac{\partial^{2} w_{i}^{H}}{\partial t^{2}}\right)$. Additionally, the longitudinal normal stress within the bulk of the $i$ th CCNW reads: $\sigma_{x x_{i}}^{H}=E_{b} \epsilon_{x x_{i}}^{H}+\nu_{b}\left(\sigma_{y y_{i}}^{H}+\sigma_{z z_{i}}^{H}\right)$. By substituting the above-mentioned normal stresses into the recent relation, and using the given strains in Eq. (51), the longitudinal stress and the shear stresses of the bulk in terms of deformation field of the $i$ th CCNW would be:

$$
\begin{aligned}
\sigma_{x x_{i}}^{H}= & -E_{b}\left[\left(z_{i}-\alpha z_{i}^{3}\right) \frac{\partial \psi_{y_{i}}^{H}}{\partial x_{i}}+\alpha z_{i}^{3} \frac{\partial^{2} w_{i}^{H}}{\partial x_{i}^{2}}+\left(y_{i}-\alpha y_{i}^{3}\right) \frac{\partial \psi_{z_{i}}^{H}}{\partial x_{i}}+\alpha y_{i}^{3} \frac{\partial^{2} v_{i}^{H}}{\partial x_{i}^{2}}\right]+ \\
& \frac{2 \nu_{b}}{D_{0}}\left[y_{i}\left(\tau_{0} \frac{\partial^{2} v_{i}^{H}}{\partial x_{i}^{2}}-\rho_{0} \frac{\partial^{2} v_{i}^{H}}{\partial t^{2}}\right)+z_{i}\left(\tau_{0} \frac{\partial^{2} w_{i}^{H}}{\partial x_{i}^{2}}-\rho_{0} \frac{\partial^{2} w_{i}^{H}}{\partial t^{2}}\right)\right] \\
\sigma_{x y_{i}}^{H}= & \left(1-3 \alpha y_{i}^{2}\right) G_{b}\left(\frac{\partial v_{i}^{H}}{\partial x_{i}}-\psi_{z_{i}}^{H}\right), \quad \sigma_{x z_{i}}^{H}=\left(1-3 \alpha z_{i}^{2}\right) G_{b}\left(\frac{\partial w_{i}^{H}}{\partial x_{i}}-\psi_{y_{i}}^{H}\right) .
\end{aligned}
$$




\subsection{Equations of motion of magnetically affected DCCNWs using the HOBT}

In the context of theory of surface elasticity of Gurtin-Murdoch (Gurtin and Murdoch 1975, 1976, 1978), the kinetic energy, $T^{H}$, the elastic strain energy of the magnetically affected DCCNWs, $U^{H}$, and the work done by the resultant magnetic field on the nanosystem, $W^{H}$, are stated by:

$$
\begin{aligned}
& T^{H}=\frac{1}{2} \sum_{i=1}^{2} \int_{0}^{l_{b}}\left(\begin{array}{l}
\left(I_{0}+I_{0}^{*}\right)\left(\left(\frac{\partial^{2} v_{i}^{H}}{\partial t \partial x_{i}}\right)^{2}+\left(\frac{\partial^{2} w_{i}^{H}}{\partial t \partial x_{i}}\right)^{2}\right)+\left(I_{2}+I_{2}^{*}\right)\left(\left(\frac{\partial \psi_{y_{i}}^{H}}{\partial t}\right)^{2}+\left(\frac{\partial \psi_{z_{i}}^{H}}{\partial t}\right)^{2}\right)+ \\
\alpha^{2}\left(I_{6}+I_{6}^{*}\right)\left(\frac{\partial \psi_{y_{i}}^{H}}{\partial t}+\frac{\partial^{2} w_{i}^{H}}{\partial t \partial x_{i}}\right)^{2}-2 \alpha\left(I_{4}+I_{4}^{*}\right) \frac{\partial \psi_{y_{i}}^{H}}{\partial t}\left(\frac{\partial \psi_{y_{i}}^{H}}{\partial t}+\frac{\partial^{2} w_{i}^{H}}{\partial t \partial x_{i}}\right)+ \\
\alpha^{2}\left(I_{6}+I_{6}^{*}\right)\left(\frac{\partial \psi_{z_{i}}^{H}}{\partial t}+\frac{\partial^{2} v_{i}^{H}}{\partial t \partial x_{i}}\right)^{2}-2 \alpha\left(I_{4}+I_{4}^{*}\right) \frac{\partial \psi_{z_{i}}^{H}}{\partial t}\left(\frac{\partial \psi_{z_{i}}^{H}}{\partial t}+\frac{\partial^{2} v_{i}^{H}}{\partial t \partial x_{i}}\right)
\end{array}\right) \\
& U^{H=} \frac{1}{2} \sum_{i=1}^{2} \int_{0}^{l_{b}}\left(\begin{array}{l}
\frac{\partial \psi_{y_{i}}^{H}}{\partial x_{i}} M_{b_{y i}}^{H}+\left(\psi_{y_{i}}^{H}+\frac{\partial w_{i}^{H}}{\partial x_{i}}\right)\left(\alpha \frac{\partial P_{b_{z i}}^{H}}{\partial x_{i}}+Q_{z_{z i}}^{H}\right)+ \\
\frac{1}{\partial x_{i}} M_{b_{z i}}^{H}+\left(\psi_{z_{i}}^{H}+\frac{\partial v_{i}^{H}}{\partial x_{i}}\right)\left(\alpha \frac{\partial P_{b_{y i}}^{H}}{\partial x_{i}}+Q_{b_{y i}}^{H}\right)
\end{array}\right) \mathrm{d} x_{i}+ \\
& \frac{1}{2} \sum_{i=1}^{2} \int_{0}^{l_{b}} \int_{\mathcal{S}}\left(\tau_{x x_{i}}^{H} \epsilon_{x x_{i}}^{H}+\tau_{x z_{i}}^{H} \gamma_{x z_{i}}^{H}+\tau_{x y_{i}}^{H} \gamma_{x y_{i}}^{H}\right) \mathrm{d} \mathcal{S} \mathrm{d} x_{i}, \\
& W^{H}=\sum_{i=1}^{2} \int_{0}^{l_{b}}\left(v_{i}^{H} f_{m_{y i}}^{H}+w_{i}^{H} f_{m_{z i}}^{H}\right) \mathrm{d} x_{i},
\end{aligned}
$$

where

$$
\begin{aligned}
& M_{b_{z i}}^{H}=\int_{A_{b}} y_{i} \sigma_{x x_{i}}^{H} \mathrm{~d} A=-\left[\left(J_{2}-\alpha J_{4}\right) \frac{\partial \psi_{z_{i}}^{H}}{\partial x_{i}}+\alpha J_{4} \frac{\partial^{2} v_{i}^{H}}{\partial x_{i}^{2}}\right]+\frac{2 \nu_{b} I_{2}^{\prime}}{D_{0}}\left(\tau_{0} \frac{\partial^{2} v_{i}^{H}}{\partial x_{i}^{2}}-\rho_{0} \frac{\partial^{2} v_{i}^{H}}{\partial t^{2}}\right), \\
& M_{b_{y i}}^{H}=\int_{A_{b}} z_{i} \sigma_{x x_{i}}^{H} \mathrm{~d} A=-\left[\left(J_{2}-\alpha J_{4}\right) \frac{\partial \psi_{y_{i}}^{H}}{\partial x_{i}}+\alpha J_{4} \frac{\partial^{2} w_{i}^{H}}{\partial x_{i}^{2}}\right]+\frac{2 \nu_{b} I_{2}^{\prime}}{D_{0}}\left(\tau_{0} \frac{\partial^{2} w_{i}^{H}}{\partial x_{i}^{2}}-\rho_{0} \frac{\partial^{2} w_{i}^{H}}{\partial t^{2}}\right), \\
& P_{b_{z i}}^{H}=\int_{A_{b}} y_{i}^{3} \sigma_{x x_{i}}^{H} \mathrm{~d} A=-\left[\left(J_{4}-\alpha J_{6}\right) \frac{\partial \psi_{z_{i}}^{H}}{\partial x_{i}}+\alpha J_{6} \frac{\partial^{2} v_{i}^{H}}{\partial x_{i}^{2}}\right]+\frac{2 \nu_{b} I_{4}^{\prime}}{D_{0}}\left(\tau_{0} \frac{\partial^{2} v_{i}^{H}}{\partial x_{i}^{2}}-\rho_{0} \frac{\partial^{2} v_{i}^{H}}{\partial t^{2}}\right), \\
& P_{b_{y i}}^{H}=\int_{A_{b}} z_{i}^{3} \sigma_{x x_{i}}^{H} \mathrm{~d} A=-\left[\left(J_{4}-\alpha J_{6}\right) \frac{\partial \psi_{y_{i}}^{H}}{\partial x_{i}}+\alpha J_{6} \frac{\partial^{2} w_{i}^{H}}{\partial x_{i}^{2}}\right]+\frac{2 \nu_{b} I_{4}^{\prime}}{D_{0}}\left(\tau_{0} \frac{\partial^{2} w_{i}^{H}}{\partial x_{i}^{2}}-\rho_{0} \frac{\partial^{2} w_{i}^{H}}{\partial t^{2}}\right) \\
& Q_{b_{y i}}^{H}=\kappa\left(\frac{\partial v_{i}^{H}}{\partial x_{i}}-\psi_{z_{i}}^{H}\right), Q_{b_{z i}}^{H}=\kappa\left(\frac{\partial w_{i}^{H}}{\partial x_{i}}-\psi_{y_{i}}^{H}\right)
\end{aligned}
$$


and

$$
\begin{aligned}
& \kappa=\int_{A_{b}} G_{b}\left(1-3 \alpha z_{i}^{2}\right) \mathrm{d} A, I_{m}^{\prime}=\int_{A_{b}} z_{i}^{m} \mathrm{~d} A, I_{m}^{\prime *}=\int_{\mathcal{S}} z_{i}^{m} \mathrm{~d} \mathcal{S} ; m=2,4, \\
& J_{n}=\int_{A_{b}} E_{b} z_{i}^{n} \mathrm{~d} A, I_{n}=\int_{A_{b}} \rho_{b} z_{i}^{n} \mathrm{~d} A, I_{n}^{*}=\int_{\mathcal{S}} \rho_{0} z_{i}^{n} \mathrm{~d} \mathcal{S} ; n=0,2,4,6 .
\end{aligned}
$$

Using Hamilton's principle, the equations of motion of the magnetically affected DCCNWs according to the HOBT are obtained as follows:

$$
\begin{aligned}
& \frac{\partial M_{b_{z i}}^{H}}{\partial x_{i}}-\alpha \frac{\partial P_{b_{y i}}^{H}}{\partial x_{i}}-Q_{b_{y i}}^{H}+\int_{\mathcal{S}} y \frac{\partial \tau_{x x_{i}}^{H}}{\partial x_{i}} \mathrm{~d} \mathcal{S}=\left(\left(I_{2}+I_{2}^{*}\right)-2 \alpha\left(I_{4}+I_{4}^{*}\right)+\right. \\
& \left.\alpha^{2}\left(I_{6}+I_{6}^{*}\right)\right) \frac{\partial^{2} \psi_{z_{i}}^{H}}{\partial t^{2}}+\left(\alpha^{2}\left(I_{6}+I_{6}^{*}\right)-\alpha\left(I_{4}+I_{4}^{*}\right)\right) \frac{\partial^{3} v_{i}^{H}}{\partial t^{2} \partial x_{i}}, \\
& -\frac{\partial Q_{b_{y i}}^{H}}{\partial x_{i}}-\alpha \frac{\partial^{2} P_{b_{y i}}^{H}}{\partial x_{i}^{2}}-T \frac{\partial^{2} v_{i}^{H}}{\partial x_{i}^{2}}-\int_{\mathcal{S}} \frac{\partial \tau_{x y_{i}}^{H}}{\partial x_{i}} n_{y_{i}} \mathrm{~d} \mathcal{S}= \\
& f_{m_{y i}}^{H}-\left(I_{0}+I_{0}^{*}\right) \frac{\partial^{2} v_{i}^{H}}{\partial t^{2}}-\left(\alpha I_{4}-\alpha^{2} I_{6}\right) \frac{\partial^{3} \psi_{z_{i}}^{H}}{\partial t^{2} \partial x_{i}} \\
& \frac{\partial M_{b_{y i}}^{H}}{\partial x_{i}}-\alpha \frac{\partial P_{b_{z i}}^{H}}{\partial x}-Q_{b_{y i}}^{H}+\int_{\mathcal{S}} z_{i} \frac{\partial \tau_{x x_{i}}^{H}}{\partial x_{i}} \mathrm{~d} \mathcal{S}=\left(\left(I_{2}+I_{2}^{*}\right)-2 \alpha\left(I_{4}+I_{4}^{*}\right)+\right. \\
& \left.\alpha^{2}\left(I_{6}+I_{6}^{*}\right)\right) \frac{\partial^{2} \psi_{y_{i}}^{H}}{\partial t^{2}}+\left(\alpha^{2}\left(I_{6}+I_{6}^{*}\right)-\alpha\left(I_{4}+I_{4}^{*}\right)\right) \frac{\partial^{3} w_{i}^{H}}{\partial t^{2} \partial x_{i}}, \\
& -\frac{\partial Q_{b_{z i}}^{H}}{\partial x_{i}}-\alpha \frac{\partial^{2} P_{b_{z i}}^{H}}{\partial x_{i}^{2}}-T \frac{\partial^{2} w_{i}^{H}}{\partial x_{i}^{2}}-\int_{\mathcal{S}} \frac{\partial \tau_{x z_{i}}^{H}}{\partial x_{i}} n_{z_{i}} \mathrm{~d} \mathcal{S}= \\
& f_{m_{z i}}^{H}-\left(I_{0}+I_{0}^{*}\right) \frac{\partial^{2} w_{i}^{H}}{\partial t^{2}}-\left(\alpha I_{4}-\alpha^{2} I_{6}\right) \frac{\partial^{3} \psi_{y_{i}}^{H}}{\partial t^{2} \partial x_{i}} ; i=1,2 .
\end{aligned}
$$

\subsubsection{An approximate version of the governing equations based on the HOBT}

By substituting Eqs. (10a), (10b), (52), and (55) into Eqs. (57a)-(57d), the approximate version of the governing equations describe transverse vibrations of DCCNWs acted upon by a longitudinal magnetic field in terms of deformation fields of the HOBT are derived as:

$$
\begin{aligned}
& \left(I_{2}+I_{2}^{*}-2 \alpha\left(I_{4}+I_{4}^{*}\right)+\alpha^{2}\left(I_{6}+I_{6}^{*}\right)\right) \frac{\partial^{2} \psi_{z_{1}}^{H}}{\partial t^{2}}+\left(\alpha^{2}\left(I_{6}+I_{6}^{*}\right)-\alpha\left(I_{4}+I_{4}^{*}\right)\right) \frac{\partial^{3} v_{1}^{H}}{\partial t^{2} \partial x_{1}}- \\
& \left(\alpha J_{4}-\alpha^{2} J_{6}+\left(\lambda_{0}+2 \mu_{0}\right) \alpha I_{4}^{\prime *}-\frac{2 \nu_{b} \tau_{0}}{D_{0}}\left(I_{2}^{\prime}-\alpha I_{4}^{\prime}\right)\right) \frac{\partial^{3} v_{1}^{H}}{\partial x_{1}^{3}}-\left(J_{2}-2 \alpha J_{4}+\alpha^{2} J_{6}+\right. \\
& \left.\left(\lambda_{0}+2 \mu_{0}\right)\left(I_{2}^{\prime *}-\alpha I_{4}^{\prime *}\right)\right) \frac{\partial^{2} \psi_{z_{1}}^{H}}{\partial x_{1}^{2}}-\kappa\left(\frac{\partial v_{1}^{H}}{\partial x_{1}}-\psi_{z_{1}}^{H}\right)=0
\end{aligned}
$$




$$
\begin{aligned}
& \left(I_{0}+I_{0}^{*}+\frac{2 \alpha \nu_{b} I_{4}^{\prime} \rho_{0}}{D_{0}}\right) \frac{\partial^{2} v_{1}^{H}}{\partial t^{2}}+\left(\alpha I_{4}-\alpha^{2} I_{6}\right) \frac{\partial^{3} \psi_{z_{1}}^{H}}{\partial t^{2} \partial x_{1}}-\kappa\left(\frac{\partial^{2} v_{1}^{H}}{\partial x_{1}^{2}}-\frac{\partial \psi_{z_{1}}^{H}}{\partial x_{1}}\right)- \\
& \left(T+\tau_{0} S_{0}^{*}+\alpha^{2} J_{6}\right) \frac{\partial^{2} v_{1}^{H}}{\partial x_{1}^{2}}+\left(\alpha J_{4}-\alpha^{2} J_{6}\right) \frac{\partial^{3} \psi_{z_{1}}^{H}}{\partial x_{1}^{3}}+\left(\alpha^{2} J_{6}-\frac{2 \alpha \nu_{b} I_{4}^{\prime} \tau_{0}}{D_{0}}\right) \frac{\partial^{4} v_{1}^{H}}{\partial x_{1}^{4}} \\
& -B_{0} I_{0} \frac{\partial w_{1}^{H}}{\partial x_{1}}+\frac{\mu_{0} I_{0}^{2}}{2 \pi d^{2}}\left(v_{2}^{H}-v_{1}^{H}\right)=0, \\
& \left(I_{2}+I_{2}^{*}-2 \alpha\left(I_{4}+I_{4}^{*}\right)+\alpha^{2}\left(I_{6}+I_{6}^{*}\right)\right) \frac{\partial^{2} \psi_{y_{1}}^{H}}{\partial t^{2}}+\left(\alpha^{2}\left(I_{6}+I_{6}^{*}\right)-\alpha\left(I_{4}+I_{4}^{*}\right)\right) \frac{\partial^{3} w_{1}^{H}}{\partial t^{2} \partial x_{1}}- \\
& \left(\alpha J_{4}-\alpha^{2} J_{6}+\left(\lambda_{0}+2 \mu_{0}\right) \alpha I_{4}^{\prime *}-\frac{2 \nu_{b} \tau_{0}}{D_{0}}\left(I_{2}^{\prime}-\alpha I_{4}^{\prime}\right)\right) \frac{\partial^{3} w_{1}^{H}}{\partial x_{1}^{3}}-\left(J_{2}-2 \alpha J_{4}+\alpha^{2} J_{6}+\right. \\
& \left.\left(\lambda_{0}+2 \mu_{0}\right)\left(I_{2}^{* *}-\alpha I_{4}^{\prime *}\right)\right) \frac{\partial^{2} \psi_{y_{1}}^{H}}{\partial x_{1}^{2}}-\kappa\left(\frac{\partial w_{1}^{H}}{\partial x_{1}}-\psi_{y_{1}}^{H}\right)=0, \\
& \left(I_{0}+I_{0}^{*}+\frac{2 \alpha \nu_{b} I_{4}^{\prime} \rho_{0}}{D_{0}}\right) \frac{\partial^{2} w_{1}^{H}}{\partial t^{2}}+\left(\alpha I_{4}-\alpha^{2} I_{6}\right) \frac{\partial^{3} \psi_{y_{1}}^{H}}{\partial t^{2} \partial x_{1}}-\kappa\left(\frac{\partial^{2} w_{1}^{H}}{\partial x_{1}^{2}}-\frac{\partial \psi_{y_{1}}^{H}}{\partial x_{1}}\right)- \\
& \left(T+\tau_{0} S_{0}^{*}+\alpha^{2} J_{6}\right) \frac{\partial^{2} w_{1}^{H}}{\partial x_{1}^{2}}+\left(\alpha J_{4}-\alpha^{2} J_{6}\right) \frac{\partial^{3} \psi_{y_{1}}^{H}}{\partial x_{1}^{3}}+\left(\alpha^{2} J_{6}-\frac{2 \alpha \nu_{b} I_{4}^{\prime} \tau_{0}}{D_{0}}\right) \frac{\partial^{4} w_{1}^{H}}{\partial x_{1}^{4}}+ \\
& B_{0} I_{0} \frac{\partial v_{1}^{H}}{\partial x_{1}}-\frac{\mu_{0} I_{0}^{2}}{2 \pi d^{2}}\left(w_{2}^{H}-w_{1}^{H}\right)=0 \\
& \left(I_{2}+I_{2}^{*}-2 \alpha\left(I_{4}+I_{4}^{*}\right)+\alpha^{2}\left(I_{6}+I_{6}^{*}\right)\right) \frac{\partial^{2} \psi_{z_{2}}^{H}}{\partial t^{2}}+\left(\alpha^{2}\left(I_{6}+I_{6}^{*}\right)-\alpha\left(I_{4}+I_{4}^{*}\right)\right) \frac{\partial^{3} v_{2}^{H}}{\partial t^{2} \partial x_{2}}- \\
& \left(\alpha J_{4}-\alpha^{2} J_{6}+\left(\lambda_{0}+2 \mu_{0}\right) \alpha I_{4}^{\prime *}-\frac{2 \nu_{b} \tau_{0}}{D_{0}}\left(I_{2}^{\prime}-\alpha I_{4}^{\prime}\right)\right) \frac{\partial^{3} v_{2}^{H}}{\partial x_{2}^{3}}-\left(J_{2}-2 \alpha J_{4}+\alpha^{2} J_{6}+\right. \\
& \left.\left(\lambda_{0}+2 \mu_{0}\right)\left(I_{2}^{\prime *}-\alpha I_{4}^{\prime *}\right)\right) \frac{\partial^{2} \psi_{z_{2}}^{H}}{\partial x_{2}^{2}}-\kappa\left(\frac{\partial v_{2}^{H}}{\partial x_{2}}-\psi_{z_{2}}^{H}\right)=0 \\
& \left(I_{0}+I_{0}^{*}+\frac{2 \alpha \nu_{b} I_{4}^{\prime} \rho_{0}}{D_{0}}\right) \frac{\partial^{2} v_{2}^{H}}{\partial t^{2}}+\left(\alpha I_{4}-\alpha^{2} I_{6}\right) \frac{\partial^{3} \psi_{z_{2}}^{H}}{\partial t^{2} \partial x_{2}}-\kappa\left(\frac{\partial^{2} v_{2}^{H}}{\partial x_{2}^{2}}-\frac{\partial \psi_{z_{2}}^{H}}{\partial x_{2}}\right)- \\
& \left(T+\tau_{0} S_{0}^{*}+\alpha^{2} J_{6}\right) \frac{\partial^{2} v_{2}^{H}}{\partial x_{2}^{2}}+\left(\alpha J_{4}-\alpha^{2} J_{6}\right) \frac{\partial^{3} \psi_{z_{2}}^{H}}{\partial x_{2}^{3}}+\left(\alpha^{2} J_{6}-\frac{2 \alpha \nu_{b} I_{4}^{\prime} \tau_{0}}{D_{0}}\right) \frac{\partial^{4} v_{2}^{H}}{\partial x_{2}^{4}} \\
& -B_{0} I_{0} \frac{\partial w_{2}^{H}}{\partial x_{2}}-\frac{\mu_{0} I_{0}^{2}}{2 \pi d^{2}}\left(v_{2}^{H}-v_{1}^{H}\right)=0, \\
& \left(I_{2}+I_{2}^{*}-2 \alpha\left(I_{4}+I_{4}^{*}\right)+\alpha^{2}\left(I_{6}+I_{6}^{*}\right)\right) \frac{\partial^{2} \psi_{y_{2}}^{H}}{\partial t^{2}}+\left(\alpha^{2}\left(I_{6}+I_{6}^{*}\right)-\alpha\left(I_{4}+I_{4}^{*}\right)\right) \frac{\partial^{3} w_{2}^{H}}{\partial t^{2} \partial x_{2}}- \\
& \left(\alpha J_{4}-\alpha^{2} J_{6}+\left(\lambda_{0}+2 \mu_{0}\right) \alpha I_{4}^{\prime *}-\frac{2 \nu_{b} \tau_{0}}{D_{0}}\left(I_{2}^{\prime}-\alpha I_{4}^{\prime}\right)\right) \frac{\partial^{3} w_{2}^{H}}{\partial x_{2}^{3}}-\left(J_{2}-2 \alpha J_{4}+\alpha^{2} J_{6}+\right. \\
& \left.\left(\lambda_{0}+2 \mu_{0}\right)\left(I_{2}^{\prime *}-\alpha I_{4}^{* *}\right)\right) \frac{\partial^{2} \psi_{y_{2}}^{H}}{\partial x_{2}^{2}}-\kappa\left(\frac{\partial w_{2}^{H}}{\partial x_{2}}-\psi_{y_{2}}^{H}\right)=0
\end{aligned}
$$




$$
\begin{aligned}
& \left(I_{0}+I_{0}^{*}+\frac{2 \alpha \nu_{b} I_{4}^{\prime} \rho_{0}}{D_{0}}\right) \frac{\partial^{2} w_{2}^{H}}{\partial t^{2}}+\left(\alpha I_{4}-\alpha^{2} I_{6}\right) \frac{\partial^{3} \psi_{y_{2}}^{H}}{\partial t^{2} \partial x_{2}}-\kappa\left(\frac{\partial^{2} w_{2}^{H}}{\partial x_{2}^{2}}-\frac{\partial \psi_{y_{2}}^{H}}{\partial x_{2}}\right)- \\
& \left(T+\tau_{0} S_{0}^{*}+\alpha^{2} J_{6}\right) \frac{\partial^{2} w_{2}^{H}}{\partial x_{2}^{2}}+\left(\alpha J_{4}-\alpha^{2} J_{6}\right) \frac{\partial^{3} \psi_{y_{2}}^{H}}{\partial x_{2}^{3}}+\left(\alpha^{2} J_{6}-\frac{2 \alpha \nu_{b} I_{4}^{\prime} \tau_{0}}{D_{0}}\right) \frac{\partial^{4} w_{2}^{H}}{\partial x_{2}^{4}}+ \\
& B_{0} I_{0} \frac{\partial v_{2}^{H}}{\partial x_{2}}+\frac{\mu_{0} I_{0}^{2}}{2 \pi d^{2}}\left(w_{2}^{H}-w_{1}^{H}\right)=0 .
\end{aligned}
$$

To scrutinize the problem in a more systematic manner, the following dimensionless quantities are taken into account:

$$
\begin{aligned}
& \bar{v}_{i}^{H}=\frac{v_{i}^{H}}{l_{b}}, \bar{w}_{i}^{H}=\frac{w_{i}^{H}}{l_{b}}, \bar{\psi}_{y_{i}}^{H}=\psi_{y_{i}}^{H}, \bar{\psi}_{z_{i}}^{H}=\psi_{z_{i}}^{H}, \tau=\frac{\alpha t}{l_{b}^{2}} \sqrt{\frac{J_{6}}{I_{0}}}, \gamma_{1}^{2}=\frac{\alpha I_{4}-\alpha^{2} I_{6}}{I_{0} l_{b}^{2}}, \\
& \gamma_{2}^{2}=\frac{\alpha^{2} I_{6}}{I_{0} l_{b}^{2}}, \gamma_{3}^{2}=\frac{\kappa l_{b}^{2}}{\alpha^{2} J_{6}}, \gamma_{4}^{2}=\frac{\alpha J_{4}-\alpha^{2} J_{6}}{\alpha^{2} J_{6}}, \gamma_{6}^{2}=\frac{\alpha I_{4}-\alpha^{2} I_{6}}{I_{2}-2 \alpha I_{4}+\alpha^{2} I_{6}}, \\
& \gamma_{7}^{2}=\frac{\kappa I_{0} l_{b}^{4}}{\left(I_{2}-2 \alpha I_{4}+\alpha^{2} I_{6}\right) \alpha^{2} J_{6}}, \gamma_{8}^{2}=\frac{\left(J_{2}-2 \alpha J_{4}+\alpha^{2} J_{6}\right) I_{0} l_{b}^{2}}{\left(I_{2}-2 \alpha I_{4}+\alpha^{2} I_{6}\right) \alpha^{2} J_{6}}, \gamma_{9}^{2}=\frac{\left(\alpha J_{4}-\alpha^{2} J_{6}\right) I_{0} l_{b}^{2}}{\left(I_{2}-2 \alpha I_{4}+\alpha^{2} I_{6}\right) \alpha^{2} J_{6}}, \\
& \bar{T}^{H}=\frac{T l_{b}^{3}}{\alpha^{2} J_{6}}, \bar{f}_{0}^{H}=\frac{B_{0} I_{0} l_{b}^{3}}{\alpha^{2} J_{6}}, \bar{f}_{1}^{H}=\frac{\mu_{0} I_{0}^{2} l_{b}^{4}}{2 \pi d^{2} \alpha^{2} J_{6}}, \chi_{1}^{H}=\frac{I_{2}^{*}-2 \alpha I_{4}^{*}+\alpha^{2} I_{6}^{*}}{I_{2}-2 \alpha I_{4}+\alpha^{2} I_{6}}, \\
& \chi_{2}^{H}=\frac{\alpha I_{4}^{*}-\alpha^{2} I_{6}^{*}-\frac{2 \nu_{b} \rho_{0}}{D_{0}}\left(I_{2}^{\prime}-\alpha I_{4}^{\prime}\right)}{\alpha I_{4}-\alpha^{2} I_{6}}, \chi_{3}^{H}=\frac{\left(\lambda_{0}+2 \mu_{0}\right)-\frac{2 \nu_{b} \tau_{0}}{D_{0}}\left(I_{2}^{\prime}-\alpha I_{4}^{\prime}\right)}{\alpha J_{4}-\alpha^{2} J_{6}}, \\
& \chi_{4}^{H}=\frac{\left(\lambda_{0}+2 \mu_{0}\right)\left(I_{2}^{*}-\alpha I_{4}^{*}\right)}{J_{2}-2 \alpha J_{4}+\alpha^{2} J_{6}}, \chi_{5}^{H}=\frac{\rho_{0} S_{0}^{*}+\frac{2 \alpha \nu_{b} I_{4}^{\prime} \rho_{0}}{D_{0}}}{I_{0}}, \chi_{6}^{H}=\frac{\tau_{0} S_{0}^{*} l_{b}^{3}}{\alpha^{2} J_{6}}, \chi_{7}^{H}=\frac{2 \nu_{b} I_{4}^{\prime} \tau_{0} l_{b}^{3}}{\alpha J_{6} D_{0}} .
\end{aligned}
$$

As a result, introduction of Eq. (59) into Eqs. (58a)-(58h) leads to the dimensionless equations of motion of DCCNWs immersed in a longitudinal magnetic field on the basis of the HOBT:

$$
\begin{gathered}
\left(1+\chi_{1}^{H}\right) \frac{\partial^{2} \bar{\psi}_{z_{1}}^{H}}{\partial \tau^{2}}+\gamma_{6}^{2}\left(1+\chi_{2}^{H}\right) \frac{\partial^{3} \bar{v}_{1}^{H}}{\partial \tau^{2} \partial \xi_{1}}-\gamma_{9}^{2}\left(1+\chi_{3}^{H}\right) \frac{\partial^{3} \bar{v}_{1}^{H}}{\partial \xi_{1}^{3}} \\
-\gamma_{8}^{2}\left(1+\chi_{4}^{H}\right) \frac{\partial^{2} \bar{\psi}_{z_{1}}^{H}}{\partial \xi_{1}^{2}}-\gamma_{7}^{2}\left(\frac{\partial \bar{v}_{1}^{H}}{\partial \xi_{1}}-\bar{\psi}_{z_{1}}^{H}\right)=0 \\
\left(1+\chi_{5}^{H}\right) \frac{\partial^{2} \bar{v}_{1}^{H}}{\partial \tau^{2}}-\gamma_{2}^{2} \frac{\partial^{4} \bar{v}_{1}^{H}}{\partial \tau^{2} \partial \xi_{1}^{2}}+\gamma_{1}^{2} \frac{\partial^{3} \bar{\psi}_{z_{1}}^{H}}{\partial \tau^{2} \partial \xi_{1}}-\gamma_{3}^{2}\left(\frac{\partial^{2} \bar{v}_{1}^{H}}{\partial \xi_{1}^{2}}-\frac{\partial \bar{\psi}_{z_{1}}^{H}}{\partial \xi_{1}}\right) \\
-\left(\bar{T}^{H}+\chi_{6}^{H}\right) \frac{\partial^{2} \bar{v}_{1}^{H}}{\partial \xi_{1}^{2}}+\gamma_{4}^{2} \frac{\partial^{3} \bar{\psi}_{z_{1}}^{H}}{\partial \xi_{1}^{3}}+\left(1-\chi_{7}^{H}\right) \frac{\partial^{4} \bar{v}_{1}^{H}}{\partial \xi_{1}^{4}}-\bar{f}_{0}^{H} \frac{\partial \bar{w}_{1}^{H}}{\partial \xi_{1}}+\bar{f}_{1}^{H}\left(\bar{v}_{2}^{H}-\bar{v}_{1}^{H}\right)=0,
\end{gathered}
$$




$$
\begin{aligned}
& \left(1+\chi_{1}^{H}\right) \frac{\partial^{2} \bar{\psi}_{y_{1}}^{H}}{\partial \tau^{2}}+\gamma_{6}^{2}\left(1+\chi_{2}^{H}\right) \frac{\partial^{3} \bar{w}_{1}^{H}}{\partial \tau^{2} \partial \xi_{1}}-\gamma_{9}^{2}\left(1+\chi_{3}^{H}\right) \frac{\partial^{3} \bar{w}_{1}^{H}}{\partial \xi_{1}^{3}} \\
& -\gamma_{8}^{2}\left(1+\chi_{4}^{H}\right) \frac{\partial^{2} \bar{\psi}_{y_{1}}^{H}}{\partial \xi_{1}^{2}}-\gamma_{7}^{2}\left(\frac{\partial \bar{w}_{1}^{H}}{\partial \xi_{1}}-\bar{\psi}_{y_{1}}^{H}\right)=0 \\
& \left(1+\chi_{5}^{H}\right) \frac{\partial^{2} \bar{w}_{1}^{H}}{\partial \tau^{2}}-\gamma_{2}^{2} \frac{\partial^{4} \bar{w}_{1}^{H}}{\partial \tau^{2} \partial \xi_{1}^{2}}+\gamma_{1}^{2} \frac{\partial^{3} \bar{\psi}_{y_{1}}^{H}}{\partial \tau^{2} \partial \xi_{1}}-\gamma_{3}^{2}\left(\frac{\partial^{2} \bar{w}_{1}^{H}}{\partial \xi_{1}^{2}}-\frac{\partial \bar{\psi}_{y_{1}}^{H}}{\partial \xi_{1}}\right) \\
& -\left(\bar{T}^{H}+\chi_{6}^{H}\right) \frac{\partial^{2} \bar{w}_{1}^{H}}{\partial \xi_{1}^{2}}+\gamma_{4}^{2} \frac{\partial^{3} \bar{\psi}_{y_{1}}^{H}}{\partial \xi_{1}^{3}}+\left(1-\chi_{7}^{H}\right) \frac{\partial^{4} \bar{w}_{1}^{H}}{\partial \xi_{1}^{4}}+\bar{f}_{0}^{H} \frac{\partial \bar{v}_{1}^{H}}{\partial \xi_{1}}-\bar{f}_{1}^{H}\left(\bar{w}_{2}^{H}-\bar{w}_{1}^{H}\right)=0, \\
& \left(1+\chi_{1}^{H}\right) \frac{\partial^{2} \bar{\psi}_{z_{2}}^{H}}{\partial \tau^{2}}+\gamma_{6}^{2}\left(1+\chi_{2}^{H}\right) \frac{\partial^{3} \bar{v}_{2}^{H}}{\partial \tau^{2} \partial \xi_{2}}-\gamma_{9}^{2}\left(1+\chi_{3}^{H}\right) \frac{\partial^{3} \bar{v}_{2}^{H}}{\partial \xi_{2}^{3}} \\
& -\gamma_{8}^{2}\left(1+\chi_{4}^{H}\right) \frac{\partial^{2} \bar{\psi}_{z_{2}}^{H}}{\partial \xi_{2}^{2}}-\gamma_{7}^{2}\left(\frac{\partial \bar{v}_{2}^{H}}{\partial \xi_{2}}-\bar{\psi}_{z_{2}}^{H}\right)=0 \\
& \left(1+\chi_{5}^{H}\right) \frac{\partial^{2} \bar{v}_{2}^{H}}{\partial \tau^{2}}-\gamma_{2}^{2} \frac{\partial^{4} \bar{v}_{2}^{H}}{\partial \tau^{2} \partial \xi_{2}^{2}}+\gamma_{1}^{2} \frac{\partial^{3} \bar{\psi}_{z_{2}}^{H}}{\partial \tau^{2} \partial \xi_{2}}-\gamma_{3}^{2}\left(\frac{\partial^{2} \bar{v}_{2}^{H}}{\partial \xi_{2}^{2}}-\frac{\partial \bar{\psi}_{z_{2}}^{H}}{\partial \xi_{2}}\right) \\
& -\left(\bar{T}^{H}+\chi_{6}^{H}\right) \frac{\partial^{2} \bar{v}_{2}^{H}}{\partial \xi_{2}^{2}}+\gamma_{4}^{2} \frac{\partial^{3} \bar{\psi}_{z_{2}}^{H}}{\partial \xi_{2}^{3}}+\left(1-\chi_{7}^{H}\right) \frac{\partial^{4} \bar{v}_{2}^{H}}{\partial \xi_{2}^{4}}-\bar{f}_{0}^{H} \frac{\partial \bar{w}_{2}^{H}}{\partial \xi_{2}}-\bar{f}_{1}^{H}\left(\bar{v}_{2}^{H}-\bar{v}_{1}^{H}\right)=0 \\
& \left(1+\chi_{1}^{H}\right) \frac{\partial^{2} \bar{\psi}_{y_{2}}^{H}}{\partial \tau^{2}}+\gamma_{6}^{2}\left(1+\chi_{2}^{H}\right) \frac{\partial^{3} \bar{w}_{2}^{H}}{\partial \tau^{2} \partial \xi_{2}}-\gamma_{9}^{2}\left(1+\chi_{3}^{H}\right) \frac{\partial^{3} \bar{w}_{2}^{H}}{\partial \xi_{2}^{3}} \\
& -\gamma_{8}^{2}\left(1+\chi_{4}^{H}\right) \frac{\partial^{2} \bar{\psi}_{y_{2}}^{H}}{\partial \xi_{2}^{2}}-\gamma_{7}^{2}\left(\frac{\partial \bar{w}_{2}^{H}}{\partial \xi_{2}}-\bar{\psi}_{y_{2}}^{H}\right)=0 \\
& \left(1+\chi_{5}^{H}\right) \frac{\partial^{2} \bar{w}_{2}^{H}}{\partial \tau^{2}}-\gamma_{2}^{2} \frac{\partial^{4} \bar{w}_{2}^{H}}{\partial \tau^{2} \partial \xi_{2}^{2}}+\gamma_{1}^{2} \frac{\partial^{3} \bar{\psi}_{y_{2}}^{H}}{\partial \tau^{2} \partial \xi_{2}}-\gamma_{3}^{2}\left(\frac{\partial^{2} \bar{w}_{2}^{H}}{\partial \xi_{2}^{2}}-\frac{\partial \bar{\psi}_{y_{2}}^{H}}{\partial \xi_{2}}\right) \\
& -\left(\bar{T}^{H}+\chi_{6}^{H}\right) \frac{\partial^{2} \bar{w}_{2}^{H}}{\partial \xi_{2}^{2}}+\gamma_{4}^{2} \frac{\partial^{3} \bar{\psi}_{y_{2}}^{H}}{\partial \xi_{2}^{3}}+\left(1-\chi_{7}^{H}\right) \frac{\partial^{4} \bar{w}_{2}^{H}}{\partial \xi_{2}^{4}}+\bar{f}_{0}^{H} \frac{\partial \bar{v}_{2}^{H}}{\partial \xi_{2}}+\bar{f}_{1}^{H}\left(\bar{w}_{2}^{H}-\bar{w}_{1}^{H}\right)=0 .
\end{aligned}
$$

In the absence of the surface parameters (i.e., $\chi_{i}^{H}=0 ; i=1,2, \ldots, 7$ ), the dimensionless equations of motion of doubly parallel macro-wires subjected to a longitudinal magnetic field based on the HOBT are retrieved. 


\subsubsection{An exact version of the governing equations based on the HOBT}

To obtain the exact governing equations, the exact version of the interwire magnetic forces is employed. For this purpose, Eqs. (14a) and (14b) plus to (52), and (55) are introduced to Eqs. (57a)-(57d). Hence, the exact equations of motion display transverse vibrations of magnetically affected DCCNWs on the basis of the HOBT are derived as follows:

$$
\begin{aligned}
& \left(I_{2}+I_{2}^{*}-2 \alpha\left(I_{4}+I_{4}^{*}\right)+\alpha^{2}\left(I_{6}+I_{6}^{*}\right)\right) \frac{\partial^{2} \psi_{z_{1}}^{H}}{\partial t^{2}}+\left(\alpha^{2}\left(I_{6}+I_{6}^{*}\right)-\alpha\left(I_{4}+I_{4}^{*}\right)\right) \frac{\partial^{3} v_{1}^{H}}{\partial t^{2} \partial x_{1}}- \\
& \left(\alpha J_{4}-\alpha^{2} J_{6}+\left(\lambda_{0}+2 \mu_{0}\right) \alpha I_{4}^{\prime *}-\frac{2 \nu_{b} \tau_{0}}{D_{0}}\left(I_{2}^{\prime}-\alpha I_{4}^{\prime}\right)\right) \frac{\partial^{3} v_{1}^{H}}{\partial x_{1}^{3}}-\left(J_{2}-2 \alpha J_{4}+\alpha^{2} J_{6}+\right. \\
& \left.\left(\lambda_{0}+2 \mu_{0}\right)\left(I_{2}^{*}-\alpha I_{4}^{*}\right)\right) \frac{\partial^{2} \psi_{z_{1}}^{H}}{\partial x_{1}^{2}}-\kappa\left(\frac{\partial v_{1}^{H}}{\partial x_{1}}-\psi_{z_{1}}^{H}\right)=0 \\
& \left(I_{0}+I_{0}^{*}+\frac{2 \alpha \nu_{b} I_{4}^{\prime} \rho_{0}}{D_{0}}\right) \frac{\partial^{2} v_{1}^{H}}{\partial t^{2}}+\left(\alpha I_{4}-\alpha^{2} I_{6}\right) \frac{\partial^{3} \psi_{z_{1}}^{H}}{\partial t^{2} \partial x_{1}}-\kappa\left(\frac{\partial^{2} v_{1}^{H}}{\partial x_{1}^{2}}-\frac{\partial \psi_{z_{1}}^{H}}{\partial x_{1}}\right)- \\
& \left(T+\tau_{0} S_{0}^{*}+\alpha^{2} J_{6}\right) \frac{\partial^{2} v_{1}^{H}}{\partial x_{1}^{2}}+\left(\alpha J_{4}-\alpha^{2} J_{6}\right) \frac{\partial^{3} \psi_{z_{1}}^{H}}{\partial x_{1}^{3}}+\left(\alpha^{2} J_{6}-\frac{2 \alpha \nu_{b} I_{4}^{\prime} \tau_{0}}{D_{0}}\right) \frac{\partial^{4} v_{1}^{H}}{\partial x_{1}^{4}} \\
& -B_{0} I_{0} \frac{\partial w_{1}^{H}}{\partial x_{1}}+\frac{\mu_{0} I_{0}^{2}}{4 \pi} \int_{0}^{l_{b}} \frac{\left(x_{2}-x_{1}\right) \frac{\partial v_{2}^{H}}{\partial x_{2}}-\left(v_{2}^{H}-v_{1}^{H}\right)}{\left(\left(x_{2}-x_{1}\right)^{2}+d^{2}\right)^{\frac{3}{2}}} \mathrm{~d} x_{2}=0, \\
& \left(I_{2}+I_{2}^{*}-2 \alpha\left(I_{4}+I_{4}^{*}\right)+\alpha^{2}\left(I_{6}+I_{6}^{*}\right)\right) \frac{\partial^{2} \psi_{y_{1}}^{H}}{\partial t^{2}}+\left(\alpha^{2}\left(I_{6}+I_{6}^{*}\right)-\alpha\left(I_{4}+I_{4}^{*}\right)\right) \frac{\partial^{3} w_{1}^{H}}{\partial t^{2} \partial x_{1}}- \\
& \left(\alpha J_{4}-\alpha^{2} J_{6}+\left(\lambda_{0}+2 \mu_{0}\right) \alpha I_{4}^{\prime *}-\frac{2 \nu_{b} \tau_{0}}{D_{0}}\left(I_{2}^{\prime}-\alpha I_{4}^{\prime}\right)\right) \frac{\partial^{3} w_{1}^{H}}{\partial x_{1}^{3}}-\left(J_{2}-2 \alpha J_{4}+\alpha^{2} J_{6}+\right. \\
& \left.\left(\lambda_{0}+2 \mu_{0}\right)\left(I_{2}^{\prime *}-\alpha I_{4}^{\prime *}\right)\right) \frac{\partial^{2} \psi_{y_{1}}^{H}}{\partial x_{1}^{2}}-\kappa\left(\frac{\partial w_{1}^{H}}{\partial x_{1}}-\psi_{y_{1}}^{H}\right)=0 \\
& \left(I_{0}+I_{0}^{*}+\frac{2 \alpha \nu_{b} I_{4}^{\prime} \rho_{0}}{D_{0}}\right) \frac{\partial^{2} w_{1}^{H}}{\partial t^{2}}+\left(\alpha I_{4}-\alpha^{2} I_{6}\right) \frac{\partial^{3} \psi_{y_{1}}^{H}}{\partial t^{2} \partial x_{1}}-\kappa\left(\frac{\partial^{2} w_{1}^{H}}{\partial x_{1}^{2}}-\frac{\partial \psi_{y_{1}}^{H}}{\partial x_{1}}\right)- \\
& \left(T+\tau_{0} S_{0}^{*}+\alpha^{2} J_{6}\right) \frac{\partial^{2} w_{1}^{H}}{\partial x_{1}^{2}}+\left(\alpha J_{4}-\alpha^{2} J_{6}\right) \frac{\partial^{3} \psi_{y_{1}}^{H}}{\partial x_{1}^{3}}+\left(\alpha^{2} J_{6}-\frac{2 \alpha \nu_{b} I_{4}^{\prime} \tau_{0}}{D_{0}}\right) \frac{\partial^{4} w_{1}^{H}}{\partial x_{1}^{4}}+ \\
& B_{0} I_{0} \frac{\partial v_{1}^{H}}{\partial x_{1}}+\frac{\mu_{0} I_{0}^{2}}{4 \pi} \int_{0}^{l_{b}} \frac{\left(x_{2}-x_{1}\right) \frac{\partial w_{2}^{H}}{\partial x_{2}}-\left(w_{2}^{H}-w_{1}^{H}\right)}{\left(\left(x_{2}-x_{1}\right)^{2}+d^{2}\right)^{\frac{3}{2}}} \mathrm{~d} x_{2}=0,
\end{aligned}
$$




$$
\begin{aligned}
& \left(I_{2}+I_{2}^{*}-2 \alpha\left(I_{4}+I_{4}^{*}\right)+\alpha^{2}\left(I_{6}+I_{6}^{*}\right)\right) \frac{\partial^{2} \psi_{z_{2}}^{H}}{\partial t^{2}}+\left(\alpha^{2}\left(I_{6}+I_{6}^{*}\right)-\alpha\left(I_{4}+I_{4}^{*}\right)\right) \frac{\partial^{3} v_{2}^{H}}{\partial t^{2} \partial x_{2}}- \\
& \left(\alpha J_{4}-\alpha^{2} J_{6}+\left(\lambda_{0}+2 \mu_{0}\right) \alpha I_{4}^{*}-\frac{2 \nu_{b} \tau_{0}}{D_{0}}\left(I_{2}^{\prime}-\alpha I_{4}^{\prime}\right)\right) \frac{\partial^{3} v_{2}^{H}}{\partial x_{2}^{3}}-\left(J_{2}-2 \alpha J_{4}+\alpha^{2} J_{6}+\right. \\
& \left.\left(\lambda_{0}+2 \mu_{0}\right)\left(I_{2}^{\prime *}-\alpha I_{4}^{\prime *}\right)\right) \frac{\partial^{2} \psi_{z_{2}}^{H}}{\partial x_{2}^{2}}-\kappa\left(\frac{\partial v_{2}^{H}}{\partial x_{2}}-\psi_{z_{2}}^{H}\right)=0 \\
& \left(I_{0}+I_{0}^{*}+\frac{2 \alpha \nu_{b} I_{4}^{\prime} \rho_{0}}{D_{0}}\right) \frac{\partial^{2} v_{2}^{H}}{\partial t^{2}}+\left(\alpha I_{4}-\alpha^{2} I_{6}\right) \frac{\partial^{3} \psi_{z_{2}}^{H}}{\partial t^{2} \partial x_{2}}-\kappa\left(\frac{\partial^{2} v_{2}^{H}}{\partial x_{2}^{2}}-\frac{\partial \psi_{z_{2}}^{H}}{\partial x_{2}}\right)- \\
& \left(T+\tau_{0} S_{0}^{*}+\alpha^{2} J_{6}\right) \frac{\partial^{2} v_{2}^{H}}{\partial x_{2}^{2}}+\left(\alpha J_{4}-\alpha^{2} J_{6}\right) \frac{\partial^{3} \psi_{z_{2}}^{H}}{\partial x_{2}^{3}}+\left(\alpha^{2} J_{6}-\frac{2 \alpha \nu_{b} I_{4}^{\prime} \tau_{0}}{D_{0}}\right) \frac{\partial^{4} v_{2}^{H}}{\partial x_{2}^{4}} \\
& -B_{0} I_{0} \frac{\partial w_{2}^{H}}{\partial x_{2}}-\frac{\mu_{0} I_{0}^{2}}{4 \pi} \int_{0}^{l_{b}} \frac{\left(x_{2}-x_{1}\right) \frac{\partial v_{1}^{H}}{\partial x_{1}}-\left(v_{2}^{H}-v_{1}^{H}\right)}{\left(\left(x_{2}-x_{1}\right)^{2}+d^{2}\right)^{\frac{3}{2}}} \mathrm{~d} x_{1}=0, \\
& \left(I_{2}+I_{2}^{*}-2 \alpha\left(I_{4}+I_{4}^{*}\right)+\alpha^{2}\left(I_{6}+I_{6}^{*}\right)\right) \frac{\partial^{2} \psi_{y_{2}}^{H}}{\partial t^{2}}+\left(\alpha^{2}\left(I_{6}+I_{6}^{*}\right)-\alpha\left(I_{4}+I_{4}^{*}\right)\right) \frac{\partial^{3} w_{2}^{H}}{\partial t^{2} \partial x_{2}}- \\
& \left(\alpha J_{4}-\alpha^{2} J_{6}+\left(\lambda_{0}+2 \mu_{0}\right) \alpha I_{4}^{* *}-\frac{2 \nu_{b} \tau_{0}}{D_{0}}\left(I_{2}^{\prime}-\alpha I_{4}^{\prime}\right)\right) \frac{\partial^{3} w_{2}^{H}}{\partial x_{2}^{3}}-\left(J_{2}-2 \alpha J_{4}+\alpha^{2} J_{6}+\right. \\
& \left.\left(\lambda_{0}+2 \mu_{0}\right)\left(I_{2}^{\prime *}-\alpha I_{4}^{\prime *}\right)\right) \frac{\partial^{2} \psi_{y_{2}}^{H}}{\partial x_{2}^{2}}-\kappa\left(\frac{\partial w_{2}^{H}}{\partial x_{2}}-\psi_{y_{2}}^{H}\right)=0 \\
& \left(I_{0}+I_{0}^{*}+\frac{2 \alpha \nu_{b} I_{4}^{\prime} \rho_{0}}{D_{0}}\right) \frac{\partial^{2} w_{2}^{H}}{\partial t^{2}}+\left(\alpha I_{4}-\alpha^{2} I_{6}\right) \frac{\partial^{3} \psi_{y_{2}}^{H}}{\partial t^{2} \partial x_{2}}-\kappa\left(\frac{\partial^{2} w_{2}^{H}}{\partial x_{2}^{2}}-\frac{\partial \psi_{y_{2}}^{H}}{\partial x_{2}}\right)- \\
& \left(T+\tau_{0} S_{0}^{*}+\alpha^{2} J_{6}\right) \frac{\partial^{2} w_{2}^{H}}{\partial x_{2}^{2}}+\left(\alpha J_{4}-\alpha^{2} J_{6}\right) \frac{\partial^{3} \psi_{y_{2}}^{H}}{\partial x_{2}^{3}}+\left(\alpha^{2} J_{6}-\frac{2 \alpha \nu_{b} I_{4}^{\prime} \tau_{0}}{D_{0}}\right) \frac{\partial^{4} w_{2}^{H}}{\partial x_{2}^{4}}+ \\
& B_{0} I_{0} \frac{\partial v_{2}^{H}}{\partial x_{2}}+\frac{\mu_{0} I_{0}^{2}}{4 \pi} \int_{0}^{l_{b}} \frac{\left(x_{2}-x_{1}\right) \frac{\partial w_{1}^{H}}{\partial x_{1}}-\left(w_{2}^{H}-w_{1}^{H}\right)}{\left(\left(x_{2}-x_{1}\right)^{2}+d^{2}\right)^{\frac{3}{2}}} \mathrm{~d} x_{1}=0 .
\end{aligned}
$$

By introducing the dimensionless parameters in Eq. (59) to Eqs. (61a)-(61h), the dimensionless equations of motion of magnetically affected DCCNWs according to the exact version of the mutually exerted magnetic force and the hypotheses of the HOBT are obtained:

$$
\begin{aligned}
& \left(1+\chi_{1}^{H}\right) \frac{\partial^{2} \bar{\psi}_{z_{1}}^{H}}{\partial \tau^{2}}+\gamma_{6}^{2}\left(1+\chi_{2}^{H}\right) \frac{\partial^{3} \bar{v}_{1}^{H}}{\partial \tau^{2} \partial \xi_{1}}-\gamma_{9}^{2}\left(1+\chi_{3}^{H}\right) \frac{\partial^{3} \bar{v}_{1}^{H}}{\partial \xi_{1}^{3}} \\
& -\gamma_{8}^{2}\left(1+\chi_{4}^{H}\right) \frac{\partial^{2} \bar{\psi}_{z_{1}}^{H}}{\partial \xi_{1}^{2}}-\gamma_{7}^{2}\left(\frac{\partial \bar{v}_{1}^{H}}{\partial \xi_{1}}-\bar{\psi}_{z_{1}}^{H}\right)=0
\end{aligned}
$$




$$
\begin{aligned}
& \left(1+\chi_{5}^{H}\right) \frac{\partial^{2} \bar{v}_{1}^{H}}{\partial \tau^{2}}-\gamma_{2}^{2} \frac{\partial^{4} \bar{v}_{1}^{H}}{\partial \tau^{2} \partial \xi_{1}^{2}}+\gamma_{1}^{2} \frac{\partial^{3} \bar{\psi}_{z_{1}}^{H}}{\partial \tau^{2} \partial \xi_{1}}-\gamma_{3}^{2}\left(\frac{\partial^{2} \bar{v}_{1}^{H}}{\partial \xi_{1}^{2}}-\frac{\partial \bar{\psi}_{z_{1}}^{H}}{\partial \xi_{1}}\right) \\
& -\left(\bar{T}^{H}+\chi_{6}^{H}\right) \frac{\partial^{2} \bar{v}_{1}^{H}}{\partial \xi_{1}^{2}}+\gamma_{4}^{2} \frac{\partial^{3} \bar{\psi}_{z_{1}}^{H}}{\partial \xi_{1}^{3}}+\left(1-\chi_{7}^{H}\right) \frac{\partial^{4} \bar{v}_{1}^{H}}{\partial \xi_{1}^{4}}-\bar{f}_{0}^{H} \frac{\partial \bar{w}_{1}^{H}}{\partial \xi_{1}} \\
& +\frac{\bar{f}_{1}^{H}}{2 \bar{d}} \int_{0}^{1} \frac{\left(\xi_{2}-\xi_{1}\right) \frac{\partial \bar{v}_{2}^{H}}{\partial \xi_{2}}-\left(\bar{v}_{2}^{H}-\bar{v}_{1}^{H}\right)}{\left(\left(\frac{\xi_{2}-\xi_{1}}{\bar{d}}\right)^{2}+1\right)^{\frac{3}{2}}} \mathrm{~d} \xi_{2}=0, \\
& \left(1+\chi_{1}^{H}\right) \frac{\partial^{2} \bar{\psi}_{y_{1}}^{H}}{\partial \tau^{2}}+\gamma_{6}^{2}\left(1+\chi_{2}^{H}\right) \frac{\partial^{3} \bar{w}_{1}^{H}}{\partial \tau^{2} \partial \xi_{1}}-\gamma_{9}^{2}\left(1+\chi_{3}^{H}\right) \frac{\partial^{3} \bar{w}_{1}^{H}}{\partial \xi_{1}^{3}} \\
& -\gamma_{8}^{2}\left(1+\chi_{4}^{H}\right) \frac{\partial^{2} \bar{\psi}_{y_{1}}^{H}}{\partial \xi_{1}^{2}}-\gamma_{7}^{2}\left(\frac{\partial \bar{w}_{1}^{H}}{\partial \xi_{1}}-\bar{\psi}_{y_{1}}^{H}\right)=0, \\
& \left(1+\chi_{5}^{H}\right) \frac{\partial^{2} \bar{w}_{1}^{H}}{\partial \tau^{2}}-\gamma_{2}^{2} \frac{\partial^{4} \bar{w}_{1}^{H}}{\partial \tau^{2} \partial \xi_{1}^{2}}+\gamma_{1}^{2} \frac{\partial^{3} \bar{\psi}_{y_{1}}^{H}}{\partial \tau^{2} \partial \xi_{1}}-\gamma_{3}^{2}\left(\frac{\partial^{2} \bar{w}_{1}^{H}}{\partial \xi_{1}^{2}}-\frac{\partial \bar{\psi}_{y_{1}}^{H}}{\partial \xi_{1}}\right) \\
& -\left(\bar{T}^{H}+\chi_{6}^{H}\right) \frac{\partial^{2} \bar{w}_{1}^{H}}{\partial \xi_{1}^{2}}+\gamma_{4}^{2} \frac{\partial^{3} \bar{\psi}_{y_{1}}^{H}}{\partial \xi_{1}^{3}}+\left(1-\chi_{7}^{H}\right) \frac{\partial^{4} \bar{w}_{1}^{H}}{\partial \xi_{1}^{4}}+\bar{f}_{0}^{H} \frac{\partial \bar{v}_{1}^{H}}{\partial \xi_{1}} \\
& +\frac{\bar{f}_{1}^{H}}{2 \bar{d}} \int_{0}^{1} \frac{\left(\xi_{2}-\xi_{1}\right) \frac{\partial \bar{w}_{2}^{H}}{\partial \xi_{2}}-\left(\bar{w}_{2}^{H}-\bar{w}_{1}^{H}\right)}{\left(\left(\frac{\xi_{2}-\xi_{1}}{\bar{d}}\right)^{2}+1\right)^{\frac{3}{2}}} \mathrm{~d} \xi_{2}=0 \\
& \left(1+\chi_{1}^{H}\right) \frac{\partial^{2} \bar{\psi}_{z_{2}}^{H}}{\partial \tau^{2}}+\gamma_{6}^{2}\left(1+\chi_{2}^{H}\right) \frac{\partial^{3} \bar{v}_{2}^{H}}{\partial \tau^{2} \partial \xi_{2}}-\gamma_{9}^{2}\left(1+\chi_{3}^{H}\right) \frac{\partial^{3} \bar{v}_{2}^{H}}{\partial \xi_{2}^{3}} \\
& -\gamma_{8}^{2}\left(1+\chi_{4}^{H}\right) \frac{\partial^{2} \bar{\psi}_{z_{2}}^{H}}{\partial \xi_{2}^{2}}-\gamma_{7}^{2}\left(\frac{\partial \bar{v}_{2}^{H}}{\partial \xi_{2}}-\bar{\psi}_{z_{2}}^{H}\right)=0, \\
& \left(1+\chi_{5}^{H}\right) \frac{\partial^{2} \bar{v}_{2}^{H}}{\partial \tau^{2}}-\gamma_{2}^{2} \frac{\partial^{4} \bar{v}_{2}^{H}}{\partial \tau^{2} \partial \xi_{2}^{2}}+\gamma_{1}^{2} \frac{\partial^{3} \bar{\psi}_{z_{2}}^{H}}{\partial \tau^{2} \partial \xi_{2}}-\gamma_{3}^{2}\left(\frac{\partial^{2} \bar{v}_{2}^{H}}{\partial \xi_{2}^{2}}-\frac{\partial \bar{\psi}_{z_{2}}^{H}}{\partial \xi_{2}}\right) \\
& -\left(\bar{T}^{H}+\chi_{6}^{H}\right) \frac{\partial^{2} \bar{v}_{2}^{H}}{\partial \xi_{2}^{2}}+\gamma_{4}^{2} \frac{\partial^{3} \bar{\psi}_{z_{2}}^{H}}{\partial \xi_{2}^{3}}+\left(1-\chi_{7}^{H}\right) \frac{\partial^{4} \bar{v}_{2}^{H}}{\partial \xi_{2}^{4}}-\bar{f}_{0}^{H} \frac{\partial \bar{w}_{2}^{H}}{\partial \xi_{2}} \\
& -\frac{\bar{f}_{1}^{H}}{2 \bar{d}} \int_{0}^{1} \frac{\left(\xi_{2}-\xi_{1}\right) \frac{\partial \bar{v}_{1}^{H}}{\partial \xi_{1}}-\left(\bar{v}_{2}^{H}-\bar{v}_{1}^{H}\right)}{\left(\left(\frac{\xi_{2}-\xi_{1}}{\bar{d}}\right)^{2}+1\right)^{\frac{3}{2}}} \mathrm{~d} \xi_{1}=0,
\end{aligned}
$$




$$
\begin{gathered}
\left(1+\chi_{1}^{H}\right) \frac{\partial^{2} \bar{\psi}_{y_{2}}^{H}}{\partial \tau^{2}}+\gamma_{6}^{2}\left(1+\chi_{2}^{H}\right) \frac{\partial^{3} \bar{w}_{2}^{H}}{\partial \tau^{2} \partial \xi_{2}}-\gamma_{9}^{2}\left(1+\chi_{3}^{H}\right) \frac{\partial^{3} \bar{w}_{2}^{H}}{\partial \xi_{2}^{3}} \\
-\gamma_{8}^{2}\left(1+\chi_{4}^{H}\right) \frac{\partial^{2} \bar{\psi}_{y_{2}}^{H}}{\partial \xi_{2}^{2}}-\gamma_{7}^{2}\left(\frac{\partial \bar{w}_{2}^{H}}{\partial \xi_{2}}-\bar{\psi}_{y_{2}}^{H}\right)=0, \\
\left(1+\chi_{5}^{H}\right) \frac{\partial^{2} \bar{w}_{2}^{H}}{\partial \tau^{2}}-\gamma_{2}^{2} \frac{\partial^{4} \bar{w}_{2}^{H}}{\partial \tau^{2} \partial \xi_{2}^{2}}+\gamma_{1}^{2} \frac{\partial^{3} \bar{\psi}_{y_{2}}^{H}}{\partial \tau^{2} \partial \xi_{2}}-\gamma_{3}^{2}\left(\frac{\partial^{2} \bar{w}_{2}^{H}}{\partial \xi_{2}^{2}}-\frac{\partial \bar{\psi}_{y_{2}}^{H}}{\partial \xi_{2}}\right) \\
-\left(\bar{T}^{H}+\chi_{6}^{H}\right) \frac{\partial^{2} \bar{w}_{2}^{H}}{\partial \xi_{2}^{2}}+\gamma_{4}^{2} \frac{\partial^{3} \bar{\psi}_{y_{2}}^{H}}{\partial \xi_{2}^{3}}+\left(1-\chi_{7}^{H}\right) \frac{\partial^{4} \bar{w}_{2}^{H}}{\partial \xi_{2}^{4}}+\bar{f}_{0}^{H} \frac{\partial \bar{v}_{2}^{H}}{\partial \xi_{2}} \\
-\frac{\bar{f}_{1}^{H}}{2 \bar{d}} \int_{0}^{1} \frac{\left(\xi_{2}-\xi_{1}\right) \frac{\partial \bar{w}_{1}^{H}}{\partial \xi_{1}}-\left(\bar{w}_{2}^{H}-\bar{w}_{1}^{H}\right)}{\left(\left(\frac{\xi_{2}-\xi_{1}}{\bar{d}}\right)^{2}+1\right)^{\frac{3}{2}} \mathrm{~d} \xi_{1}=0 .}
\end{gathered}
$$

6.3. Vibration of the magnetically affected nanosystem based on the HOBT via RKPM

\subsubsection{Free transverse vibration on the basis of the proposed approximate model}

Let us to discretize the deformation fields of the magnetically affected DCCNWs on the basis of the HOBT as follows:

$$
\begin{aligned}
& \bar{v}_{i}^{H}\left(\xi_{i}, \tau\right)=\sum_{I=1}^{N P_{i}} \phi_{I}^{v_{i}}\left(\xi_{i}\right) \bar{v}_{i_{I}}^{H}(\tau), \quad \bar{\psi}_{z_{i}}^{H}\left(\xi_{i}, \tau\right)=\sum_{I=1}^{N P_{i}} \phi_{I}^{\psi_{z_{i}}}\left(\xi_{i}\right) \bar{\psi}_{z_{i_{I}}}^{H}(\tau), \\
& \bar{w}_{i}^{H}\left(\xi_{i}, \tau\right)=\sum_{I=1}^{N P_{i}} \phi_{I}^{w_{i}}\left(\xi_{i}\right) \bar{w}_{i_{I}}^{H}(\tau), \quad \bar{\psi}_{y_{i}}^{H}\left(\xi_{i}, \tau\right)=\sum_{I=1}^{N P_{i}} \phi_{I}^{\psi_{y_{i}}}\left(\xi_{i}\right) \bar{\psi}_{y_{i_{I}}}^{H}(\tau),
\end{aligned}
$$

where $\phi_{I}^{v_{i}}, \phi_{I}^{w_{i}}, \phi_{I}^{\psi_{z_{i}}}$, and $\phi_{I}^{\psi_{y_{i}}}$ denote the shape functions of the $I$ th particle of RKPM which are pertinent to the dimensionless deformation fields $\bar{v}_{i}^{H}, \bar{w}_{i}^{H}, \bar{\psi}_{z_{i}}^{H}$, and $\bar{\psi}_{y_{i}}^{H}$, respectively. Further, $\bar{v}_{i_{I}}^{T}, \bar{w}_{i_{I}}^{T}, \bar{\psi}_{z_{i_{I}}}^{T}$, and $\bar{\psi}_{y_{i_{I}}}^{T}$ represent their corresponding dimensionless nodal parameter values. In order to arrive at the weak form of the given dimensionless equations of motion via RKPM, Eqs. (60a)-(60h) are premultiplied by $\delta \bar{\psi}_{z_{1}}^{H}, \delta \bar{v}_{1}^{H}, \delta \bar{\psi}_{y_{1}}^{H}, \delta \bar{w}_{1}^{H}, \delta \bar{\psi}_{z_{2}}^{H}, \delta \bar{v}_{2}^{H}$, $\delta \bar{\psi}_{y_{2}}^{H}$, and $\delta \bar{w}_{2}^{H}$, respectively, and the resulted relations are integrated over the dimensionless longitudinal domain of the CCNWs. After taking the successful integration by parts, and using the discretized dimensionless deformation fields in Eq. (63), the following set of 
second-order ODEs is obtainable:

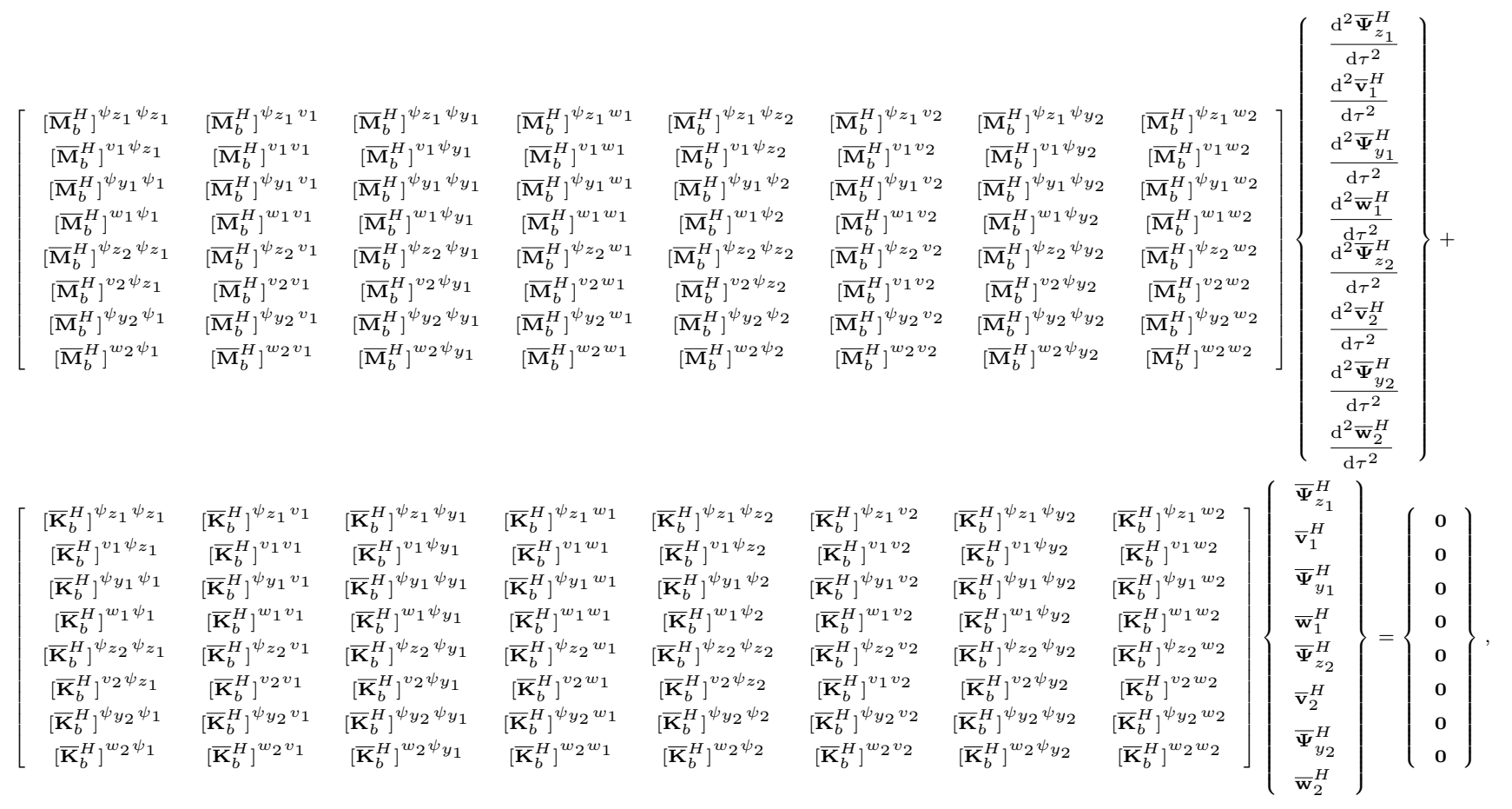

(64)

where the nonzero dimensionless mass and stiffness submatrices as well as the time dependent vectors are evaluated as:

$$
\begin{gathered}
{\left[\overline{\mathbf{M}}_{b}^{H}\right]_{I J}^{\psi_{z_{i}} \psi_{z_{i}}}=\int_{0}^{1}\left(1+\chi_{1}^{H}\right) \phi_{I}^{\psi_{z_{i}}} \phi_{J}^{\psi_{z_{i}}} \mathrm{~d} \xi_{i},} \\
{\left[\overline{\mathbf{M}}_{b}^{H}\right]_{I J}^{\psi_{z_{i}} v_{i}}=\int_{0}^{1} \gamma_{6}^{2}\left(1+\chi_{2}^{H}\right) \phi_{I}^{\psi_{z_{i}}} \frac{\mathrm{d} \phi_{J}^{v}}{\mathrm{~d} \xi_{i}} \mathrm{~d} \xi_{i},} \\
{\left[\overline{\mathbf{M}}_{b}^{H}\right]_{I J}^{v_{i} \psi_{z_{i}}}=-\int_{0}^{1} \gamma_{1}^{2} \frac{\mathrm{d} \phi_{I}^{v_{i}}}{\mathrm{~d} \xi_{i}} \phi_{J}^{\psi_{z_{i}}} \mathrm{~d} \xi_{i}} \\
{\left[\overline{\mathbf{M}}_{b}^{H}\right]_{I J}^{v_{i} v_{i}}=\int_{0}^{1}\left(\left(1+\chi_{5}^{H}\right) \phi_{I}^{v} \phi_{J}^{v}+\gamma_{2}^{2} \frac{\mathrm{d} \phi_{I}^{v}}{\mathrm{~d} \xi_{i}} \frac{\mathrm{d} \phi_{J}^{v}}{\mathrm{~d} \xi_{i}}\right) \mathrm{d} \xi_{i},} \\
{\left[\overline{\mathbf{M}}_{b}^{H}\right]_{I J}^{\psi_{y_{i}} \psi_{y_{i}}}=\int_{0}^{1}\left(1+\chi_{1}^{H}\right) \phi_{I}^{\psi_{y_{i}}} \phi_{J}^{\psi_{y_{i}}} \mathrm{~d} \xi_{i},} \\
{\left[\overline{\mathbf{M}}_{b}^{H}\right]_{I J}^{\psi_{y_{i} w_{i}}}=\int_{0}^{1} \gamma_{6}^{2}\left(1+\chi_{2}^{H}\right) \phi_{I}^{\psi_{y_{i}}} \frac{\mathrm{d} \phi_{J}^{w_{i}}}{\mathrm{~d} \xi_{i}} \mathrm{~d} \xi_{i},}
\end{gathered}
$$




$$
\begin{aligned}
& {\left[\overline{\mathbf{M}}_{b}^{H}\right]_{I J}^{w_{i} \psi_{y_{i}}}=-\int_{0}^{1} \gamma_{1}^{2} \frac{\mathrm{d} \phi_{I}^{w_{i}}}{\mathrm{~d} \xi_{i}} \phi_{J}^{\psi_{y_{i}}} \mathrm{~d} \xi_{i}} \\
& {\left[\overline{\mathbf{M}}_{b}^{H}\right]_{I J}^{w_{i} w_{i}}=\int_{0}^{1}\left(\left(1+\chi_{5}^{H}\right) \phi_{I}^{w_{i}} \phi_{J}^{w_{i}}+\gamma_{2}^{2} \frac{\mathrm{d} \phi_{I}^{w_{i}}}{\mathrm{~d} \xi_{i}} \frac{\mathrm{d} \phi_{J}^{w_{i}}}{\mathrm{~d} \xi_{i}}\right) \mathrm{d} \xi_{i}} \\
& {\left[\overline{\mathbf{K}}_{b}^{H}\right]_{I J}^{\psi_{z_{i}} \psi_{z_{i}}}=\int_{0}^{1}\left(\gamma_{7}^{2} \phi_{I}^{\psi_{z_{i}}} \phi_{J}^{\psi_{z_{i}}}+\gamma_{8}^{2}\left(1+\chi_{4}^{H}\right) \frac{\mathrm{d} \phi_{I}^{\psi_{z_{i}}}}{\mathrm{~d} \xi_{i}} \frac{\mathrm{d} \phi_{J}^{\psi_{z_{i}}}}{\mathrm{~d} \xi_{i}}\right) \mathrm{d} \xi_{i},} \\
& {\left[\overline{\mathbf{K}}_{b}^{H}\right]_{I J}^{\psi_{z_{i}} v_{i}}=-\int_{0}^{1}\left(\gamma_{7}^{2} \phi_{I}^{\psi_{z_{i}}} \frac{\mathrm{d} \phi_{J}^{v_{i}}}{\mathrm{~d} \xi_{i}}-\gamma_{9}^{2}\left(1+\chi_{3}^{H}\right) \frac{\mathrm{d} \phi_{I}^{\psi_{z_{i}}}}{\mathrm{~d} \xi_{i}} \frac{\mathrm{d}^{2} \phi_{J}^{v_{i}}}{\mathrm{~d} \xi_{i}^{2}}\right) \mathrm{d} \xi_{i},} \\
& {\left[\overline{\mathbf{K}}_{b}^{H}\right]_{I J}^{v_{i} \psi_{z_{i}}}=-\int_{0}^{1}\left(\gamma_{3}^{2} \frac{\mathrm{d} \phi_{I}^{v_{i}}}{\mathrm{~d} \xi_{i}} \phi_{J}^{\psi_{z_{i}}}-\gamma_{4}^{2} \frac{\mathrm{d}^{2} \phi_{I}^{v_{i}}}{\mathrm{~d} \xi_{i}^{2}} \frac{\mathrm{d} \phi_{J}^{\psi_{z_{i}}}}{\mathrm{~d} \xi_{i}}\right) \mathrm{d} \xi_{i}} \\
& {\left[\overline{\mathbf{K}}_{b}^{H}\right]_{I J}^{v_{i} v_{i}}=\int_{0}^{1}\left(\left(\gamma_{3}^{2}+\bar{T}^{H}+\chi_{6}^{H}\right) \frac{\mathrm{d} \phi_{I}^{v_{i}}}{\mathrm{~d} \xi_{i}} \frac{\mathrm{d} \phi_{J}^{v_{i}}}{\mathrm{~d} \xi_{i}}+\left(1-\chi_{7}^{H}\right) \frac{\mathrm{d}^{2} \phi_{I}^{v_{i}}}{\mathrm{~d} \xi_{i}^{2}} \frac{\mathrm{d}^{2} \phi_{J}^{v_{i}}}{\mathrm{~d} \xi_{i}^{2}}-\bar{f}_{1}^{H} \phi_{I}^{v_{i}} \phi_{J}^{v_{i}}\right) \mathrm{d} \xi_{i},} \\
& {\left[\overline{\mathbf{K}}_{b}^{H}\right]_{I J}^{v_{i} v_{j}}=\int_{0}^{1} \bar{f}_{1}^{H} \phi_{I}^{v_{i}} \phi_{J}^{v_{j}} \mathrm{~d} \xi_{j} ; i \neq j} \\
& {\left[\overline{\mathbf{K}}_{b}^{H}\right]_{I J}^{v_{i} w_{i}}=-\int_{0}^{1} \bar{f}_{0}^{H} \phi_{I}^{v_{i}} \frac{\mathrm{d} \phi_{J}^{w_{i}}}{\mathrm{~d} \xi_{i}} \mathrm{~d} \xi_{i}} \\
& {\left[\overline{\mathbf{K}}_{b}^{H}\right]_{I J}^{\psi_{y_{i}} \psi_{y_{i}}}=\int_{0}^{1}\left(\gamma_{7}^{2} \phi_{I}^{\psi_{y_{i}}} \phi_{J}^{\psi_{y_{i}}}+\gamma_{8}^{2}\left(1+\chi_{4}^{H}\right) \frac{\mathrm{d} \phi_{I}^{\psi_{y_{i}}}}{\mathrm{~d} \xi_{i}} \frac{\mathrm{d} \phi_{J}^{\psi_{y_{i}}}}{\mathrm{~d} \xi_{i}}\right) \mathrm{d} \xi_{i}} \\
& {\left[\overline{\mathbf{K}}_{b}^{H}\right]_{I J}^{\psi_{y_{i} w_{i}}}=-\int_{0}^{1}\left(\gamma_{7}^{2} \phi_{I}^{\psi_{y_{i}}} \frac{\mathrm{d} \phi_{J}^{w_{i}}}{\mathrm{~d} \xi_{i}}-\gamma_{9}^{2}\left(1+\chi_{3}^{H}\right) \frac{\mathrm{d} \phi_{I}^{\psi_{y_{i}}}}{\mathrm{~d} \xi_{i}} \frac{\mathrm{d}^{2} \phi_{J}^{w_{i}}}{\mathrm{~d} \xi_{i}^{2}}\right) \mathrm{d} \xi_{i}} \\
& {\left[\overline{\mathbf{K}}_{b}^{H}\right]_{I J}^{w_{i} \psi_{y_{i}}}=-\int_{0}^{1}\left(\gamma_{3}^{2} \frac{\mathrm{d} \phi_{I}^{w_{i}}}{\mathrm{~d} \xi_{i}} \phi_{J}^{\psi_{y_{i}}}-\gamma_{4}^{2} \frac{\mathrm{d}^{2} \phi_{I}^{w_{i}}}{\mathrm{~d} \xi_{i}^{2}} \frac{\mathrm{d} \phi_{J}^{\psi_{y_{i}}}}{\mathrm{~d} \xi_{i}}\right) \mathrm{d} \xi_{i}} \\
& {\left[\overline{\mathbf{K}}_{b}^{H}\right]_{I J}^{w_{i} w_{i}}=\int_{0}^{1}\left(\left(\gamma_{3}^{2}+\bar{T}^{H}+\chi_{6}^{H}\right) \frac{\mathrm{d} \phi_{I}^{w_{i}}}{\mathrm{~d} \xi_{i}} \frac{\mathrm{d} \phi_{J}^{w_{i}}}{\mathrm{~d} \xi_{i}}+\left(1-\chi_{7}^{H}\right) \frac{\mathrm{d}^{2} \phi_{I}^{w_{i}}}{\mathrm{~d} \xi_{i}^{2}} \frac{\mathrm{d}^{2} \phi_{J}^{w_{i}}}{\mathrm{~d} \xi_{i}^{2}}+\bar{f}_{1}^{H} \phi_{I}^{w_{i}} \phi_{J}^{w_{i}}\right) \mathrm{d} \xi_{i},(65 \mathrm{r})}
\end{aligned}
$$




$$
\begin{gathered}
{\left[\overline{\mathbf{K}}_{b}^{H}\right]_{I J}^{w_{i} w_{j}}=-\int_{0}^{1} \bar{f}_{1}^{H} \phi_{I}^{w_{i}} \phi_{J}^{w_{j}} \mathrm{~d} \xi_{j} ; i \neq j} \\
{\left[\overline{\mathbf{K}}_{b}^{H}\right]_{I J}^{w_{i} v_{i}}=\int_{0}^{1} \bar{f}_{0}^{H} \phi_{I}^{w_{i}} \frac{\mathrm{d} \phi_{J}^{v_{i}}}{\mathrm{~d} \xi_{i}} \mathrm{~d} \xi_{i}} \\
\overline{\mathbf{\Psi}}_{z_{i}}^{H}=<\bar{\psi}_{z_{i 1}}^{H}, \bar{\psi}_{z_{i 2}}^{H}, \ldots, \bar{\psi}_{z_{i N P_{i}}}^{H}>^{\mathrm{T}} \\
\overline{\mathbf{v}}_{i}^{H}=<\bar{v}_{i 1}^{H}, \bar{v}_{i 2}^{H}, \ldots, \bar{v}_{i N P_{i}}^{H}>^{\mathrm{T}} \\
\overline{\mathbf{\Psi}}_{i_{y}}^{H}=<\bar{\psi}_{y_{i 1}}^{H}, \bar{\psi}_{y_{i 2}}^{H}, \ldots, \bar{\psi}_{y_{i N P_{i}}}^{H}>^{\mathrm{T}} \\
\overline{\mathbf{w}}_{i}^{H}=<\bar{w}_{i 1}^{H}, \bar{w}_{i 2}^{H}, \ldots, \bar{w}_{i N P_{i}}^{H}>^{\mathrm{T}} .
\end{gathered}
$$

\subsubsection{Free transverse vibration on the basis of the proposed exact model}

In order to construct the weak version of the strong form of governing equations based on the exact HOBT, we follow the given procedure in the previous part. For this purpose, Eqs. (62a)-(62h) in order are multiplied by $\delta \bar{\psi}_{z_{1}}^{H}, \delta \bar{v}_{1}^{H}, \delta \bar{\psi}_{y_{1}}^{H}, \delta \bar{w}_{1}^{H}, \delta \bar{\psi}_{z_{2}}^{H}, \delta \bar{v}_{2}^{H}, \delta \bar{\psi}_{y_{2}}^{H}$, and $\delta \bar{w}_{2}^{H}$, and the resulting equations are integrated over $[0,1]$. By taking the required integration by parts through exploiting Eq. (63), a set of ODEs as given in Eq. (64) is derived. All the mass submatrices as well as most of the stiffness submatrices of the exact version are identical to their counterparts of the suggested approximate model; nevertheless, some of the stiffness submatrices are modified as follows:

$$
\begin{aligned}
& {\left[\overline{\mathbf{K}}_{b}^{H}\right]_{I J}^{v_{i} v_{i}}=} \int_{0}^{1}\left(\left(\gamma_{3}^{2}+\bar{T}^{H}+\chi_{6}^{H}\right) \frac{\mathrm{d} \phi_{I}^{v_{i}}}{\mathrm{~d} \xi_{i}} \frac{\mathrm{d} \phi_{J}^{v_{i}}}{\mathrm{~d} \xi_{i}}+\left(1-\chi_{7}^{H}\right) \frac{\mathrm{d}^{2} \phi_{I}^{v_{i}}}{\mathrm{~d} \xi_{i}^{2}} \frac{\mathrm{d}^{2} \phi_{J}^{v_{i}}}{\mathrm{~d} \xi_{i}^{2}}\right) \mathrm{d} \xi_{i}+ \\
&+\frac{\bar{f}_{1}^{H}}{2 \bar{d}} \int_{0}^{1} \int_{0}^{1} \frac{\phi_{I}^{v_{i}}\left(\xi_{i}\right) \phi_{J}^{v_{i}}\left(\xi_{i}\right)}{\left(\left(\frac{\xi_{2}-\xi_{1}}{\bar{d}}\right)^{2}+1\right)^{\frac{3}{2}} \mathrm{~d} \xi_{2} \mathrm{~d} \xi_{1},} \\
& {\left[\overline{\mathbf{K}}_{b}^{H}\right]_{I J}^{v_{i} v_{j}}=-\frac{\bar{f}_{1}^{H}}{2 \bar{d}} \int_{0}^{1} \int_{0}^{1} \frac{\phi_{I}^{v_{i}}\left(\left(\xi_{i}-\xi_{j}\right) \frac{\mathrm{d} \phi_{J}^{v_{j}}}{\mathrm{~d} \xi_{j}}+\phi_{J}^{v_{j}}\right)}{\left(\left(\frac{\xi_{2}-\xi_{1}}{\bar{d}}\right)^{2}+1\right)^{\frac{3}{2}}} \mathrm{~d} \xi_{i} \mathrm{~d} \xi_{j} ; i \neq j, }
\end{aligned}
$$




$$
\begin{aligned}
{\left[\overline{\mathbf{K}}_{b}^{H}\right]_{I J}^{w_{i} w_{i}} } & =\int_{0}^{1}\left(\left(\gamma_{3}^{2}+\bar{T}^{H}+\chi_{6}^{H}\right) \frac{\mathrm{d} \phi_{I}^{w_{i}}}{\mathrm{~d} \xi_{i}} \frac{\mathrm{d} \phi_{J}^{w_{i}}}{\mathrm{~d} \xi_{i}}+\left(1-\chi_{7}^{H}\right) \frac{\mathrm{d}^{2} \phi_{I}^{w_{i}}}{\mathrm{~d} \xi_{i}^{2}} \frac{\mathrm{d}^{2} \phi_{J}^{w_{i}}}{\mathrm{~d} \xi_{i}^{2}}\right) \mathrm{d} \xi_{i}+ \\
& +\frac{\bar{f}_{1}^{H}}{2 \bar{d}} \int_{0}^{1} \int_{0}^{1} \frac{\phi_{I}^{w_{i}}\left(\xi_{i}\right) \phi_{J}^{w_{i}}\left(\xi_{i}\right)}{\left(\left(\frac{\xi_{2}-\xi_{1}}{\bar{d}}\right)^{2}+1\right)^{\frac{3}{2}} \mathrm{~d} \xi_{2} \mathrm{~d} \xi_{1},} \\
{\left[\overline{\mathbf{K}}_{b}^{H}\right]_{I J}^{w_{i} w_{j}} } & =-\frac{\bar{f}_{1}^{H}}{2 \bar{d}} \int_{0}^{1} \int_{0}^{1} \frac{\phi_{I}^{w_{i}}\left(\left(\xi_{i}-\xi_{j}\right) \frac{\mathrm{d} \phi_{J}^{w_{j}}}{\mathrm{~d} \xi_{j}}+\phi_{J}^{w_{j}}\right)}{\left(\left(\frac{\xi_{2}-\xi_{1}}{\bar{d}}\right)^{2}+1\right)^{\frac{3}{2}}} \mathrm{~d} \xi_{i} \mathrm{~d} \xi_{j} ; i \neq j .
\end{aligned}
$$

\subsubsection{Enforcement of boundary conditions and frequency analysis}

For simply supported DCCNWs which are modeled by the HOBT, the following boundary conditions should be satisfied:

$$
\begin{aligned}
& \bar{w}_{i}^{H}(0, \tau)=\bar{w}_{i}^{H}(1, \tau)=0 ; \quad \bar{M}_{b_{y i}}^{H}(0, \tau)=\bar{M}_{b_{y i}}^{H}(1, \tau)=0, \\
& \bar{v}_{i}^{H}(0, \tau)=\bar{v}_{i}^{H}(1, \tau)=0 ; \quad \bar{M}_{b_{z i}}^{H}(0, \tau)=\bar{M}_{b_{z i}}^{H}(1, \tau)=0
\end{aligned}
$$

where $\bar{M}_{b_{y i}}^{H}=\frac{M_{b_{y i}} l_{b}}{\alpha^{2} J_{6}}$ and $\bar{M}_{b_{z i}}^{H}=\frac{M_{b_{z i}} l_{b}}{\alpha^{2} J_{6}}$. To impose the essential conditions in Eq. (67), the CCA is implemented. By assuming a harmonic form for the time-dependent vectors and by solving the resulting set of eigenvalue equations for the dimensionless frequencies, the free dynamic response of the nanosystem could be readily investigated.

\section{Results and discussion}

\subsection{A comparison study}

Consider doubly parallel current-carrying silver NWs with the following geometry and mechanical data: $r_{0}=5 \mathrm{~nm}, E_{b}=76 \mathrm{GPa}, \nu=0.26, \rho_{b}=10500 \mathrm{~kg} / \mathrm{m}^{3}, \rho_{0}=10^{-7} \mathrm{~kg} / \mathrm{m}^{2}$, $\lambda_{0}=1 \mathrm{~N} / \mathrm{m}, \mu_{0}=0.11 \mathrm{~N} / \mathrm{m}$, and $\tau_{0}=0.89 \mathrm{~N} / \mathrm{m}$. In order to ensure on the predicted frequencies via RKPM, such results should be checked with those of another methodology. To this end, assumed mode method (AMM) is implemented as an alternative approach. In AMM, the deformation fields are taken into account as a linear combination of admissible 
mode shapes. These modes should at least satisfy the essential boundary conditions of the problem. For simply supported DCCNWs, the $n$th mode shapes are considered as follows:

$$
\begin{aligned}
& \phi_{n}^{v}(\xi)=\phi_{n}^{w}(\xi)=\sin (n \pi \xi), \text { for RBT; } \\
& \phi_{n}^{v}(\xi)=\phi_{n}^{w}(\xi)=\sin (n \pi \xi), \phi_{n}^{\theta_{y}}(\xi)=\phi_{n}^{\theta_{z}}(\xi)=\cos (n \pi \xi), \text { for TBT; } \\
& \phi_{n}^{v}(\xi)=\phi_{n}^{w}(\xi)=\sin (n \pi \xi), \phi_{n}^{\psi_{y}}(\xi)=\phi_{n}^{\psi_{z}}(\xi)=\cos (n \pi \xi), \text { for HOBT. }
\end{aligned}
$$

By substituting the above-mentioned mode shapes into the mass and stiffness submatrices pertinent to the RBT, TBT, and HOBT as shape functions, their elements could be explicitly evaluated. Subsequently, the natural frequencies of the nanosystem would be readily obtainable using the suggested models. For the numerical analysis of each nanowire on the basis of the RKPM, 13 particles with uniform distribution, 12 computational cells, 4 Gaussian points in each cell, cubic spline window function, and linear base function are used. The dilation parameter is also set equal to 3.2 inter-particle distance. For frequency analysis of the problem using AMM, the first thirteen modes of vibration are considered. The predicted first, third, fifth, seventh, and ninth natural frequencies of the nanosystem via both AMM and RKPM based on the RBT, TBT, and HOBT are given in Table 1 . The results are provided for three levels of the slenderness ratio (i.e., $\lambda=10,20$, and 30) and two levels of the electric current (i.e., $\bar{I}_{0}^{*}=0.2$ and 0.5 ). In all cases, there is a reasonably good agreement between the predicted natural frequencies by the RKPM and those of the AMM. Generally, the natural frequencies would reduce as the electric current or slenderness ratio increases. Additionally, the discrepancies between the predicted results by various models would decrease by increasing of the slenderness ratio. In the upcoming parts, the more accurate roles of the influential parameters on the free dynamic response of the nanosystem will be displayed in some detail.

\subsection{A convergence check}

To show the efficiency of the suggested numerical models based on the RKPM, a convergence check is carried out. In Table 2, the predicted fundamental frequencies of the 
nanosystem (with unit $\mathrm{GHz}$ ) have been provided according to the exact version of magnetic forces for RBT, TBT, and HOBT. The results are given for four levels of the slenderness ratio (i.e., $\lambda=10,15,20$, and 40) and five numbers of RKPM's particles (i.e., $N P_{i}=5,7$, 13, 17, and 22) for particular values of the electric current, magnetic field strength, and interwire distance. According to Table 2, the predicted fundamental frequencies by various models would converge to specific values such that the discrepancies between the predicted results and these specific ones would decrease by increasing of the number of RKPM's particle. Such a fact holds true for all considered values of the slenderness ratio. Commonly, the predicted results by the TBT and HOBT are close to each other and the RBT overestimates the results of these models due to excluding the shear deformation effect in its formulations. In most of the cases, the results of the TBT and HOBT with $N P_{1}=N P_{2}=17$ capture those of $N P_{1}=N P_{2}=22$ with accuracy up to three significant digits. In the reminding of this paper, 13 RKPM's particles are exploited for discretization of the deformation fields associated with DCCNWs.

\subsection{Exact models vs. approximate models}

To investigate the accuracy of the proposed approximate models in predicting free vibration of magnetically affected DCCNWs, an interesting comparison study is performed. In Figs. 2(a)-2(c), the predicted fundamental frequencies by the RBT, TBT, and HOBT based on the approximate and exact versions of the magnetic force as a function of the interwire distance have been demonstrated. The results are provided for three levels of the

electric current (i.e., $\bar{I}_{0}=0.1,0.5$, and 1 ) whereas the magnetic field strength, the interwire distance, and the slenderness ratios of the CCNWs are kept fixed (i.e., $\bar{B}_{0}=50, d=5 r_{0}$, and $\lambda=10)$. The results of the exact models and the approximate models in order are plotted by the solid and dashed lines. Generally, the predicted fundamental frequencies by the exact models are underestimated by those of the approximate models. By increasing of the interwire distance, the predicted fundamental frequencies by all proposed models would in- 
crease. In fact, the influence of the Lorentz magnetic force of the neighboring CCNW on the dynamic deformation would reduce as the interwire distance grows. It implies that the lateral stiffness of the constitutive CCNWs would increase as the interwire distance increases. As a result, the natural frequencies would grow with the interwire distance. The rate of increase of fundamental frequencies in terms of interwire distance is more obvious in the approximate models, particularly for higher levels of the electric current. Additionally, the discrepancies between the results of the exact models and those of the approximate models would reduce as the interwire distance increases. It is mainly because of this fact that the effect of the dynamically exerted magnetic force would decay with the interwire distance. It can be imagined that for large enough interwire distance, the dynamic interactions between magnetically affected DCCNWs vanish and each CCNW vibrates individually, irrespective of the interwire distance. Commonly, the discrepancies between the predicted fundamental frequencies by the exact models and those of the approximate models would grow as the electric current increases. Such a fact holds true for all suggested models. For instance, the maximum relative discrepancies between the predicted results by the approximate models and those of the exact models on the basis of the HOBT would be about $0.0185,0.5$, and 2.9 percent for $\bar{I}_{0}=0.1,0.5$, and 1 , respectively.

\subsection{Numerical studies}

An inclusive parametric study is carried out to explain the roles of the influential factors on the free dynamic behavior of the nanosystem via RKPM. In this part, the effects of the electric current, magnetic field strength, slenderness ratio, and CCNW's radius on the fundamental frequencies are discussed under various physical conditions. The influences of the surface energy and the shear deformation effects on the obtained results are also studied. In all demonstrated results in this part, the plots associated with the surface elasticity theory of Gurtin-Murdoch and classical elasticity theory are specified by the solid and dashed lines, respectively. Furthermore, the plotted results with square, circle, and triangle markers in 
order present the obtained results based on the RBT, TBT, and HOBT.

\subsubsection{Effect of the electric current}

An important study is performed to determine the role of the electric current on the free transverse vibration of the nanosystem. In Figs. 3(a)-3(c), the plots of the predicted fundamental frequencies by the RBT, TBT, and HOBT as a function of a measuring parameter of the electric current are provided for three levels of the slenderness ratio (i.e., $\lambda=10$, 20, and 40) in the case of $d=5 r_{0}, \bar{B}_{0}^{*}=50$, and $\lambda^{*}=10$. The dimensionless electric current and the magnetic field strength (i.e., $\bar{I}_{0}$ and $\bar{B}_{0}$ ) are defined such that the variation of the slenderness ratio has no influence on the magnitudes of the electric current and magnetic field strength. The plotted results consist of two apparent parts: positive branches and negative branches. Actually, the positive branches show the natural frequencies in which the nanosystem could freely vibrate at them. The negative branches display the dynamically unstable parts in which under the given circumferences the nanosystem would be no longer stable. In such a case, the suggested models based on the small deformations predict that by enforcing a small initial deformation to the CCNWS, the displacements and stresses would grow as time passes until the nanosystem collapses. Therefore, identification of such critical zones is of great advantage for design purposes and practical applications. According to Figs. 3(a)-3(c), the natural frequencies would reduce as the electric current increases, irrespective of the slenderness ratio of the nanosystem. The rate of reduction is more obvious for higher levels of the slenderness ratio. It implies that the variation of the electric current is more influential on the variation of the frequencies of the magnetically affected DCCNWs with higher slenderness ratios. For particular levels of the electric current, the fundamental frequency of the nanosystem becomes zero and the nanosystem arrives at the dynamically unstable scenario. The electric current corresponds to zero fundamental frequencies is called critical electric current. As it is seen in Figs. 3(a)-3(c), the values of the critical electric currents would lessen as the slenderness ratio increases. Additionally, Fig. 3(c) displays 
that there exists two sets of critical electric currents. This matter is clarified with a more detail in the following. Based on the TBT and HOBT in the case of $\lambda=40$, the nanosystem will arrive at the dynamic instability as $\bar{I}_{0}^{*}$ becomes greater than 0.055 . However, if we enforce the nanosystem to mainly vibrate in the second modes (for example, by preventing the CCNWs from any lateral deformation at their midspan points), larger levels of the electric current would lead to initiation of the dynamic instability of the nanosystem. For the aforementioned case, the TBT and HOBT predict that the frequencies of the second modes become zero at $\bar{I}_{0}^{*} \approx 0.4$ and 0.41 , respectively.

A brief survey of the demonstrated results in Figs. 3(a)-3(c) reveals that the discrepancies between the results of the RBT and those of the HOBT would increase as the electric current increases. In most of the cases, the predicted fundamental frequencies by the RBT are greater than those obtained by the TBT and HOBT. Furthermore, the results of the TBT are closer to those of the HOBT since both models take into account the shear deformation effect as well as the rotational inertia of the CCNWs in their formulations. It implies that the predicted transverse stiffness by the TBT and HOBT is lower than that predicted by the RBT since the later model does not consider the shear effect.

\subsubsection{Effect of the magnetic field strength}

Another crucial scrutiny is carried out to display the role of the strength of the applied magnetic field on the free vibration of the magnetically affected DCCNWs on the basis of the proposed models using the exact version of the exerted magnetic forces. The predicted frequencies as a function of the magnetic field strength are plotted in Figs. 4(a)-4(c) for different levels of the slenderness ratio. The results have been provided for a nanosystem with $\bar{I}_{0}^{*}=0.3, d=5 r_{0}$, and $\lambda^{*}=10$ such that the dimensionless values of the electric current and magnetic field strength are calculated by: $\bar{I}_{0}=\bar{I}_{0}^{*}\left(\frac{\lambda}{\lambda^{*}}\right)$ and $\bar{B}_{0}=\bar{B}_{0}^{*}\left(\frac{\lambda}{\lambda^{*}}\right)^{2}$. According to the plotted results, the predicted fundamental frequencies by various beam models would reduce by increasing of the magnetic field strength. In fact, the lateral stiffness of the 
CCNWs would reduce as the strength of the applied magnetic field grows. The rate of reduction of the frequencies in terms of the magnetic field strength is more apparent for higher values of the magnetic fields. The corresponding magnetic field strength of the zero frequencies is called critical magnetic field strength. It is worth mentioning that the magnitudes of such critical values for the nanosystem at hand depends on the electric current, length and radius of the CCNWs, and the interwire distance. Herein, only the influence of the slenderness ratio on the critical magnetic field strength is addressed based on the suggested models. Figs. 4(a)-4(c) display that the critical magnetic field strength would reduce as the slenderness ratio of the constitutive NWs of the nanosystem increases. In other words, for nanosystems under identical physical conditions, the influence of the magnetic field strength on the natural frequencies of the nanosystem with higher slenderness ration is more obvious. For example, the nanosystem with $\lambda=10$ is almost stable for the applied magnetic fields of strength in the range $10 \leq \bar{B}_{0}^{*} \leq 100$. In the case of $\lambda=20$, the nanosystem becomes unstable at $\bar{B}_{0}^{*} \approx 55$ based on the TBT and HOBT; however, the RBT predicts that the nanosystem is dynamically unstable at $\bar{B}_{0}^{*} \approx 65$. Finally, in the case of $\lambda=40$, the RBT, TBT, and HOBT predict that the nanosystem will arrive at the unstable zone of the first vibration mode as the magnetic field strength passes $\bar{B}_{0}^{*} \approx 10.5,11$, and 11.5 , respectively.

Irrespective of the slenderness ratio, the relative discrepancies between the predicted fundamental frequencies by the RBT or TBT and those of the HOBT would commonly magnify as the applied magnetic field becomes stronger. The maximum relative discrepancies are observed approximately near the critical magnetic field strength. Generally, the predicted results by the TBT and HOBT are overestimated by the RBT. Concerning the surface effect, the predicted fundamental frequencies without considering the surface energy are generally lower than those obtained by consideration of the surface effect. Additionally, the relative discrepancies between the obtained results by considering the surface effect and those obtained without considering the surface energy would grow with the magnetic field strength and reach to their maximum values at the critical magnetic field strength. 


\subsubsection{Effect of the slenderness ratio}

Equally important is to explore the role of the slenderness ratio on the free transverse vibrations of the magnetically affected DCCNWs. The plots of the predicted fundamental frequencies by various models as a function of the slenderness ratio are now demonstrated in Figs. 5(a)-5(c). The results are provided for three levels of the electric current (i.e., $\bar{I}_{0}^{*}=0.5$, 1 , and 2) in the case of $\bar{B}_{0}^{*}=50$ and $d=5 r_{0}$. To neutralize the influence of the slenderness ratio on the dimensionless values of both electric current and magnetic field strength, their dimensionless values are defined by: $\bar{I}_{0}=\bar{I}_{0}^{*}\left(\frac{\lambda}{\lambda^{*}}\right)$ and $\bar{B}_{0}=\bar{B}_{0}^{*}\left(\frac{\lambda}{\lambda^{*}}\right)^{2}$. According to the demonstrated results in Figs. 5(a)-5(c), the fundamental frequency of the magnetically affected nanosystem decreases as the slenderness ratio grows. For particular levels of the slenderness ratio, the so-called critical slenderness ratio, the predicted fundamental frequencies by the proposed models would become zero. Actually, for a nanosystem with a slenderness ratio greater than that of the critical one, the nanosystem is inherently unstable. If an initial deformation is enforced to such a nanosystem and allow that to vibrate freely, the amplitudes of dynamic displacement response would drastically grow as the time goes by and the nanosystem will collapse sooner or later. The electric current, magnetic field strength, interwire distance, surface energy, and shear deformation are among the major factors that influence on the critical slenderness ratios. Based on Figs. 5(a)-5(c), only the roles of the surface energy effect and the electric current on the critical slenderness ratios could be explained. As it is obvious, the critical slenderness ratio would reduce as the electric current through the nanowires grows. In other words, the nanosystem carries a higher electric current becomes dynamically unstable at lower levels of the slenderness ratio. Such a fact holds true for all suggested models. For the considered nanosystem, the surface energy helps the stability and transverse stiffness of the nanosystem. As a result, the predicted critical slenderness ratios by the proposed models accounting for the surface energy are generally greater than those without consideration of the surface effect. As explained, it is mainly related to this fact that the Young's modulus of the surface layer of the silver NWs 
has a positive value. Therefore, considering the surface layer leads to a stiffer nanosystem in compare to the case that only bulk is taken into account.

Regarding the surface energy effect, the relative discrepancies between the predicted fundamental frequencies by considering the surface energy and those obtained without consideration of the surface effect would commonly increase as the slenderness ratio grows. Such a fact is more obvious for those nanosystems carry larger amount of electric currents. In fact, the role of the surface energy effect becomes more important as the intensity of the electric current increases. Furthermore, the maximum of the above-mentioned discrepancies occurs at the critical slenderness ratio for each model. Concerning the capabilities of the RBT and the TBT in capturing the predicted fundamental frequencies by the HOBT, a close scrutiny of the plotted results indicates that the relative discrepancies between the predicted results by the RBT or TBT and those of the HOBT would reduce with the slenderness ratio up to a particular value. For slenderness ratio greater than this particular value, such relative discrepancies would grow with the slenderness ratio up to its critical value. It implies that the shear deformation becomes very important when examining free dynamic response of the magnetically affected nanosystem with slenderness ratios close to the critical value is of concern.

\subsubsection{Effect of the CCNW's radius}

The role of the radius of the CCNWs in free vibration of the magnetically affected nanosystem is also of high interest. For this purpose, the plots of the fundamental frequencies as a function of the radius of CCNWs for three levels of the electric current (i.e., $\bar{I}_{0}^{*}=0.5,1$, and 2) are provided in Figs. 6(a)-6(c). The plotted results are given for a DCCNWs with $\lambda=15$ and $d=5 r_{0}$ in which subjected to a longitudinal magnetic field of dimensionless strength $\bar{B}_{0}=\bar{B}_{0}^{*}\left(\frac{r_{0}^{*}}{r_{0}}\right)^{2}$ where $\bar{B}_{0}^{*}=50$. Additionally, the dimensionless value of the electric current within the CCNWs is taken into account as: $\bar{I}_{0}=\bar{I}_{0}^{*}\left(\frac{r_{0}^{*}}{r_{0}}\right)^{2}$. The present dimensionless versions of the magnetic field strength and electric current have been considered such that 
the variation of the radius of the CCNWs would have no influence on the variation of the magnetic field as well as the electric current intensity. As it is seen in Figs. 6(a)-6(c), the fundamental frequency of the nanosystem would grow as the radius of the CCNWs increases. It is mainly because of this fact that the increasing of the transverse stiffness of the nanosystem occurs with a higher rate in compare to the increasing of its mass as the radius grows. Additionally, variation of the CCNWs' radius is more influential on the variation of the fundamental frequencies of the nanosystem with lower radius passes higher electric currents.

Regarding the discrepancies between the obtained results by the proposed numerical models, the TBT could capture the predicted results by the HOBT for $\bar{I}_{0}^{*}=0.5,1$, and 2 with relative error lower than $1.7,1.8$, and 4.6 percent, respectively. In the case of $\bar{I}_{0}^{*}=0.5$, the relative discrepancies between the results of the RBT and those of the HOBT would commonly magnify as the radius of the CCNWs increases. In the cases of $\bar{I}_{0}^{*}=1$ and 2 , the above-mentioned relative discrepancies would reduce as the radius of the CCNWs increases up to a particular value. For radii greater than such a particular value, the discrepancies between the predicted results by the RBT and those of the HOBT would commonly magnify as the radius of the CCNWs grows. In view of this fact, for the considered range of the CCNWs' radius, the RBT overestimates the predicted fundamental frequencies by the HOBT with relative error lower than 11.5, 11.6, and 21.8 percent. Concerning the surface effect, the relative discrepancies between the obtained results by considering the surface energy effect and those obtained without considering the surface effect, would commonly decrease as the radius of the CCNWs increases. Furthermore, such discrepancies magnify as the electric current intensity increases. It indicates that the surface energy effect becomes more crucial in magnetically affected nanosystems carry higher levels of the electric currents. 


\section{Conclusions}

Free transverse vibration of magnetically affected DCCNWs is of high concern due to the potential applications of vertically aligned CCNWs in nanogenerators, nanosensors, and NEMS. As a result, the problem and their dynamic interactions are carefully explored by developing approximate and exact models for evaluating the mutually exerted magnetic forces on the CCNWs. In the context of the Gurtin-Murdoch elasticity theory, the equations of motion of the nanosystem are constructed using the RBT, TBT, and HOBT. In accordance with the approximate models, these are in order four, eight, and eight coupled PDEs while the exact model displays that these are four, eight, and eight coupled IPDEs, respectively. By exploiting RKPM, the frequencies of the magnetically affected nanosystem are evaluated and the obtained results are also successfully checked with those of the AMM. The roles of the electric current intensity, magnetic field strength, interwire distance, and radius of the CCNWs on the predicted fundamental frequencies of the nanosystem based on the suggested exact models are also investigated. For each parametric study, the effects of both surface energy and shear deformation on the obtained results are explained and discussed.

The proposed models and the obtained results can be regarded as a solid basis for better understanding the mechanical response of more complex nanosystems consist of vertically aligned nanowires carrying electric currents. The free and forced dynamic behaviors of the later nanosystem in a magnetic field are also of high interest. These subjects display the hot topics in the unknown realms of the applied mechanics as well as nanotechnology. More explorations on these themes would lead to the optimal and rational design of groups of CCNWs which are anticipated to be building blocks of the upcoming advanced nanodevices. 


\section{Acknowledgement}

The financial supports of Iran National Science Foundation (INSF), research department of K.N. Toosi University of Technology, and Iran Nanotechnology Initiative Council is gratefully acknowledged. 


\section{References}

Ando, B., Baglio, S., Bulsara, A. R., \& Trigona, C. (2009). Design and characterization of a microwire fluxgate magnetometer. Sensors and Actuators A: Physical, 151, 145-153.

Ansari, R., Mohammadi, V., Shojaei, M. F., \& Sahmani, S. (2014). On the forced vibration analysis of Timoshenko nanobeams based on the surface stress elasticity theory. Composites Part B: Engineering, 60, 158-166.

Ansari, R., Mohammadi, V., Faghih Shojaei, M., Gholami, R., \& Sahmani, S. (2014). Postbuckling analysis of Timoshenko nanobeams including surface stress effect. International Journal of Engineering Science, 75, 1-10.

Ansari, R., \& Sahmani, S. (2011). Surface stress effects on the free vibration behavior of nanoplates. International Journal of Engineering Science, 49, 1204-1215.

Assadi, A. (2013). Size dependent forced vibration of nanoplates with consideration of surface effects. Applied Mathematical Modeling, 37, 3575-3588.

Bickford, W. B. (1982). A consistent higher order beam theory. Developments in Theoretical and Applied Mechanics, 11, 137-150.

Chen, J. S., Roque, C. M. O. L., Pan, C., \& Button, S. T. (1998). Analysis of metal forming process based on meshless method. Journal of Materials Processing Technology, 80, 642646.

Cheng, R., \& Liew, K. M. (2009). The reproducing kernel particle method for twodimensional unsteady heat conduction problems. Computational Mechanics, 45, 1-10.

Cui, Y., \& Lieber, C. M. (2001). Functional nanoscale electronic devices assembled using silicon nanowire building blocks. Science, 291, 851-853.

Dingreville, R., Qu, J., \& Cherkaoui, M. (2005). Surface free energy and its effect on the elastic behavior of nano-sized particles, wires and films. Journal of the Mechanics and Physics of Solids, 53, 1827-1854.

Duan, X., Huang, Y., Cui, Y., Wang, J., \& Lieber, C. M. (2001). Indium phosphide nanowires as building blocks for nanoscale electronic and optoelectronic devices. Nature, 
409, 66-69.

Eltaher, M. A., Emam, S. A., \& Mahmoud, F. F. (2012). Free vibration analysis of functionally graded size-dependent nanobeams. Applied Mathematics and Computation, 218, 7406-7420.

Farajpour, A., Dehghany, M., \& Shahidi, A. R. (2013). Surface and nonlocal effects on the axisymmetric buckling of circular graphene sheets in thermal environment. Composites Part B: Engineering, 50, 333-343.

Gheshlaghi, B., \& Hasheminejad, S. M. (2011). Surface effects on nonlinear free vibration of nanobeams. Composites Part B: Engineering, 42, 934-937.

Gheshlaghi, B., \& Hasheminejad, S. M. (2012). Vibration analysis of piezoelectric nanowires with surface and small scale effects. Current Applied Physics, 12, 1096-1099.

Gurtin, M. E., \& Murdoch, A. I. (1975). A continuum theory of elastic material surfaces. Archive for Rational Mechanics and Analysis, 57, 291-323.

Gurtin, M. E., \& Murdoch, A. I. (1976). Effect of surface stress on wave propagation in solids. Journal of Applied Physics, 47, 4414-4421.

Gurtin, M. E., \& Murdoch, A. I. (1978). Surface stress in solids. International Journal of Solids and Structures, 14, 431-440.

Hahm, J. I., \& Lieber, C. M. (2004). Direct ultrasensitive electrical detection of DNA and DNA sequence variations using nanowire nanosensors. Nano Letters, 4, 51-54.

Hashemi, S.H., \& Nazemnezhad, R. (2013). An analytical study on the nonlinear free vibration of functionally graded nanobeams incorporating surface effects. Composites Part B: Engineering, 52, 199-206.

Huang, D. W. (2008). Size-dependent response of ultra-thin films with surface effects. International Journal Solids and Structures, 45, 568-579.

Ji-fa, Z., Wen-Pu, Z., \& Yao, Z. (2005). A meshfree method and its applications to elastoplastic problems. Journal of Zhejiang University Science A, 6, 148-154.

Jing, G. Y., Duan, H., Sun, X. M., Zhang, Z. S., Xu, J., Li, Y. D., ... \& Yu, D. P. (2006). 
Surface effects on elastic properties of silver nanowires: contact atomic-force microscopy. Physical Review B, 73, 235409.

Khajeansari, A., Baradaran, G. H., \& Yvonnet, J. (2012). An explicit solution for bending of nanowires lying on Winkler-Pasternak elastic substrate medium based on the EulerBernoulli beam theory. International Journal of Engineering Science, 52, 115-128.

Kiani, K. (2010). A meshless approach for free transverse vibration of embedded single-walled nanotubes with arbitrary boundary conditions accounting for nonlocal effect. International Journal of Mechanical Sciences, 52, 1343-1356.

Kiani, K. (2014). Surface effect on free transverse vibrations and dynamic instability of current-carrying nanowires in the presence of a longitudinal magnetic field. Physics Letters A, 378, 1834-1840.

Kiani, K. (2014). Forced vibrations of a current-carrying nanowire in a longitudinal magnetic field accounting for both surface energy and size effects. Physica E: Low-dimensional Systems and Nanostructures, 63, 27-35.

Kiani, K. (2015). Axial buckling analysis of a slender current-carrying nanowire acted upon by a magnetic field using the surface energy approach. Journal of Physics D: Applied Physics, 48, 245302.

Kiani, K. (2015). Column buckling of magnetically affected stocky nanowires carrying electric current. Journal of Physics and Chemistry of Solids, 83, 140-151.

Kiani, K. (2015). Stability and vibrations of doubly parallel current-carrying nanowires immersed in a longitudinal magnetic field. Physics Letters A, 379, 348-360.

Kiani, K. (2015). Vibrations and instability of double-nanowire-systems as electric current carriers. Modern Physics Letters B, 29, 1550144.

Li, Y., Song, J., Fang, B., \& Zhang, J. (2011). Surface effects on the postbuckling of nanowires. Journal of Physics D: Applied Physics, 44, 425304.

Liew, K. M., Ng, T. Y., \& Wu, Y. C. (2002). Meshfree method for large deformation analysisa reproducing kernel particle approach. Engineering Structures, 24, 543-551. 
Liu, W. K., Jun, S., \& Zhang, Y. F. (1995). Reproducing kernel particle methods. International Journal for Numerical Methods in Fluids, 20, 1081-1106.

Liu, J. L., Mei, Y., Xia, R., \& Zhu, W. L. (2012). Large displacement of a static bending nanowire with surface effects. Physica E: Low-dimensional Systems and Nanostructures, 44, 2050-2055.

Liu, W. K., Jun, S., Li, S., Adee, J., \& Belytschko, T. (1995). Reproducing kernel particle methods for structural dynamics. International Journal for Numerical Methods in Engineering, 38, 1655-1679.

Liu, W. K., Jun, S., Sihling, D. T., \& Hao, W. (1997). Multiresolution reproducing kernel particle method for computational fluid dynamics. International Journal for Numerical Methods in Fluids, 24, 1391-1415.

Lu, T. Q., Zhang, W. X., \& Wang, T. J. (2011). The surface effect on the strain energy release rate of buckling delamination in thin filmsubstrate systems. International Journal of Engineering Science, 49, 967-975.

Malekzadeh, P., Haghighi, M. G., \& Shojaee, M. (2014). Nonlinear free vibration of skew nanoplates with surface and small scale effects. Thin Walled Structures, 78, 48-56.

Malekzadeh, P., \& Shojaee, M. (2013). Surface and nonlocal effects on the nonlinear free vibration of non-uniform nanobeams. Composites Part B: Engineering, 52, 84-92.

Miller, R. E., \& Shenoy, V. B. (2000). Size-dependent elastic properties of nanosized structural elements. Nanotechnology, 11, 13.

Nianfei, G., Guangyao, L., \& Shuyao, L. (2009). 3D adaptive RKPM method for contact problems with elastic-plastic dynamic large deformation. Engineering Analysis with Boundary Elements, 33, 1211-1222.

Olsson, P. A. T., \& Park, H. S. (2012). On the importance of surface elastic contributions to the flexural rigidity of nanowires. Journal of the Mechanics and Physics of Solids, 60, 2064-2083.

Park, H. S. (2012). Surface stress effects on the critical buckling strains of silicon nanowires. 
Computational Materials Science, 51, 396-401.

Park, H. S., \& Klein, P. A. (2008). Surface stress effects on the resonant properties of metal nanowires: The importance of finite deformation kinematics and the impact of the residual surface stress. Journal of the Mechanics and Physics of Solids, 56, 3144-3166.

Pishkenari, H. N., Afsharmanesh, B., \& Tajaddodianfar, F. (2016). Continuum models calibrated with atomistic simulations for the transverse vibrations of silicon nanowires. International Journal of Engineering Science, 100, 8-24.

Qin, Y., Wang, X., \& Wang, Z. L. (2008). Microfibrenanowire hybrid structure for energy scavenging. Nature, 451, 809-813.

Ravari, M. K., \& Shahidi, A. R. (2013). Axisymmetric buckling of the circular annular nanoplates using finite difference method. Meccanica, 48, 135-144.

Reddy, J. N. (1984). A simple higher-order theory for laminated composite plates. Journal of Applied Mechanics, 51, 745-752.

Sahmani, S., Bahrami, M., \& Aghdam, M.M. (2016). Surface stress effects on the nonlinear postbuckling characteristics of geometrically imperfect cylindrical nanoshells subjected to axial compression. International Journal of Engineering Science, 99, 92-106.

Timoshenko, S. P. (1921). On the correction for shear of the differential equation for transverse vibrations of prismatic bars. The London, Edinburgh, and Dublin Philosophical Magazine and Journal of Science, 41, 744-6.

Timoshenko, S. P. (1922). On the transverse vibrations of bars of uniform cross-section. The London, Edinburgh, and Dublin Philosophical Magazine and Journal of Science, 43, $125-31$

Wagner, G. J., \& Liu, W. K. (2000). Application of essential boundary conditions in meshfree methods: a corrected collocation method. International Journal for Numerical Methods in Engineering, 47, 1367-1379.

Wang, G. F., \& Feng, X. Q. (2010). Effect of surface stresses on the vibration and buckling of piezoelectric nanowires. Europhysics Letters, 91, 56007. 
Wang, G. F., \& Feng, X. Q. (2009). Surface effects on buckling of nanowires under uniaxial compression. Applied Physics Letters, 94, 141913.

Wang, Z. L., \& Song, J. (2006). Piezoelectric nanogenerators based on zinc oxide nanowire arrays. Science, 312, 242-246.

Wang, X., Song, J., Liu, J., \& Wang, Z. L. (2007). Direct-current nanogenerator driven by ultrasonic waves. Science, 316, 102-105.

Wang, K. F., \& Wang, B. L. (2011). Vibration of nanoscale plates with surface energy via nonlocal elasticity. Physica E: Low-dimensional Systems and Nanostructures, 44, 448-453.

Wang, K. F., \& Wang, B. L. (2011). Combining effects of surface energy and non-local elasticity on the buckling of nanoplates. Micro and Nano Letters, 6, 941-943.

Wickenden, D. K., Champion, J. L., Osiander, R., Givens, R. B., Lamb, J. L., Miragliotta, J. A., ... \& Kistenmacher, T. J. (2003). Micromachined polysilicon resonating xylophone bar magnetometer. Acta Astronautica, 52, 421-425.

Wickenden, D. K., Kistenmacher, T. J., Osiander, R., Ecelberger, S. A., Givens, R. B., \& Murphy, J. C. (1997). Development of miniature magnetometers. Johns Hopkins APL Technical Digest, 18, 271-278.

Yan, Z., \& Jiang, L. Y. (2011). The vibrational and buckling behaviors of piezoelectric nanobeams with surface effects. Nanotechnology, 22, 245703.

Yan, Z., \& Jiang, L. Y. (2012). Vibration and buckling analysis of a piezoelectric nanoplate considering surface effects and in-plane constraints. Proceedings of the Royal Society A: Mathematical, Physical and Engineering Sciences, rspa20120214.

Zhang, L. T., Wagner, G. J., \& Liu, W. K. (2003). Modelling and simulation of fluid structure interaction by meshfree and FEM. Communications in Numerical Methods in Engineering, $19,615-621$.

Zhang, J., Wang, C., \& Adhikari, S. (2012). Surface effect on the buckling of piezoelectric nanofilms. Journal of Physics D: Applied Physics, 45, 285301. 


\section{Captions of the Tables:}

Table 1. Comparison between the natural frequencies of the nanosystem obtained by the RKPM and those of the AMM for different levels of the slenderness ratio and electric current $\left(\lambda^{*}=10, \bar{B}_{0}^{*}=10 ; d=5 r_{0} ; \bar{I}_{0}=\bar{I}_{0}^{*}\left(\frac{\lambda}{\lambda^{*}}\right), \bar{B}_{0}=\bar{B}_{0}^{*}\left(\frac{\lambda}{\lambda^{*}}\right)^{2} ; I_{0}=\bar{I}_{0} \sqrt{\frac{2 \pi E_{b} I_{b}}{\mu_{0} l_{b}^{2}}}, B_{0}=\right.$ $\left.\frac{\bar{B}_{0}}{l_{b}^{2}} \sqrt{\frac{\mu_{0} E_{b} I_{b}}{2 \pi}}\right)$

Table 2. Convergence check of the proposed meshless models for different values of the slenderness ratios $\left(\lambda^{*}=60, \bar{I}_{0}^{*}=0.4, \bar{B}_{0}^{*}=50, d=5 r_{0} ; \bar{I}_{0}=\bar{I}_{0}^{*}\left(\frac{\lambda}{\lambda^{*}}\right), \bar{B}_{0}=\bar{B}_{0}^{*}\left(\frac{\lambda}{\lambda^{*}}\right)^{2}\right)$. 


\section{Captions of the figures:}

Fig. 1. A current-carrying double-nanowire-system immersed in a longitudinal magnetic field.

Fig. 2. Variation of the fundamental frequency of magnetically affected DCCNWs in terms of interwire distance for different values of the electric current: (a) $\bar{I}_{0}=0.1$, (b) $\bar{I}_{0}=0.5$, (c) $\bar{I}_{0}=1 ;\left(\bar{B}_{0}=50, \lambda=10, \bar{f}_{0}^{E}=\bar{B}_{0} \bar{I}_{0}, \bar{f}_{1}^{E}=\left(\frac{\bar{I}_{0}}{\bar{d}}\right)^{2} ;(---)\right.$ approximate model, $(-)$ exact model; ( $\square)$ RBT, (०) TBT, $(\triangle)$ HOBT).

Fig. 3. Variation of the fundamental frequency of magnetically affected DCCNWs in terms of electric current for different levels of the slenderness ratio: (a) $\lambda=10$, (b) $\lambda=20$, (c) $\lambda=40 ;\left(\bar{B}_{0}^{*}=50, d=5 r_{0}, \lambda^{*}=10 ; \bar{I}_{0}=\bar{I}_{0}^{*}\left(\frac{\lambda}{\lambda^{*}}\right), \bar{B}_{0}=\bar{B}_{0}^{*}\left(\frac{\lambda}{\lambda^{*}}\right)^{2} ;(---)\right.$ without considering the surface effect, $(-)$ by considering the surface effect; ( $\square) \operatorname{RBT},(\circ) \mathrm{TBT},(\triangle) \mathrm{HOBT}$ ).

Fig. 4. Variation of the fundamental frequency of magnetically affected DCCNWs in terms of magnetic field strength for different levels of the slenderness ratio: (a) $\lambda=10$, (b) $\lambda=20,(\mathrm{c}) \lambda=40 ;\left(\bar{I}_{0}^{*}=0.3, d=5 r_{0}, \lambda^{*}=10 ; \bar{I}_{0}=\bar{I}_{0}^{*}\left(\frac{\lambda}{\lambda^{*}}\right), \bar{B}_{0}=\bar{B}_{0}^{*}\left(\frac{\lambda}{\lambda^{*}}\right)^{2} ;(---)\right.$ without considering the surface effect, (-) by considering the surface effect; ( $\square$ ) RBT, (o) TBT, $(\triangle) \mathrm{HOBT})$.

Fig. 5. Variation of the fundamental frequency of magnetically affected DCCNWs in terms of slenderness ratio for different levels of the electric current: (a) $\bar{I}_{0}^{*}=0.5$, (b) $\bar{I}_{0}^{*}=1$, (c) $\bar{I}_{0}^{*}=2 ;\left(\bar{B}_{0}^{*}=50, d=5 r_{0}, \lambda^{*}=10 ; \bar{I}_{0}=\bar{I}_{0}^{*}\left(\frac{\lambda}{\lambda^{*}}\right), \bar{B}_{0}=\bar{B}_{0}^{*}\left(\frac{\lambda}{\lambda^{*}}\right)^{2} ;(---)\right.$ without considering the surface effect, $(-)$ by considering the surface effect; ( $\square) \operatorname{RBT},(\circ) \mathrm{TBT},(\triangle) \mathrm{HOBT}$ ).

Fig. 6. Variation of the fundamental frequency of magnetically affected DCCNWs in terms of NW's radius for different levels of the electric current: (a) $\bar{I}_{0}^{*}=0.5$, (b) $\bar{I}_{0}^{*}=1$, (c) $\bar{I}_{0}^{*}=2 ;\left(r_{0}^{*}=5 \mathrm{~nm}, \bar{B}_{0}^{*}=50, d=5 r_{0}^{*}, \lambda=15 ; \bar{I}_{0}=\bar{I}_{0}^{*}\left(\frac{r_{0}^{*}}{r_{0}}\right)^{2}, \bar{B}_{0}=\bar{B}_{0}^{*}\left(\frac{r_{0}^{*}}{r_{0}}\right)^{2} ;(---)\right.$ without considering the surface effect, (-) by considering the surface effect; ( $\square$ ) RBT, (०) TBT, $(\triangle) \mathrm{HOBT})$. 


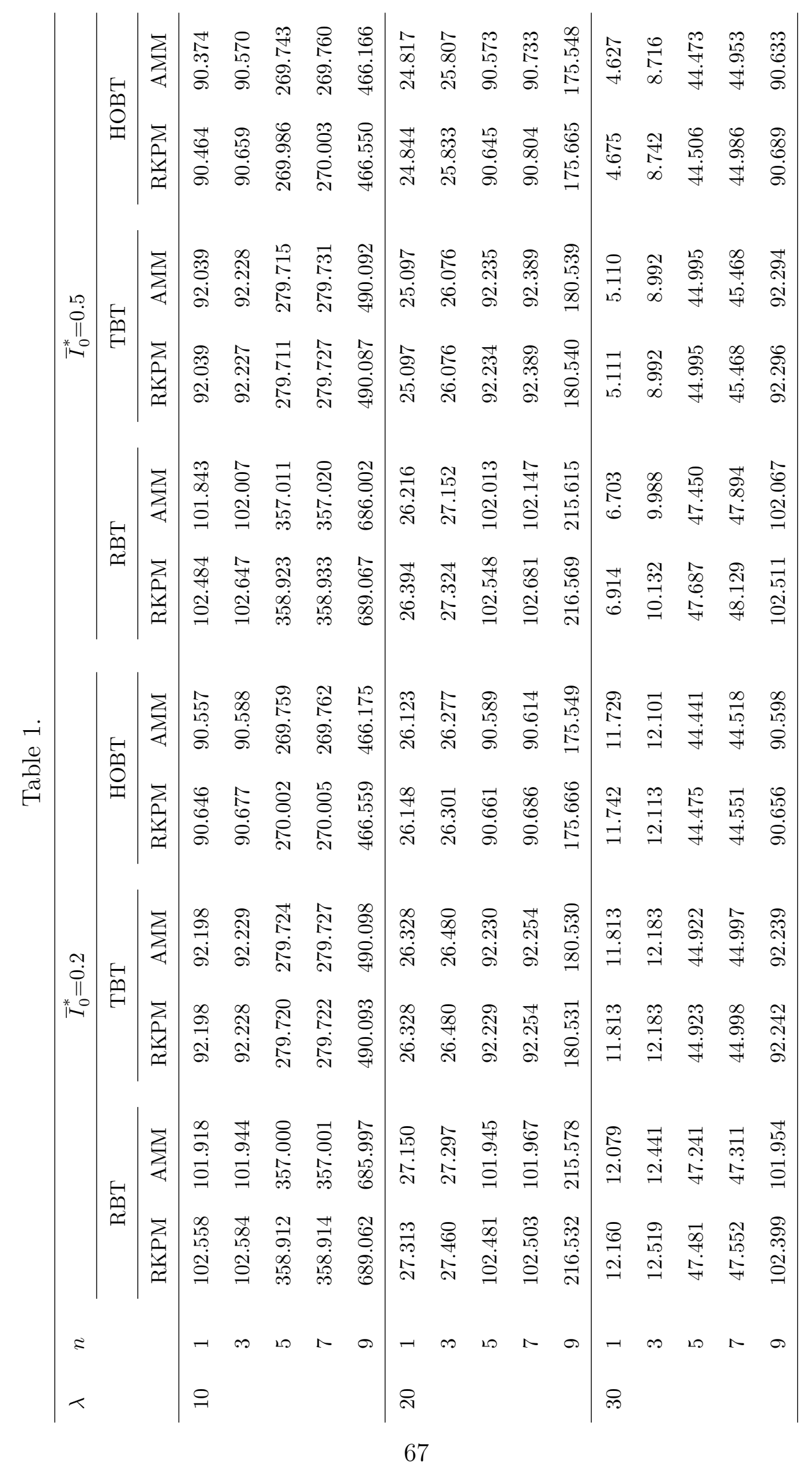


Table 2.

\begin{tabular}{ccccccc}
\hline$\lambda$ & Model & \multicolumn{5}{c}{$N P_{i}$} \\
\cline { 3 - 7 } & & 5 & 7 & 13 & 17 & 22 \\
\hline 10 & RBT & 102.1171 & 102.3326 & 102.5718 & 102.5982 & 102.6097 \\
& TBT & 92.2623 & 92.2361 & 92.2277 & 92.2276 & 92.2276 \\
& HOBT & 90.6163 & 90.6484 & 90.6800 & 90.6840 & 90.6858 \\
\hline \multirow{2}{*}{15} & RBT & 47.2786 & 47.3752 & 47.4829 & 47.4948 & 47.4999 \\
& TBT & 44.9230 & 44.9058 & 44.9010 & 44.9009 & 44.9008 \\
& HOBT & 44.4436 & 44.4556 & 44.4697 & 44.4715 & 44.4723 \\
\hline \multirow{2}{*}{20} & RBT & 27.3694 & 27.4230 & 27.4830 & 27.4897 & 27.4926 \\
& TBT & 26.5703 & 26.5571 & 26.5539 & 26.5538 & 26.5537 \\
& HOBT & 26.3745 & 26.3792 & 26.3869 & 26.3879 & 26.3884 \\
\hline & RBT & 7.7346 & 7.7461 & 7.7596 & 7.7611 & 7.7618 \\
& TBT & 7.6870 & 7.6784 & 7.6771 & 7.6769 & 7.6769 \\
& HOBT & 7.6570 & 7.6547 & 7.6561 & 7.6563 & 7.6564 \\
\hline
\end{tabular}




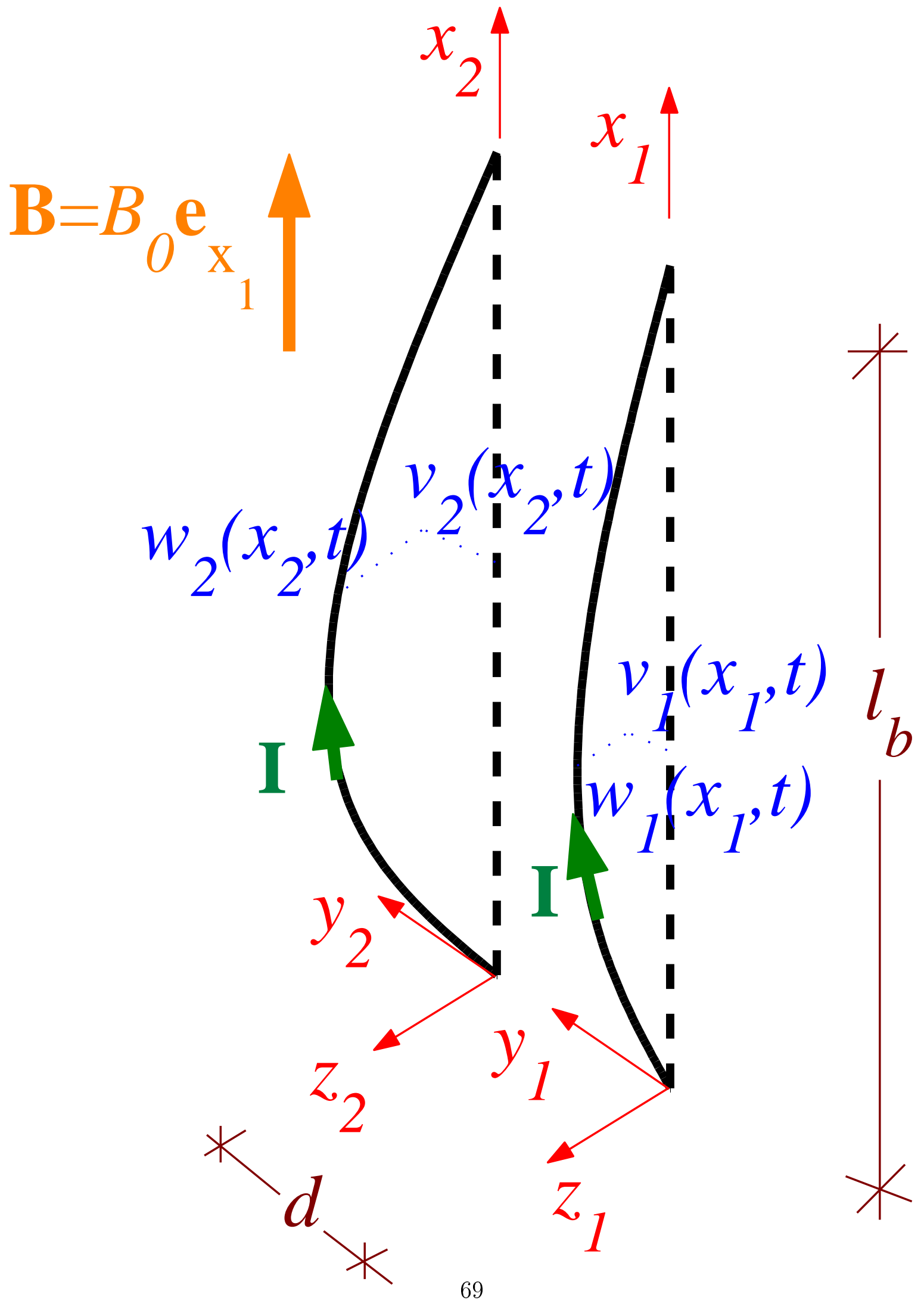

Fig. 1. 
(a)

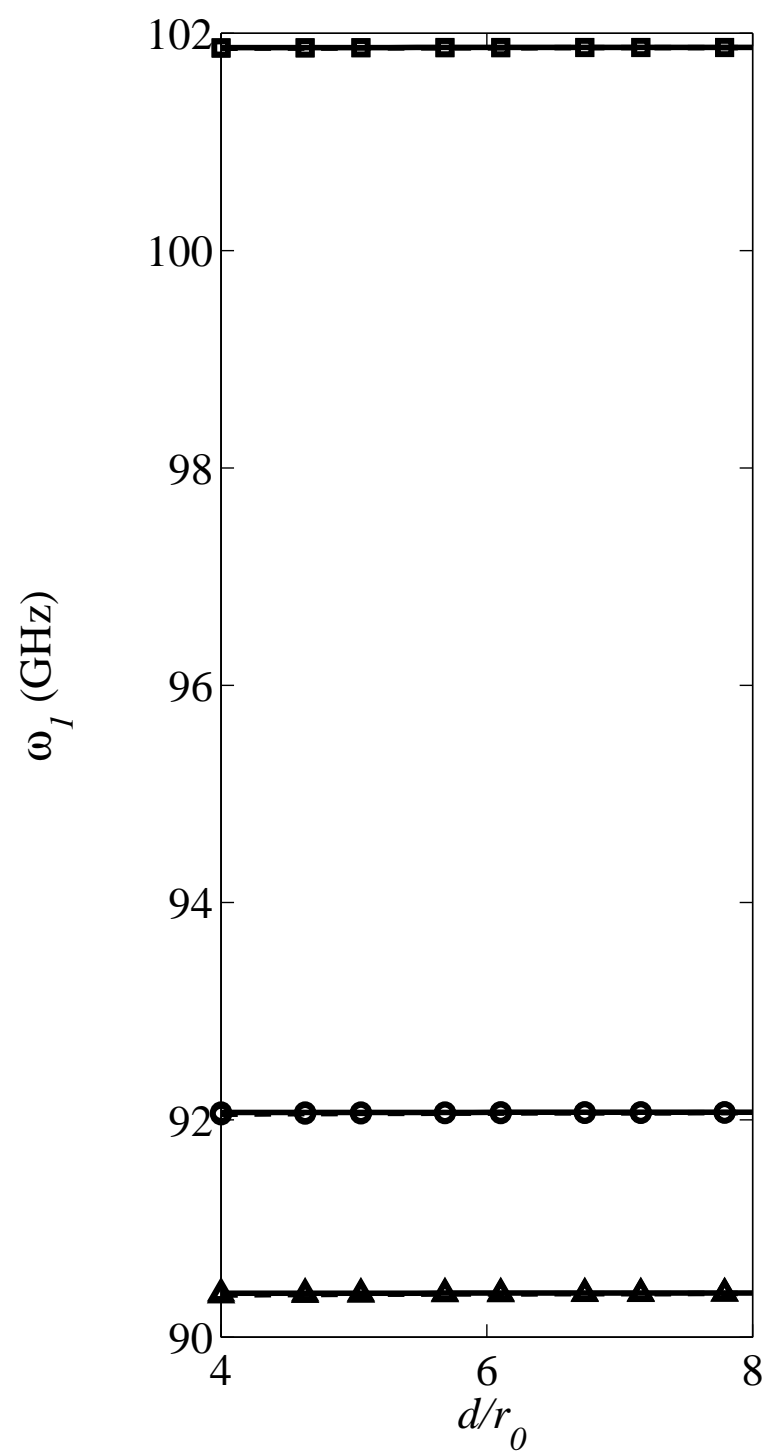

(b)

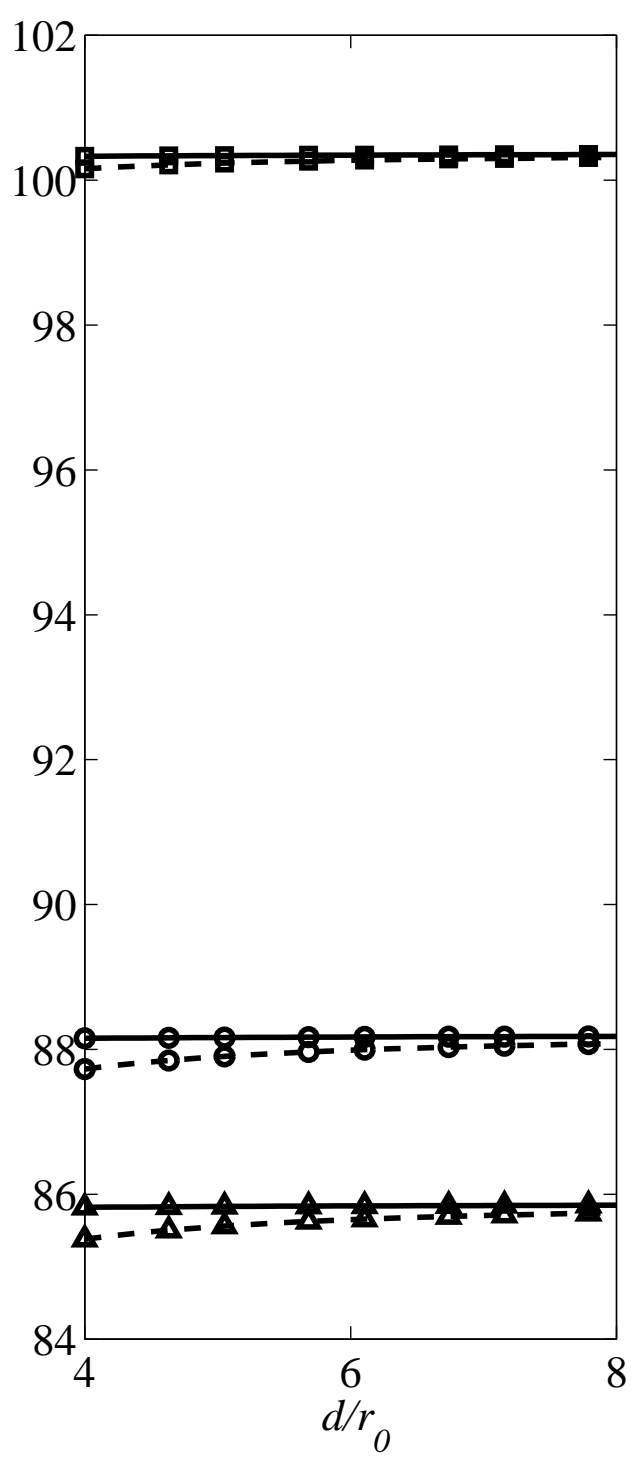

(c)

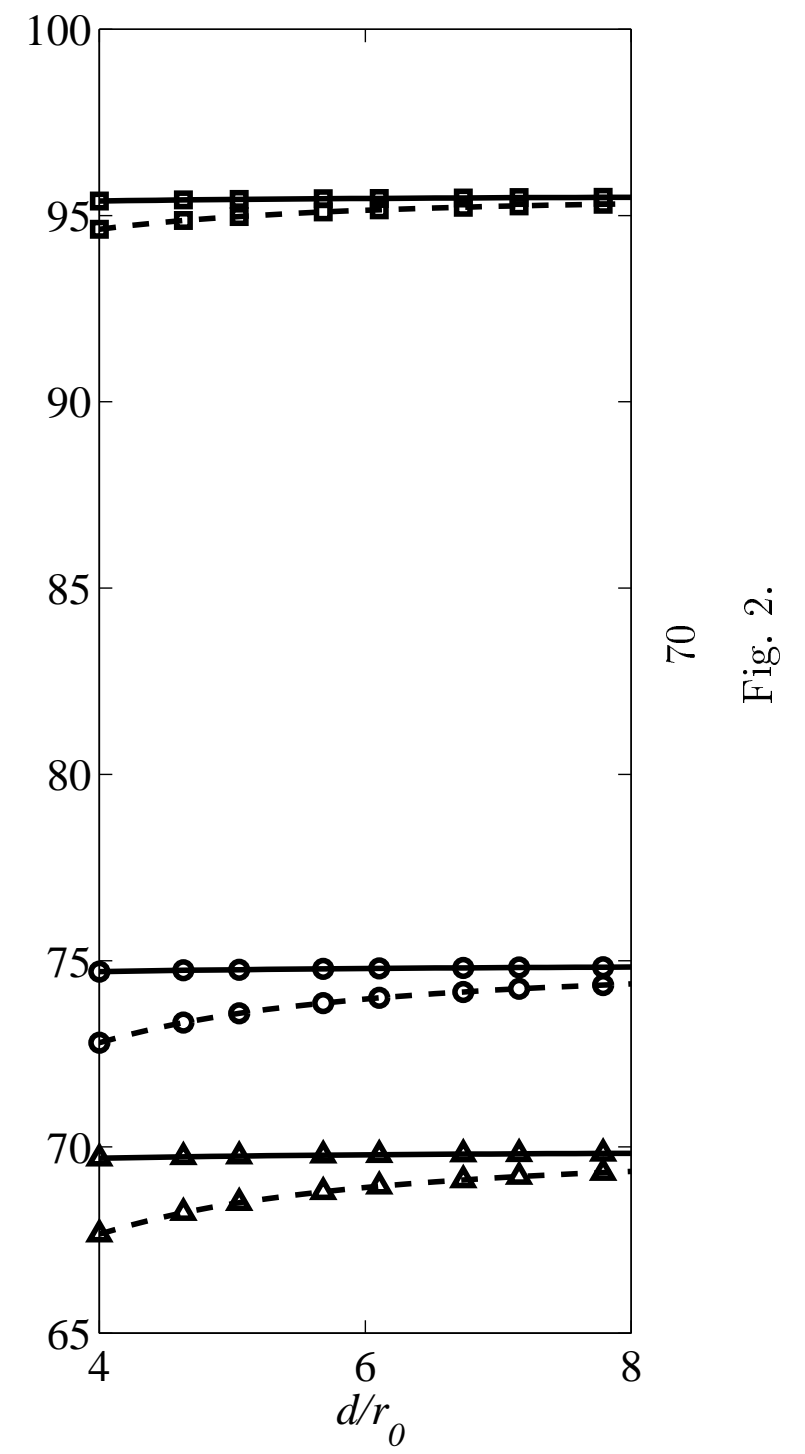


(a)

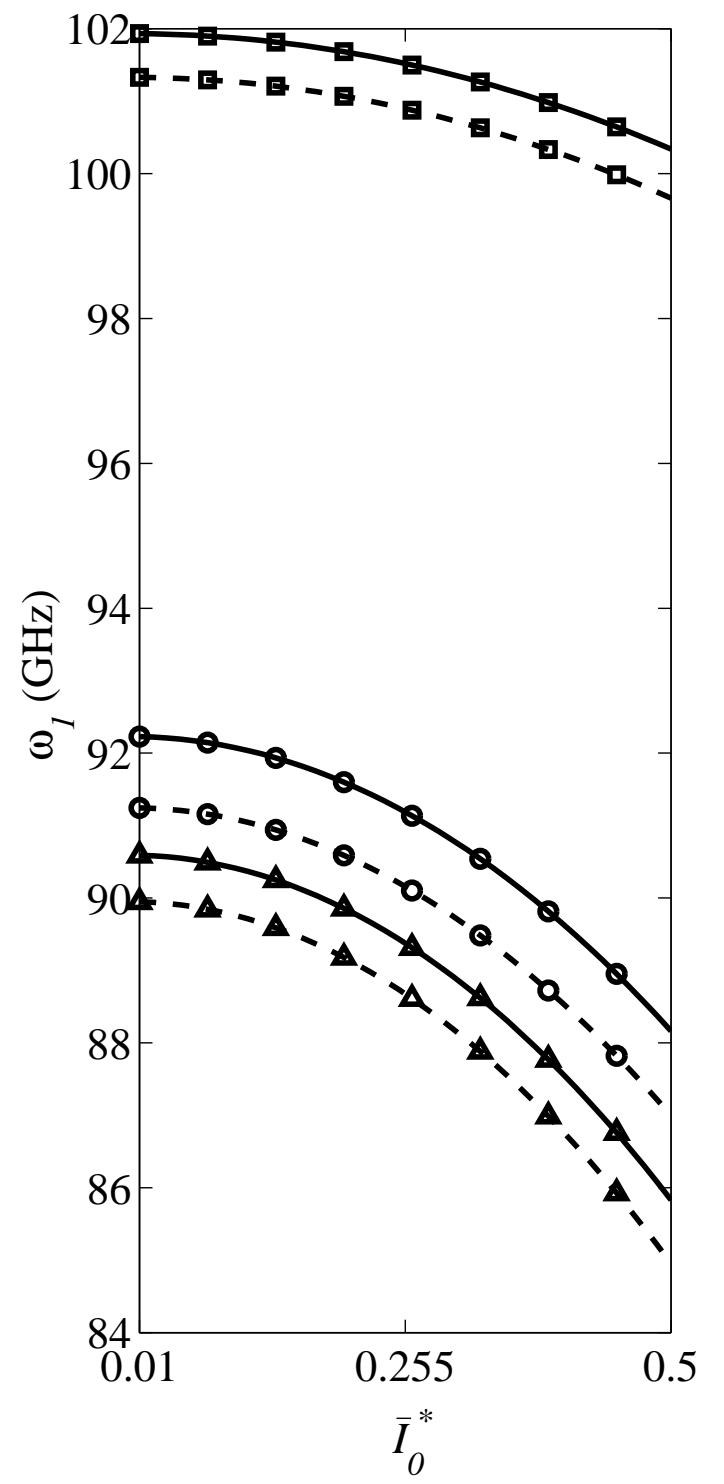

(b)

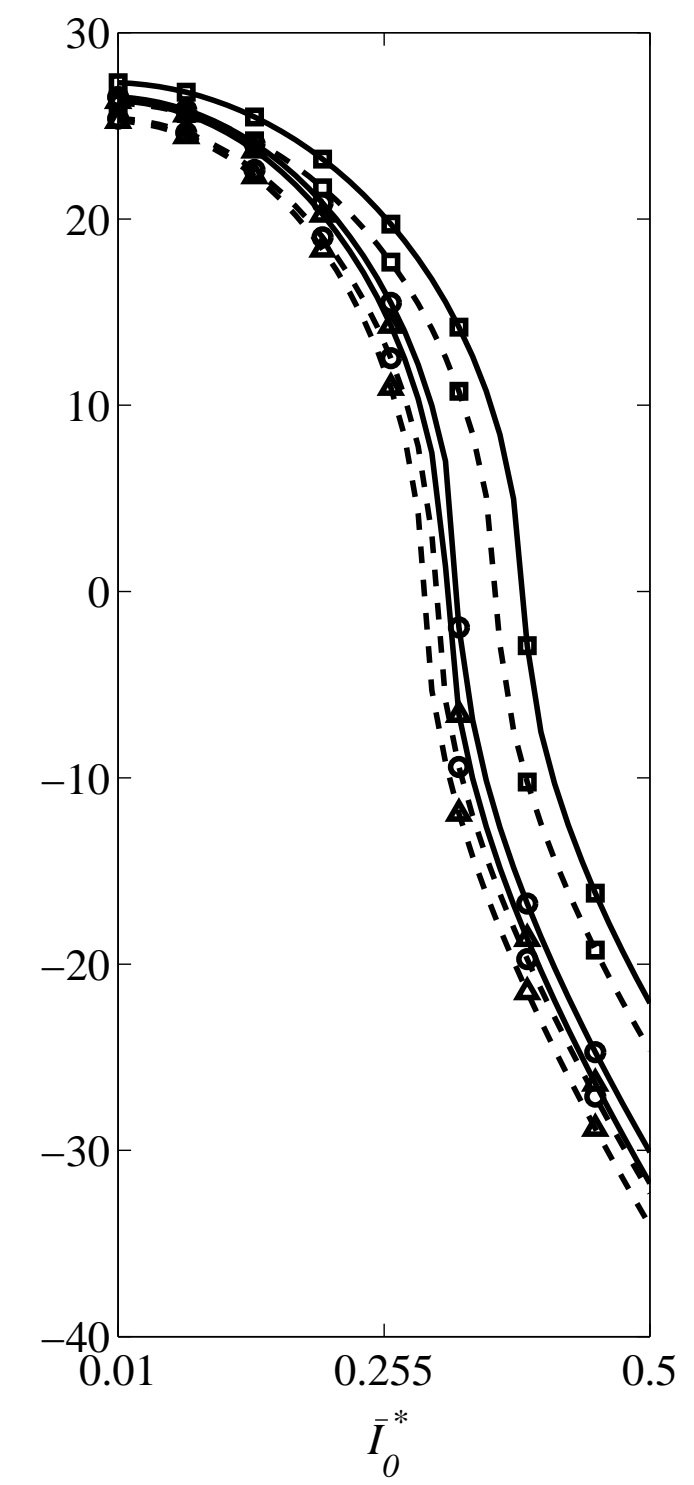

(c)

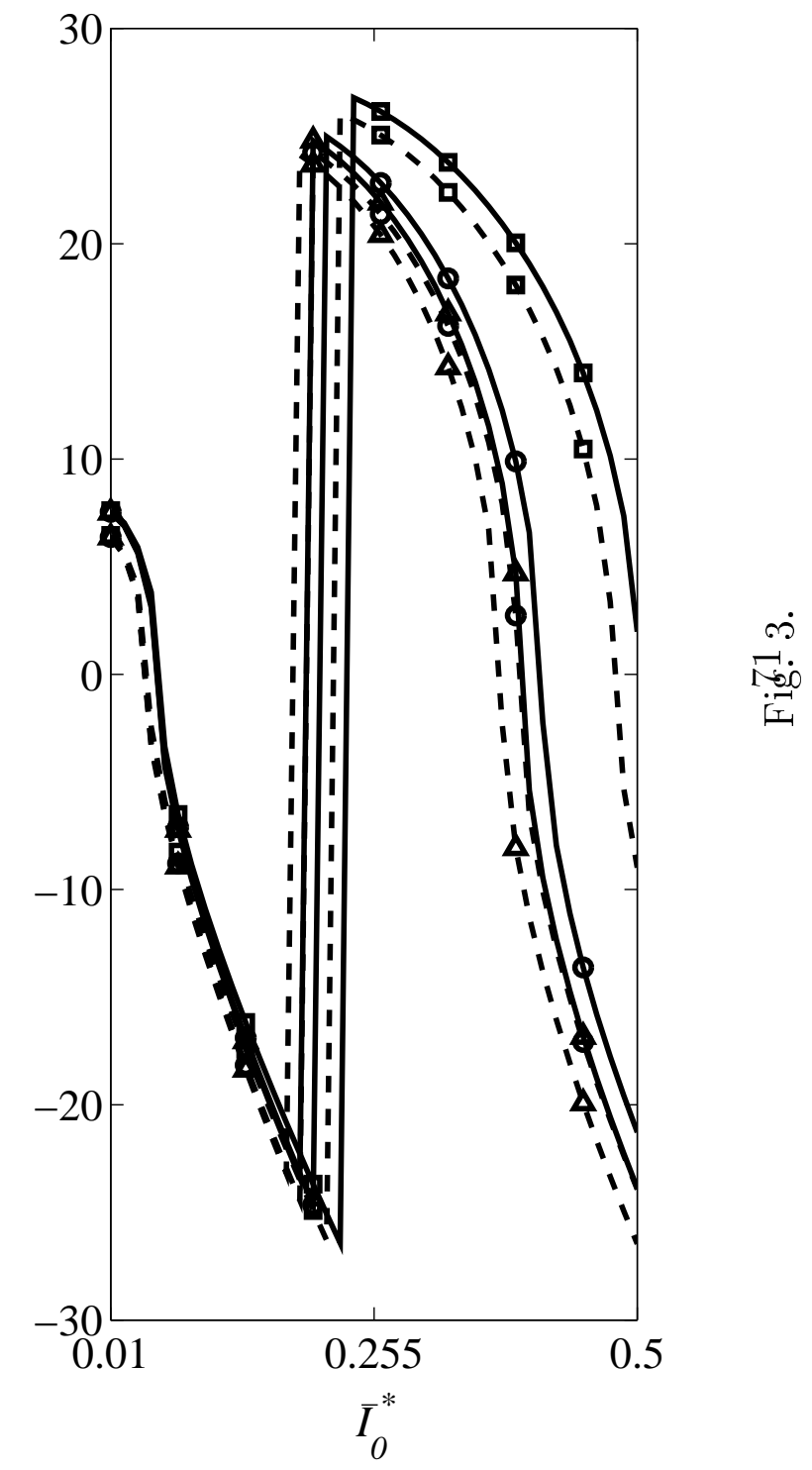


(a)

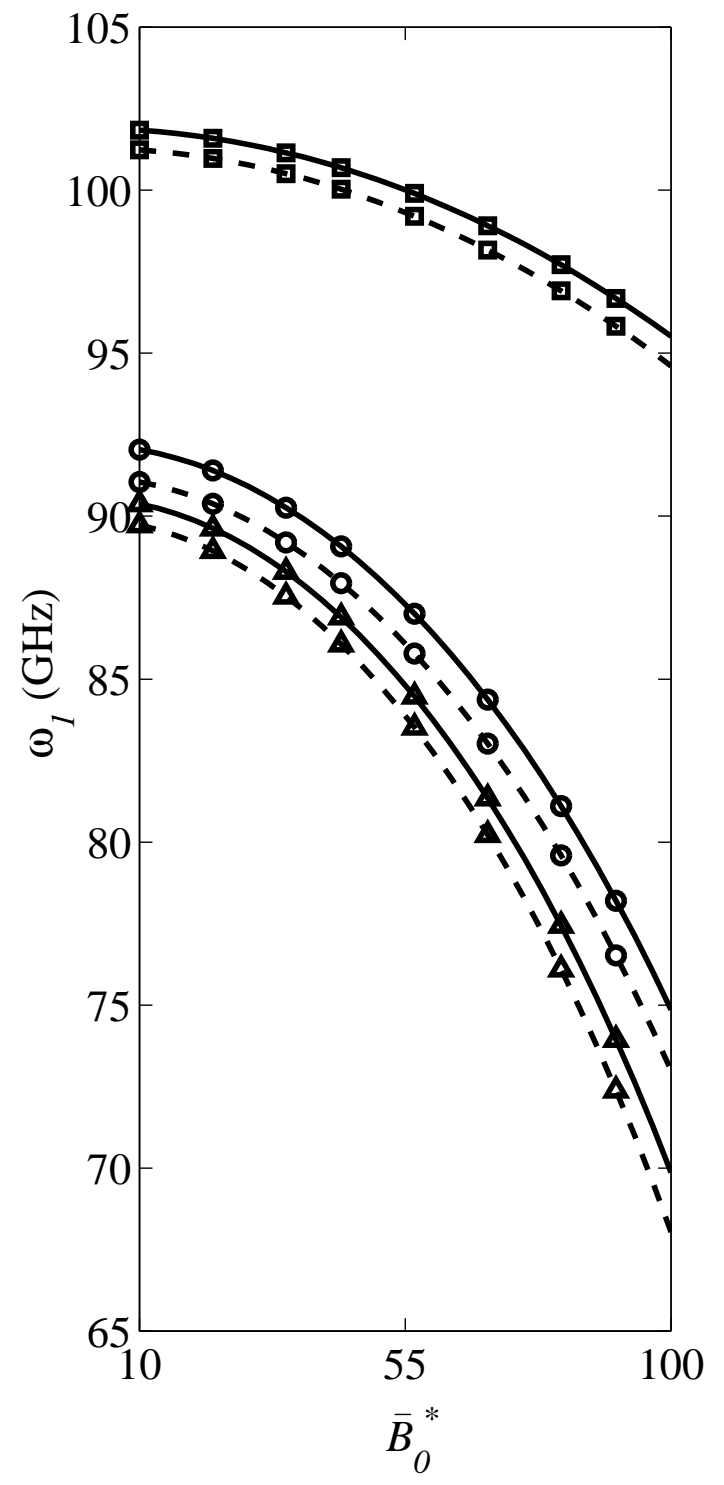

(b)

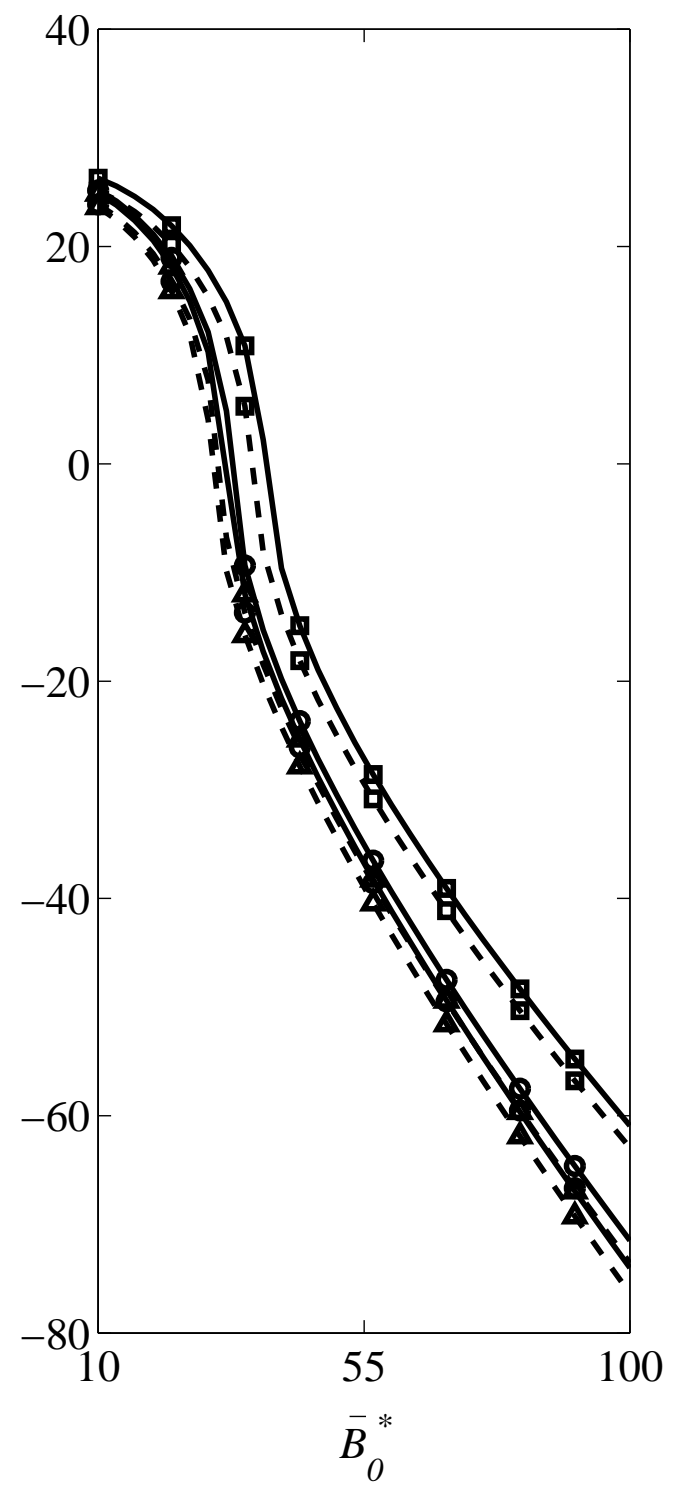

(c)

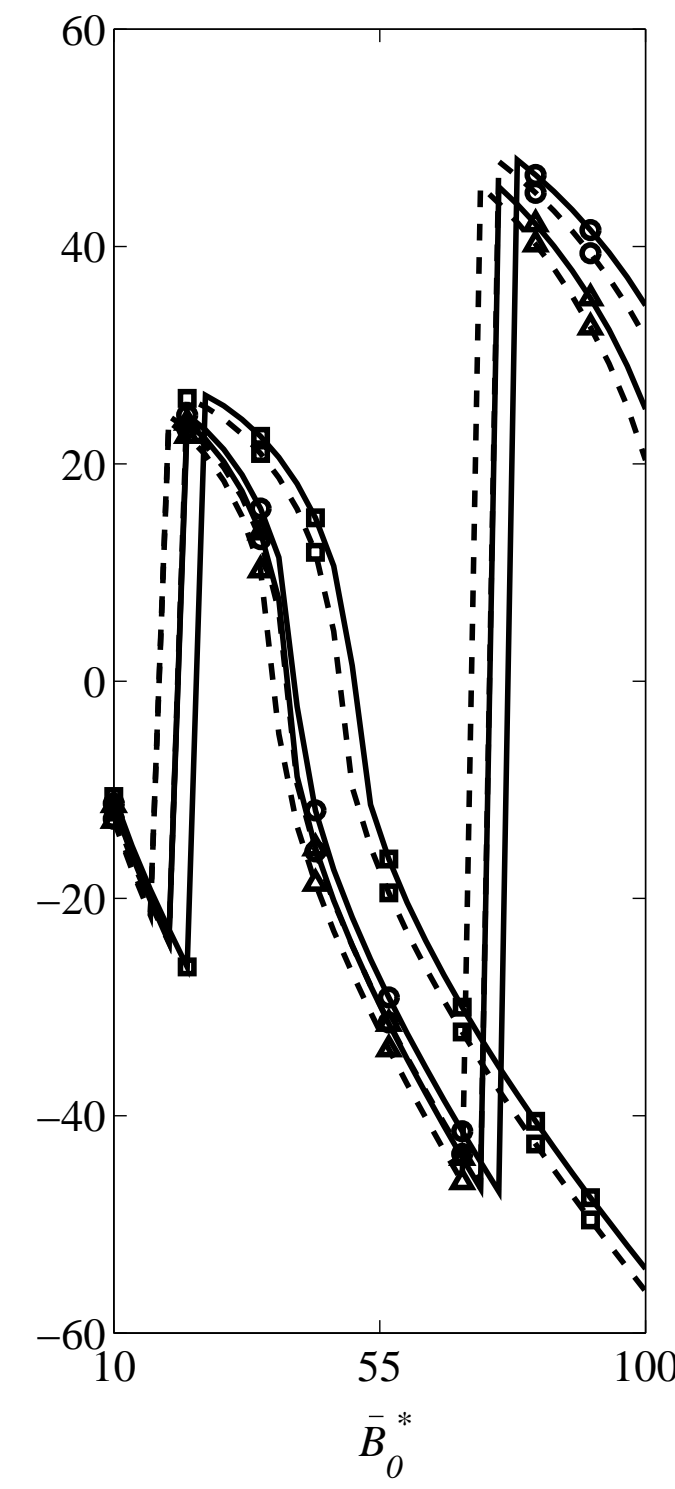


(a)

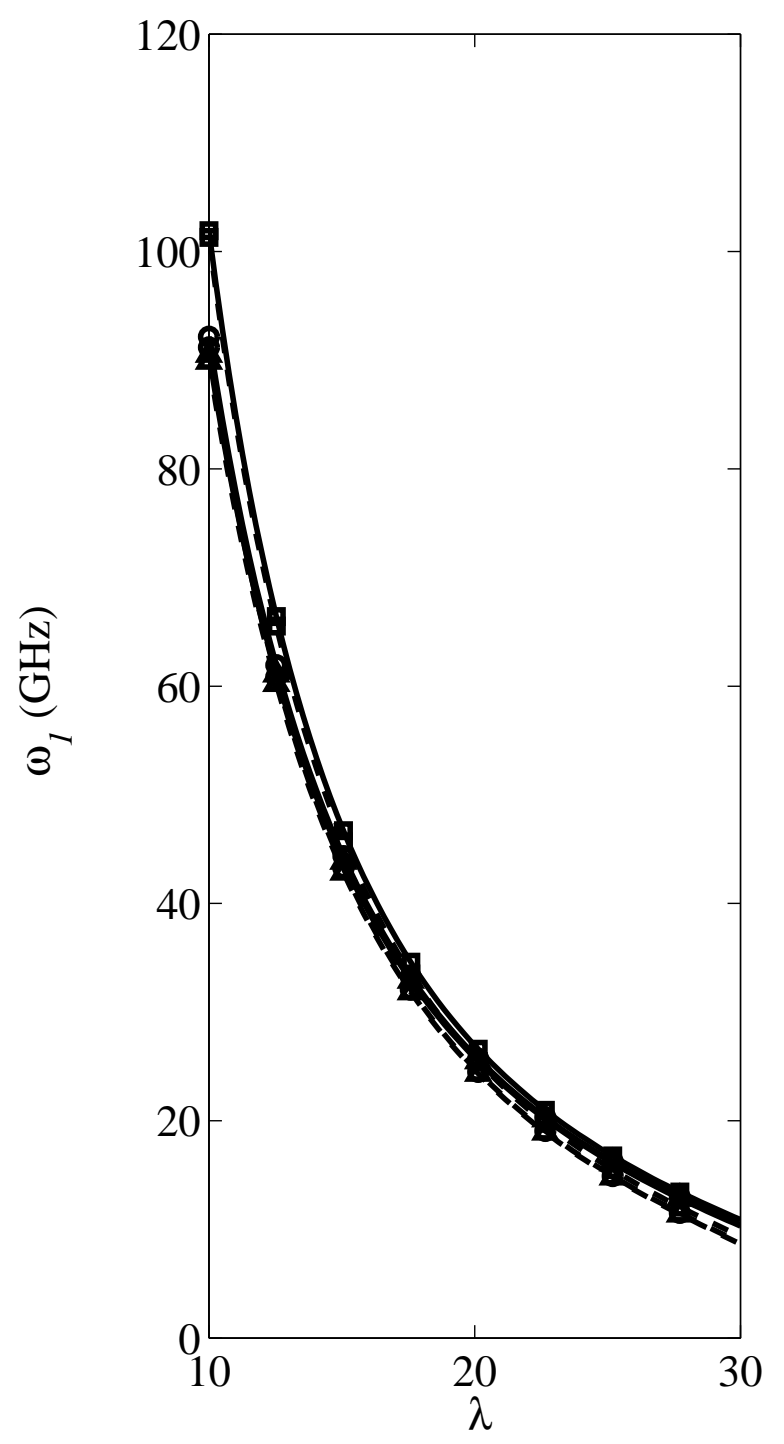

(b)

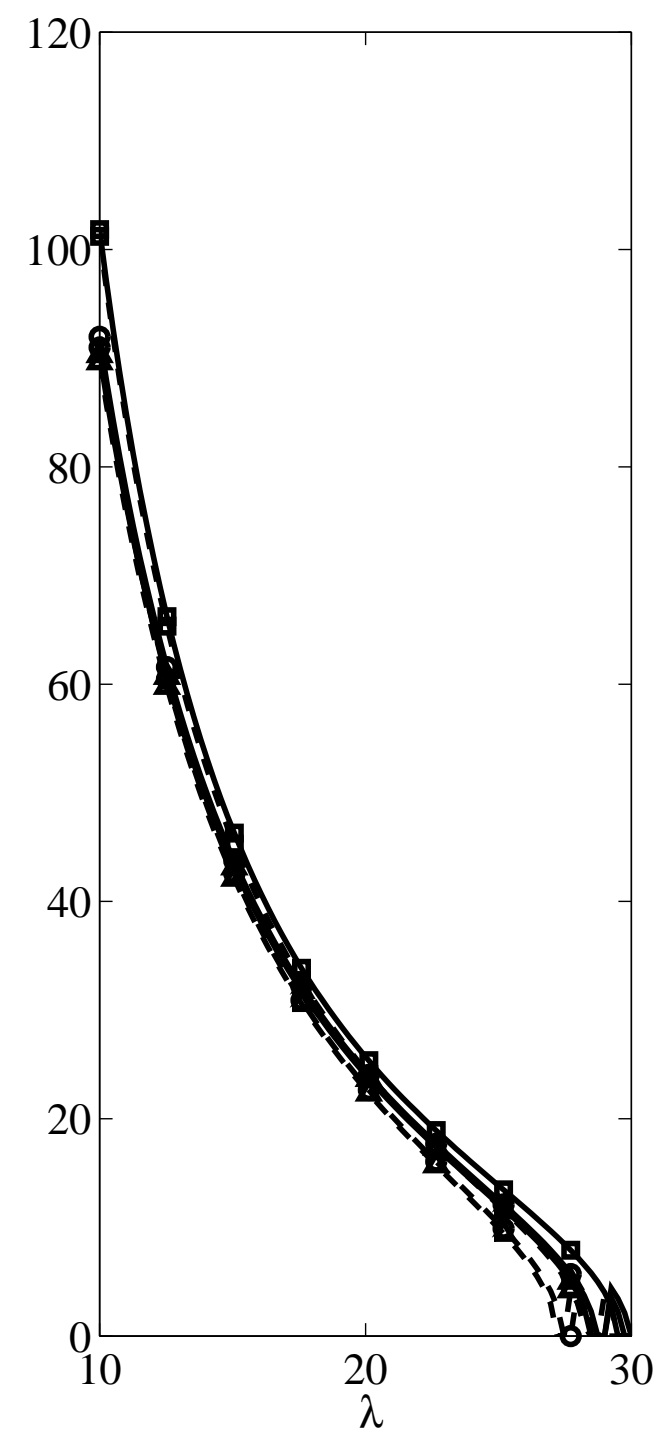

(c)

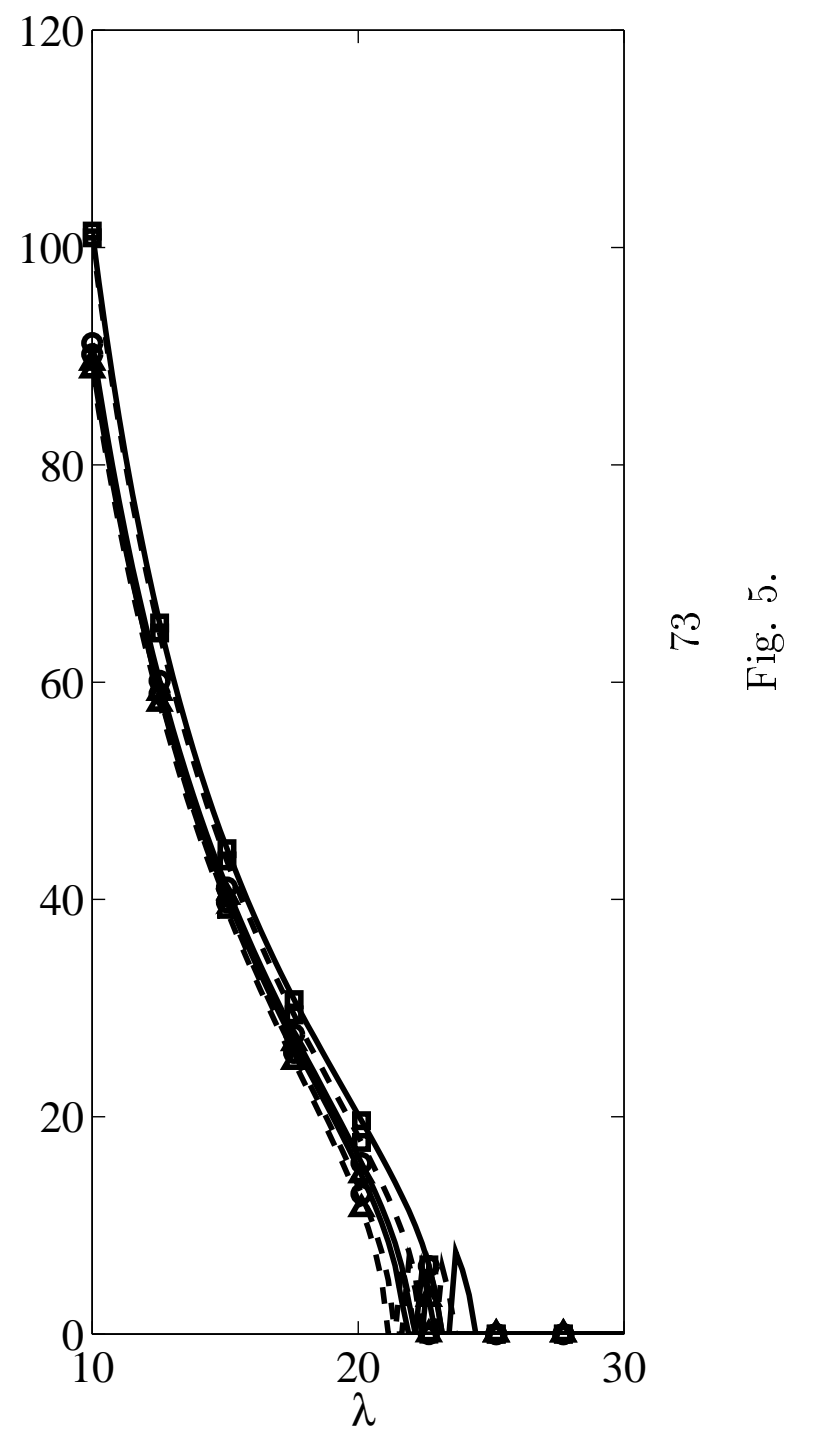


(a)

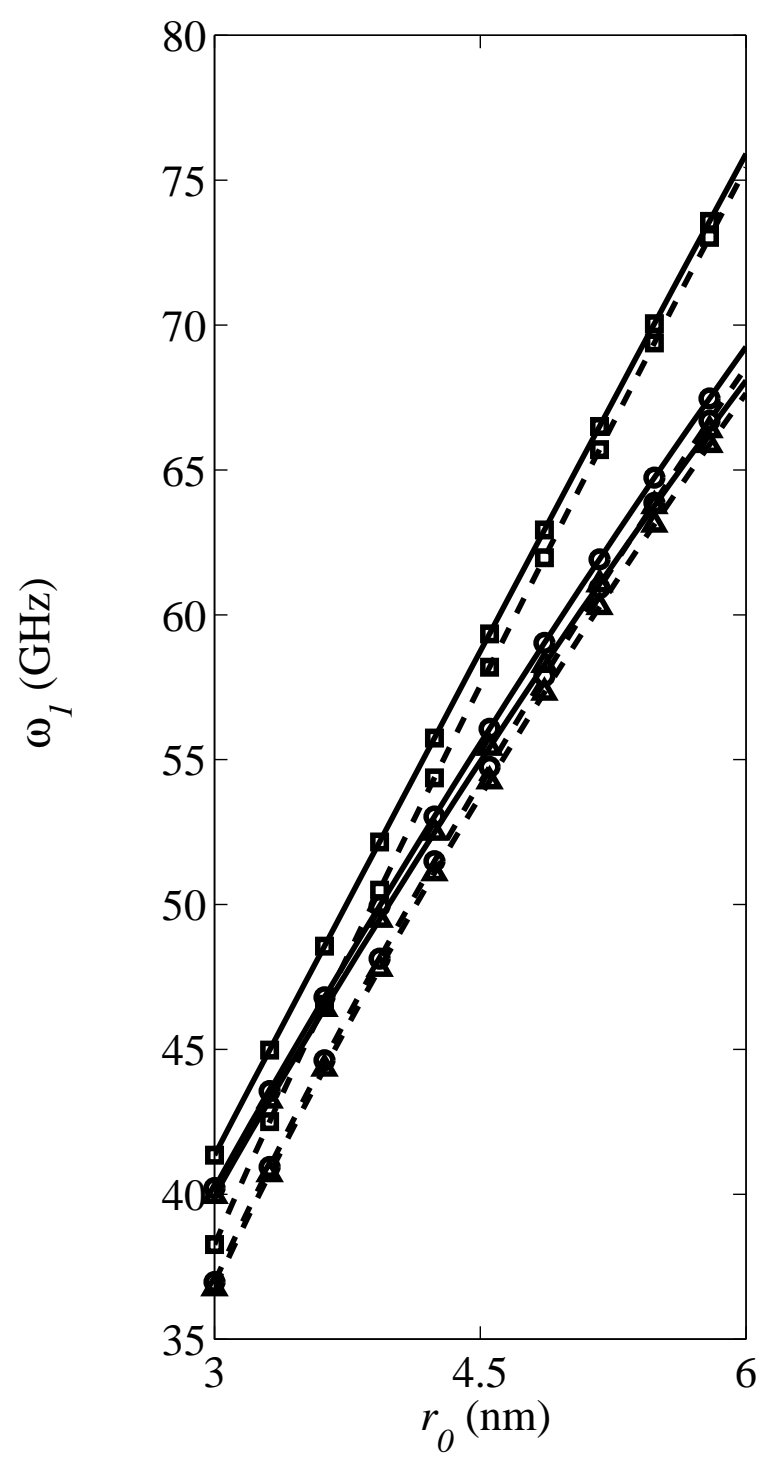

(b)

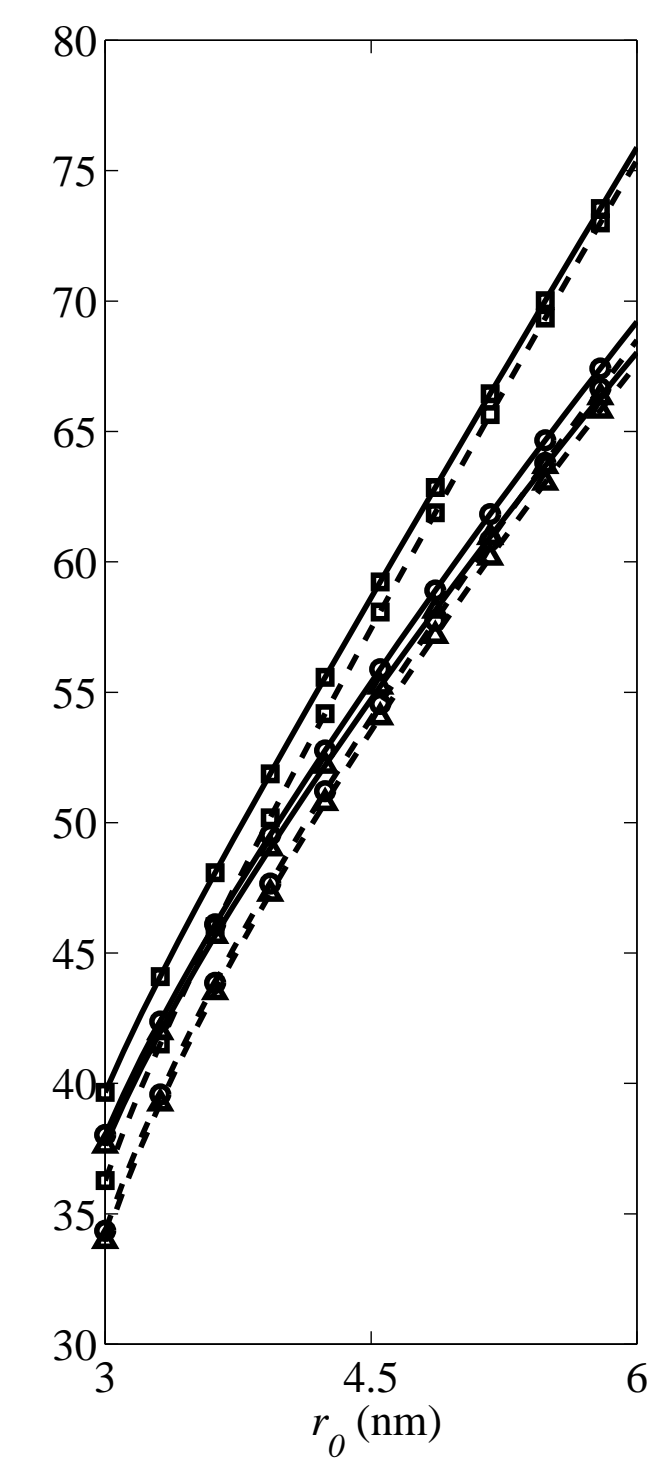

(c)

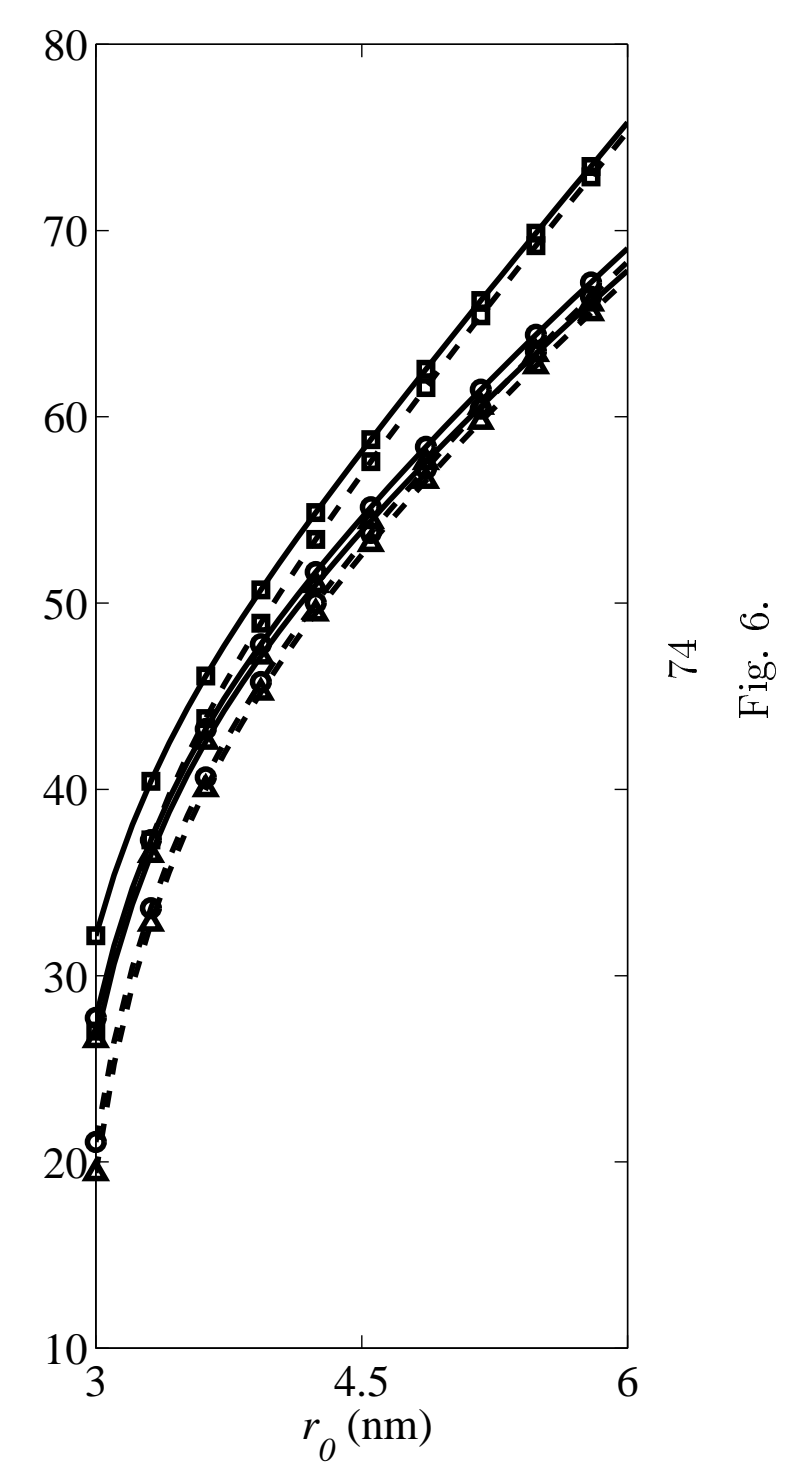

